\title{
ODOMETER ACTIONS OF THE HEISENBERG GROUP
}

\author{
Alexandre I. Danilenko And Mariusz LemanczyK
}

\begin{abstract}
Let $H_{3}(\mathbb{R})$ denote the 3-dimensional real Heisenberg group. Given a family of lattices $\Gamma_{1} \supset \Gamma_{2} \supset \cdots$ in it, let $T$ stand for the associated uniquely ergodic $H_{3}(\mathbb{R})$-odometer, i.e. the inverse limit of the $H_{3}(\mathbb{R})$-actions by rotations on the homogeneous spaces $H_{3}(\mathbb{R}) / \Gamma_{j}, j \in \mathbb{N}$. The decomposition of the underlying Koopman unitary representation of $H_{3}(\mathbb{R})$ into a countable direct sum of irreducible components is explicitly described. The ergodic 2-fold self-joinings of $T$ are found. It is shown that in general, the $H_{3}(\mathbb{R})$-odometers are neither isospectral nor spectrally determined.
\end{abstract}

\section{INTRODUCTION}

Let $T=\left(T_{g}\right)_{g \in G}$ be an ergodic measure preserving action of a locally compact second countable group $G$ on a standard probability space $(X, \mathfrak{B}, \mu)$. Denote by $U_{T}=\left(U_{T}(g)\right)_{g \in G}$ the associated Koopman unitary representation of $G$ in $L^{2}(X, \mu)$ :

$$
U_{T}(g) f:=f \circ T_{g}^{-1}, \quad f \in L^{2}(X, \mu) .
$$

Suppose first that $G$ is Abelian. If $U_{T}$ is a direct countable sum of 1-dimensional unitary sub-representations (generated by the $U_{T}$-eigenfunctions) then $T$ is said to have a pure point spectrum. In 1932, J. von Neumann [Ne] developed a theory of such actions in the case $G=\mathbb{R}$. We highlight three main aspects of this theory:

(A1) isospectrality: two ergodic flows with pure point spectrum are isomorphic if and only if the associated Koopman unitary representations are unitarily equivalent,

(A2) classification by simple algebraic invariants: the ergodic flows with pure point spectrum considered up to isomorphism are in one-to-one correspondence with the countable subgroups in $\widehat{\mathbb{R}}$ which is the dual of $\mathbb{R}$,

(A3) structure: if an ergodic flow has pure point spectrum then it is isomorphic to a flow by rotations on a compact metric Abelian group endowed with the Haar measure.

We also draw attention to a special subclass - apparently the simplest one - of flows with pure point rational spectrum. They are precisely those flows which admit a structure of inverse limit of transitive flows.

The results analogous to (A1)-(A3) hold for the general Abelian $G$ with similar proofs. The non-Abelian case - considered in this paper - is more intricate. G. Mackey in [Ma3] extended the concept of pure point spectrum to actions of

The second named author was supported in part by Narodowe Centrum Nauki grant DEC2011/03/B/ST1/00407. 
non-Abelian groups in the following way: $T$ has a pure point spectrum if $U_{T}$ is a direct sum of countably many finite dimensional unitary representations of $G$. He established a structure for these actions: an ergodic action $T$ has pure point spectrum if and only if it is isomorphic to a $G$-action by rotations on a homogeneous space of a compact group. However, in general, the $G$-actions with pure point spectrum are not isospectral even in the case of finite $G$. G. Mackey refers to [To] for a counterexample. (See also a discussion in [Le-We, Section 6].) Hence no classification for them is obtained.

In the present paper we consider the case where $G$ is the 3 -dimensional real Heisenberg group $H_{3}(\mathbb{R})$ which is apparently the 'simplest' non-Abelian nilpotent connected Lie group. Moreover, we single out a special class of actions of $H_{3}(\mathbb{R})$ which we call odometers. They are inverse limits of transitive $H_{3}(\mathbb{R})$-actions on homogeneous spaces by lattices in $H_{3}(\mathbb{R})$. In this connection, we note that for discrete finitely generated groups $G$, the $G$-odometers were considered by M. Cortez and $\mathrm{S}$. Petit in $[\mathrm{CoPe}]$ in the context of topological dynamics. We define $G$-odometers for arbitrary locally compact second countable groups $G$ and study them as measure preserving dynamical systems. Thus, by construction, the $H_{3}(\mathbb{R})$-odometers are counterparts of the $\mathbb{Z}$-actions with pure point rational spectrum. However, by taking into account definitions, the Heisenberg odometers are neither actions with pure point spectrum nor weakly mixing (the Cartesian square of a Heisenberg odometer is not ergodic).

Our purpose here is to investigate whether von Neumann's theory of flows with pure point spectrum extends (or partially extends) to the Heisenberg odometers.

We compute explicitly the spectrum of a Heisenberg odometer $T$, i.e. we describe the decomposition of $U_{T}$ into the direct countable sum of its irreducible components and we calculate their multiplicities in terms of the underlying sequence of lattices in $H_{3}(\mathbb{R})$ (Theorem 5.2). These components consist of two families: 1-dimensional unitary representations which occur in $U_{T}$ with multiplicity 1 and infinitely dimensional unitary representations. In the non-degenerate case (i.e. where the underlying $\mathbb{R}^{2}$-odometer is not transitive) every infinitely dimensional irreducible unitary representation that occurs in $U_{T}$ has there infinite multiplicity. To prove this result we use Kirillov's orbit method [Ki] and Howe-Richardson spectral multiplicity formula [Ho], [Ri]. The 1-dimensional family is parameterized by a so-called off-rational ${ }^{1}$ subgroup in $\mathbb{R}^{2}$ and the infinite dimensional family is parameterized by an off-rational subgroup in $\mathbb{R}$. Besides, there is a certain relation between these two subgroups. We prove that conversely, given two off-rational subgroups in $\mathbb{R}^{2}$ and $\mathbb{R}$ connected with this relation, there is a Heisenberg odometer whose Koopman representation is determined by these subgroups (Proposition 5.5). We show by example that the $H_{3}(\mathbb{R})$-odometers are not isospectral (Example 5.11).

Furthermore, we explicitly describe all ergodic 2-fold self-joinings of the Heisenberg odometers in Theorem 6.5. We provide an example of an ergodic 2-fold selfjoining of a transitive Heisenberg odometer which is neither transitive nor "odometric" at all (Example 6.4). We note that this is in marked contrast to the properties of locally compact transitive systems with pure point spectrum: each ergodic 2fold self-joining of such a system is transitive itself (and hence has a pure point spectrum).

Since our definition of the Heisenberg odometers is "structural", it seems natural

\footnotetext{
${ }^{1}$ See Definition 5.3.
} 
to investigate whether there is an equivalent spectral definition (as in the Abelian case). Thus we come to the following problem.

(A4) Spectral determinacy: if an $H_{3}(\mathbb{R})$-action is spectrally equivalent to a Heisenberg odometer, is it isomorphic to a Heisenberg odometer?

Answering (A3), we prove the spectral determinacy of the subclass of transitive Heisenberg odometers and, more generally, the subclass of Heisenberg odometers with transitive underlying $\mathbb{R}^{2}$-odometers (Theorem 7.1 ). However, the entire class of Heisenberg odometers is not spectrally determined. Nevertheless, we show that if the maximal spectral type of an $H_{3}(\mathbb{R})$-action $R$ coincides with the maximal spectral type of an $H_{3}(\mathbb{R})$-odometer then $R$ has the structure of a compact skew product extension of an $H_{3}(\mathbb{R})$-action with pure point spectrum. We obtain a criterion for when $R$ is conjugate to a $H_{3}(\mathbb{R})$-odometer in terms of the corresponding "extending" cocycle (Theorem 7.7). Using this criterion plus the orbit theory of amenable group actions we construct an example of a probability preserving $H_{3}(\mathbb{R})$ system which generates the same (up to the unitary equivalence) Koopman unitary representation as an $H_{3}(\mathbb{R})$-odometer but which is not isomorphic to any Heisenberg odometer (Example 7.11).

We also consider $H_{3}(\mathbb{Z})$-odometers and compare their properties with the properties of $H_{3}(\mathbb{R})$-odometers. In this connection it is interesting to note that $H_{3}(\mathbb{Z})$ is not of type $I$ and hence neither the unitary dual $\widehat{H_{3}(\mathbb{Z})}$ is a standard Borel space nor the spectral theorem (i.e. a unique decomposition of an arbitrary unitary representation of $\mathrm{H}_{3}(\mathbb{Z})$ into a direct integral of irreducible representations) holds. However for the class of $H_{3}(\mathbb{Z})$-odometers the situation is different. Every such odometer has a pure point spectrum in the sense of [Ma3] and the decomposition of the corresponding Koopman unitary representations of $H_{3}(\mathbb{Z})$ into irreducible components is well defined. Such decompositions for a subclass of normal free $H_{3}(\mathbb{Z})$-odometers were explicitly computed in $[\mathrm{Li}-\mathrm{Ug}]$. We note that the normal $H_{3}(\mathbb{Z})$-odometers are isospectral.

The outline of the paper is as follows. In Section 1 we discuss basic properties of $H_{3}(\mathbb{R})$, compute the group of its automorphisms and classify the lattices in $H_{3}(\mathbb{R})$. The odometer actions of locally compact groups are introduced in Section 2. Some general structural properties and the freeness of odometers are studied there. In Section 3 a convenient criterion for the freeness of Heisenberg odometers is found. In Section 4, for a transitive $H_{3}(\mathbb{R})$-odometer, the decomposition of the Koopman unitary representation $U_{T}$ into irreducible unitary representations of $H_{3}(\mathbb{R})$ is described. A similar decomposition for a general $H_{3}(\mathbb{R})$-odometer is obtained in Section 5. The corresponding measure of maximal spectral type sits on the union of two countable subgroups (in $\mathbb{R}^{2}$ and $\mathbb{R}$ ). All admissible pairs of such subgroups are described in Section 5. The fact that the $H_{3}(\mathbb{R})$-odometers are not isospectral is also proved in Section 5. Section 6 is devoted to a description of the ergodic 2-fold self-joinings of $H_{3}(\mathbb{R})$-odometers. In Section 7 we investigate spectral determinacy of the $H_{3}(\mathbb{R})$-odometers. In Section 8 we consider the $H_{3}(\mathbb{Z})$-odometers. Concluding remarks and open problems are discussed in the final Section 9.

Acknowledgements. We thank the referee for the useful remarks. 


\section{Preliminaries ON $H_{3}(\mathbb{R})$}

We recall that $H_{3}(\mathbb{R})$ consists of $3 \times 3$ upper triangular matrices of the form

$$
\left(\begin{array}{lll}
1 & a & c \\
0 & 1 & b \\
0 & 0 & 1
\end{array}\right)
$$

where $a, b, c$ are arbitrary reals. The Heisenberg group endowed with the natural topology is a connected, simply-connected nilpotent Lie group. We now let

$$
a(t):=\left(\begin{array}{ccc}
1 & t & 0 \\
0 & 1 & 0 \\
0 & 0 & 1
\end{array}\right), \quad b(t):=\left(\begin{array}{ccc}
1 & 0 & 0 \\
0 & 1 & t \\
0 & 0 & 1
\end{array}\right), \quad c(t):=\left(\begin{array}{ccc}
1 & 0 & t \\
0 & 1 & 0 \\
0 & 0 & 1
\end{array}\right) .
$$

Then $\{a(t) \mid t \in \mathbb{R}\},\{b(t) \mid t \in \mathbb{R}\}$ and $\{c(t) \mid t \in \mathbb{R}\}$ are three closed oneparameter subgroups in $H_{3}(\mathbb{R})$. The last is the center of $H_{3}(\mathbb{R})$. Every element $g$ of $H_{3}(\mathbb{R})$ can be written uniquely as the product $g=c\left(t_{3}\right) b\left(t_{2}\right) a\left(t_{1}\right)$ for some $t_{1}, t_{2}, t_{3} \in \mathbb{R}$. We also note that $\left[a\left(t_{1}\right), b\left(t_{2}\right)\right]:=a\left(t_{1}\right) b\left(t_{2}\right) a\left(t_{1}\right)^{-1} b\left(t_{2}\right)^{-1}=c\left(t_{1} t_{2}\right)$ and the commutator of $H_{3}(\mathbb{R})$ equals the center of $H_{3}(\mathbb{R})$.

The subgroups $H_{2, a}:=\left\{a\left(t_{1}\right) c\left(t_{3}\right) \mid t_{1}, t_{3} \in \mathbb{R}\right\}$ and $H_{2, b}:=\left\{b\left(t_{2}\right) c\left(t_{3}\right) \mid t_{2}, t_{3} \in\right.$ $\mathbb{R}\}$ are both Abelian, normal and closed in $H_{3}(\mathbb{R})$. The corresponding group extensions

$$
\begin{aligned}
& 0 \rightarrow H_{2, a} \rightarrow H_{3}(\mathbb{R}) \rightarrow H_{3}(\mathbb{R}) / H_{2, a} \rightarrow 0 \text { and } \\
& 0 \rightarrow H_{2, b} \rightarrow H_{3}(\mathbb{R}) \rightarrow H_{3}(\mathbb{R}) / H_{2, b} \rightarrow 0
\end{aligned}
$$

both split. This implies that $H_{3}(\mathbb{R})$ is isomorphic to the semidirect product $\mathbb{R}^{2} \rtimes_{B} \mathbb{R}$, where the homomorphism $B: \mathbb{R} \rightarrow \mathrm{GL}_{2}(\mathbb{R})$ is given by $B(t):=\left(\begin{array}{ll}1 & t \\ 0 & 1\end{array}\right), t \in \mathbb{R}$. The subgroups $H_{2, a}$ and $H_{2, b}$ are automorphic in $H_{3}(\mathbb{R})$, i.e. there is an isomorphism $\theta$ of $H_{3}(\mathbb{R})$ with $\theta\left(H_{2, a}\right)=H_{2, b}$. We define $\theta$ by setting $\theta(a(t)):=b(t)$, $\phi(b(t)):=a(t)$ and $\theta(c(t)):=c(-t)$ for all $t \in \mathbb{R}$. To put it another way,

$$
\theta\left(\begin{array}{ccc}
1 & a & c \\
0 & 1 & b \\
0 & 0 & 1
\end{array}\right)=\left(\begin{array}{ccc}
1 & b & a b-c \\
0 & 1 & a \\
0 & 0 & 1
\end{array}\right) .
$$

We call $\theta$ the flip in $H_{3}(\mathbb{R})$. We note that $\theta^{2}=$ id.

The set of unitarily equivalent classes of irreducible (weakly continuous) representations of $H_{3}(\mathbb{R})$ is called the unitary dual of $H_{3}(\mathbb{R})$. It is denoted by $\widehat{H_{3}(\mathbb{R})}$. The irreducible unitary representations of $H_{3}(\mathbb{R})$ are well known. They consist (up to unitary equivalence) of a family of 1-dimensional representations $\pi_{\alpha, \beta}, \alpha, \beta \in \mathbb{R}$, and a family of infinite dimensional representations $\pi_{\gamma}, \gamma \in \mathbb{R} \backslash\{0\}$, as follows [Ki]:

$$
\begin{aligned}
\pi_{\alpha, \beta}\left(c\left(t_{3}\right) b\left(t_{2}\right) a\left(t_{1}\right)\right) & :=e^{2 \pi i\left(\alpha t_{1}+\beta t_{2}\right)} \quad \text { and } \\
\left(\pi_{\gamma}\left(c\left(t_{3}\right) b\left(t_{2}\right) a\left(t_{1}\right)\right) f\right)(x) & :=e^{2 \pi i \gamma\left(t_{3}+t_{2} x\right)} f\left(x+t_{1}\right), \quad f \in L^{2}\left(\mathbb{R}, \lambda_{\mathbb{R}}\right) .
\end{aligned}
$$

Thus we can identify $\widehat{H_{3}(\mathbb{R})}$ with the disjoint union $\mathbb{R}^{2} \sqcup \mathbb{R}^{*}$. We recall that there is a natural Borel structure on the unitary dual of each locally compact second 
countable group [Ma2]. In the case of the Heisenberg group this Borel $\sigma$-algebra coincides with the standard $\sigma$-algebra of Borel subsets in $\mathbb{R}^{2} \sqcup \mathbb{R}^{*}$.

Given an arbitrary unitary representation $U=(U(g))_{g \in H_{3}(\mathbb{R})}$ of $H_{3}(\mathbb{R})$ in a separable Hilbert space $\mathcal{H}$, there are a measure $\sigma_{U}$ on $\widehat{H_{3}(\mathbb{R})}$ (i.e. two measures $\sigma_{U}^{1,2}$ on $\mathbb{R}^{2}$ and $\sigma_{U}^{3}$ on $\mathbb{R}^{*}$ ) and a map $l_{U}: \widehat{H_{3}(\mathbb{R})} \rightarrow \mathbb{N} \cup\{\infty\}$ (i.e. two maps $l_{U}^{1,2}: \mathbb{R}^{2} \ni(x, y) \mapsto l_{U}^{1,2}(x, y) \in \mathbb{N} \cup\{\infty\}$ and $\left.l_{U}^{3}: \mathbb{R}^{*} \ni z \mapsto l_{U}^{3}(z) \in \mathbb{N} \cup\{\infty\}\right)$ such that the following decompositions hold (up to the unitary equivalence):

$$
\begin{aligned}
\mathcal{H} & =\int_{\mathbb{R}^{2}}^{\oplus} \bigoplus_{j=1}^{l_{U}^{1,2}(\alpha, \beta)} \mathbb{C} d \sigma_{U}^{1,2}(\alpha, \beta) \oplus \int_{\mathbb{R}^{*}}^{\oplus} \bigoplus_{j=1}^{l_{U}^{3}(\gamma)} L^{2}\left(\mathbb{R}, \lambda_{\mathbb{R}}\right) d \sigma_{U}^{3}(\gamma) \quad \text { and } \\
U(g) & =\int_{\mathbb{R}^{2}}^{\oplus} \bigoplus_{j=1}^{l_{U}^{1,2}(\alpha, \beta)} \pi_{\alpha, \beta}(g) d \sigma_{U}^{1,2}(\alpha, \beta) \oplus \int_{\mathbb{R}^{*}}^{\oplus} \bigoplus_{j=1}^{l_{U}^{3}(\gamma)} \pi_{\gamma}(g) d \sigma_{U}^{3}(\gamma) .
\end{aligned}
$$

The equivalence class of $\sigma_{U}$ is called the maximal spectral type of $U$. The map $l_{U}$ is called the multiplicity function of $U$. The essential range of $l_{U}$ is called the set of spectral multiplicities of $U$. The maximal spectral type and the multiplicity function of $U\left(\sigma_{U}\right.$-mod 0$)$ are both determined uniquely by the unitary equivalence class of $U$.

Below, in this section we:

(a) describe explicitly the group of all continuous automorphisms of the Heisenberg group and

(b) classify the lattices in $H_{3}(\mathbb{R})$ up to automorphism of $H_{3}(\mathbb{R})$.

Although these results are not new, we think it is easier to reprove them here than to find them in the literature and "adjust" to our notation.

From now on we denote by $p$ the natural projection

$$
H_{3}(\mathbb{R}) \ni g \mapsto p(g):=\left(t_{1}, t_{2}\right) \in \mathbb{R}^{2}
$$

whenever $g=c\left(t_{3}\right) b\left(t_{2}\right) a\left(t_{1}\right)$.

Let $\widetilde{\theta}$ be an automorphism of $H_{3}(\mathbb{R})$. Of course, $\widetilde{\theta}$ preserves the center of $H_{3}(\mathbb{R})$. Hence there exists $\tau \in \mathbb{R}^{*}$ such that

$$
\tilde{\theta}(c(t))=c(\tau t), \quad t \in \mathbb{R} .
$$

Since the center of $H_{3}(\mathbb{R})$ is the kernel of $p, \widetilde{\theta}$ passes through $p$, i.e. there is a matrix $A=\left(\begin{array}{ll}\xi_{1} & \eta_{1} \\ \xi_{2} & \eta_{2}\end{array}\right) \in G L_{2}(\mathbb{R})$ such that $p \circ \widetilde{\theta}=A \circ p$. Therefore we can write $\widetilde{\theta}(a(t))$ as

$$
\widetilde{\theta}(a(t))=c(f(t)) b\left(\xi_{2} t\right) a\left(\xi_{1} t\right), \quad t \in \mathbb{R},
$$

for some continuous function $f: \mathbb{R} \rightarrow \mathbb{R}$. Since $\widetilde{\theta}\left(a\left(t_{1}+t_{2}\right)\right)=\widetilde{\theta}\left(a\left(t_{1}\right)\right) \widetilde{\theta}\left(a\left(t_{2}\right)\right)$, it follows that

$$
f\left(t_{1}+t_{2}\right)=f\left(t_{1}\right)+f\left(t_{2}\right)+\xi_{1} \xi_{2} t_{1} t_{2}
$$

for all $t_{1}, t_{2} \in \mathbb{R}$. Therefore $f(t)=\frac{\xi_{1} \xi_{2}}{2} t^{2}+\xi t$ for some real $\xi \in \mathbb{R}$. Thus,

$$
\widetilde{\theta}(a(t))=c\left(\frac{\xi_{1} \xi_{2}}{2} t^{2}+\xi t\right) b\left(\xi_{2} t\right) a\left(\xi_{1} t\right), \quad t \in \mathbb{R}
$$


In a similar way,

$$
\widetilde{\theta}(b(t))=c\left(\frac{\eta_{1} \eta_{2}}{2} t^{2}+\eta t\right) b\left(\eta_{2} t\right) a\left(\eta_{1} t\right), \quad t \in \mathbb{R}
$$

for some $\eta \in \mathbb{R}$. Since $\widetilde{\theta}$ is a homomorphism of $H_{3}(\mathbb{R})$, we have

$$
\widetilde{\theta}\left(a\left(t_{1}\right)\right) \widetilde{\theta}\left(b\left(t_{2}\right)\right)=\widetilde{\theta}\left(a\left(t_{1}\right) b\left(t_{2}\right)\right)=\widetilde{\theta}\left(b\left(t_{2}\right) a\left(t_{1}\right) c\left(t_{1} t_{2}\right)\right)=\widetilde{\theta}\left(b\left(t_{2}\right)\right) \widetilde{\theta}\left(a\left(t_{1}\right)\right) \widetilde{\theta}\left(c\left(t_{1} t_{2}\right)\right)
$$

for all $t_{1}, t_{2} \in \mathbb{R}$. Applying (1-2) and (1-3) and then (1-1), we obtain

$$
c\left(\xi_{1} \eta_{2} t_{1} t_{2}\right)=c\left(\eta_{1} \xi_{2} t_{1} t_{2}+\tau t_{1} t_{2}\right)
$$

which yields $\tau=\operatorname{det} A$. Thus, the three parameters $A, \xi, \eta$ determine $\widetilde{\theta}$ completely. Conversely, given $A \in G L_{2}(\mathbb{R})$ and $\xi, \eta \in \mathbb{R}$, the formulas (1-1)-(1-3) with $\tau=\operatorname{det} A$ determine completely an automorphism of $H_{3}(\mathbb{R})$. We will denote it by $\theta_{A, \xi, \eta}$.

Thus, we have proved the following proposition.

Proposition 1.1. $\operatorname{Aut}\left(H_{3}(\mathbb{R})\right)=\left\{\theta_{A, \xi, \eta} \mid A \in G L_{2}(\mathbb{R}), \xi, \eta \in \mathbb{R}\right\}$.

We also note that $N:=\left\{\theta_{I, \xi, \eta} \mid \xi, \eta \in \mathbb{R}\right\}$ is a normal subgroup in $\operatorname{Aut}\left(H_{3}(\mathbb{R})\right)$. It is isomorphic naturally to $\mathbb{R}^{2}$. In fact, $N$ is the subgroup of inner automorphisms of $H_{3}(\mathbb{R})$. We obtain a short exact sequence

$$
\{1\} \leftarrow G L_{2}(\mathbb{R}) \leftarrow \operatorname{Aut}\left(H_{3}(\mathbb{R})\right) \leftarrow N \leftarrow\{1\}
$$

Moreover, a direct calculation shows that the homomorphism

$$
G L_{2}(\mathbb{R}) \ni A \mapsto \theta_{A, 0,0} \in \operatorname{Aut}\left(H_{3}(\mathbb{R})\right)
$$

is a cross-section of the natural projection in (1-4).

Corollary 1.2. Aut $\left(H_{3}(\mathbb{R})\right)$ is isomorphic to the semidirect product $\mathbb{R}^{2} \rtimes G L_{2}(\mathbb{R})$, where the corresponding action of $G L_{2}(\mathbb{R})$ on $\mathbb{R}^{2}$ is given by $A v:=\operatorname{det} A \cdot\left(A^{*}\right)^{-1} v$.

We now describe the structure of lattices in the Heisenberg group and classify them up to group automorphism. Recall that a lattice is a discrete subgroup of finite covolume. In the case of a simply connected nilpotent Lie group, every lattice is cocompoct $[\mathrm{Ra}]$. Fix a lattice $\Gamma$ in $H_{3}(\mathbb{R})$. There is a real $\xi_{\Gamma}>0^{2}$ such that

$$
\Gamma \cap\{c(t) \mid t \in \mathbb{R}\}=\left\{c\left(m \xi_{\Gamma}\right) \mid m \in \mathbb{Z}\right\}
$$

The central extension

$$
\{0\} \leftarrow \mathbb{R}^{2} \stackrel{p}{\leftarrow} H_{3}(\mathbb{R}) \stackrel{c}{\leftarrow} \mathbb{R} \leftarrow\{0\}
$$

\footnotetext{
${ }^{2}$ If $\xi_{\Gamma}=0$ then the intersection of $\Gamma$ with the center of $H_{3}(\mathbb{R})$ is trivial. This yields that $\Gamma$ is Abelian and hence there is a line (i.e. a 1-dimensional subspace) $\mathcal{L}$ in $\mathbb{R}^{2}$ such that $\Gamma$ is contained in a normal closed subgroup $H:=\left\{c(t) b\left(t_{2}\right) a\left(t_{1}\right) \mid\left(t_{1}, t_{2}\right) \in \mathcal{L}, t \in \mathbb{R}\right\}$ of $H_{3}(\mathbb{R})$. Therefore the quotient group $H_{3}(\mathbb{R}) / H$ being a quotient space of $H_{3}(\mathbb{R}) / \Gamma$ is of finite Haar measure, i.e. compact. However, it is straightforward to verify that $H_{3}(\mathbb{R}) / H$ is isomorphic to $\mathbb{R}$, a contradiction.
} 
induces a short exact sequence

$$
\{0\} \longleftarrow p(\Gamma) \stackrel{p}{\longleftarrow} \Gamma \stackrel{c}{\longleftarrow} \xi_{\Gamma} \mathbb{Z} \longleftarrow\{0\}
$$

We note that $p(\Gamma)$ is a lattice in $\mathbb{R}^{2}{ }^{3}$ Therefore there is a matrix $A \in G L_{2}(\mathbb{R})$ such that $p\left(\theta_{A, 0,0}(\Gamma)\right)=A p(\Gamma)=\mathbb{Z}^{2}$. The commutator subgroup $[\Gamma, \Gamma]$ is of a finite index $k_{\Gamma}>0$ in $p^{-1}(0) \cap \Gamma$.

It is easy to see that $k_{\theta(\Gamma)}=k_{\Gamma}$ for each $\theta \in \operatorname{Aut}\left(H_{3}(\mathbb{R})\right)$. Let $\zeta$ and $\eta$ be the smallest non-negative reals such that $c(\zeta) b(0) a(1) \in \theta_{A, 0,0}(\Gamma)$ and $c(\eta) b(1) a(0) \in$ $\theta_{A, 0,0}(\Gamma)$. Then $\theta_{I,-\zeta,-\eta}(c(\zeta) a(1))=a(1)$ and $\theta_{I,-\zeta,-\eta}(c(\eta) a(1))=b(1)$. We let $\widetilde{\Gamma}:=\theta_{I,-\zeta,-\eta}\left(\theta_{A, 0,0}(\Gamma)\right)$. Then $a(1), b(1) \in \widetilde{\Gamma}$. Hence $[a(1), b(1)]=c(1) \in \widetilde{\Gamma}$. On the other hand, $[a(1), b(1)]=c\left(k_{\widetilde{\Gamma}} \xi_{\widetilde{\Gamma}}\right)=c\left(k_{\Gamma} \xi_{\widetilde{\Gamma}}\right)$. It follows that $\xi_{\widetilde{\Gamma}}=1 / k_{\Gamma}$. The elements $a(1), b(1)$ and $c\left(k_{\Gamma}^{-1}\right)$ generate the lattice $\widetilde{\Gamma}$. Thus we have proved the following proposition.

Proposition 1.3. Given a lattice $\Gamma$ in $H_{3}(\mathbb{R})$, there is an automorphism $\theta$ of $H_{3}(\mathbb{R})$ such that

$$
\theta(\Gamma)=\left\{\left(\begin{array}{ccc}
1 & l & \frac{n}{k_{\Gamma}} \\
0 & 1 & m \\
0 & 0 & 1
\end{array}\right) \mid l, m, n \in \mathbb{Z}\right\} .
$$

Hence two lattices $\Gamma_{1}$ and $\Gamma_{2}$ in $H_{3}(\mathbb{R})$ are automorphic if and only if $k_{\Gamma_{1}}=k_{\Gamma_{2}}$. Two lattices $\Gamma_{1}$ and $\Gamma_{2}$ in $H_{3}(\mathbb{R})$ are conjugate if and only if $k_{\Gamma_{1}}=k_{\Gamma_{2}}$ and $p\left(\Gamma_{1}\right)=$ $p\left(\Gamma_{2}\right)$.

Corollary 1.4. If an element $g \in H_{3}(\mathbb{R})$ commutes with each element of a lattice $\Gamma$ in $H_{3}(\mathbb{R})$ then $g$ belongs to the center of $H_{3}(\mathbb{R})$.

The following lemma will be used in the proof of Theorem 5.7 which is one of the main results of this paper.

Lemma 1.5. Let $\Gamma$ and $\Lambda$ be two lattices in $H_{3}(\mathbb{R})$ such that $\Lambda \subset \Gamma$. Let $p(\Gamma)=$ $A\left(\mathbb{Z}^{2}\right)$ for a matrix $A \in G L_{2}(\mathbb{R})$. Then for each positive real $\xi$ such that $\xi / \xi_{\Gamma} \in \mathbb{N}$, $\xi_{\Lambda} / \xi \in \mathbb{N}$ and $|\operatorname{det} A| / \xi \in \mathbb{N}$, there is a lattice $\Theta$ such that $\Gamma \supset \Theta \supset \Lambda, p(\Theta)=p(\Gamma)$ and $\xi_{\Theta}=\xi$.

Proof. In view of Proposition 1.3, we may assume without loss of generality that $\Gamma=\left\{c\left(j_{3} / k\right) b\left(j_{2}\right) a\left(j_{1}\right) \mid j_{1}, j_{2}, j_{3} \in \mathbb{Z}\right\}$ for some $k \in \mathbb{N}$. It follows from the condition of the lemma that there are positive integers $k_{1}, k_{2}, k_{3}$ such that $k=k_{1} k_{2}, \xi=$ $1 / k_{2}$ and $\xi_{\Lambda}=k_{3} / k_{2}$. Let $\Theta$ denote the group generated by $\Lambda$ and the lattice $\left\{c\left(j_{3} / k_{2}\right) b\left(j_{2}\right) a\left(j_{1}\right) \mid j_{1}, j_{2}, j_{3} \in \mathbb{Z}\right\}$. Then $\Theta$ is a lattice in $H_{3}(\mathbb{R})$. Of course, $\Gamma \supset \Theta \supset \Lambda$ and $p(\Theta)=p(\Gamma)$. It is easy to see that

$$
\xi_{\Theta} \mathbb{Z}=\xi_{\Lambda} \mathbb{Z}+\frac{1}{k_{2}} \mathbb{Z}=\frac{1}{k_{2}} \mathbb{Z}
$$

i.e. $\xi_{\Theta}=\xi$.

\footnotetext{
${ }^{3}$ Indeed, the flow $F: \mathbb{R} \times H_{3}(\mathbb{R}) / \Gamma \ni(t, g \Gamma) \mapsto c(t) g \Gamma \in H_{3}(\mathbb{R}) / \Gamma$ is periodic with period $\xi_{\Gamma}$. Hence the Borel space $O$ of $F$-orbits is standard. On the other hand, $O$ is isomorphic as a Borel space to $H_{3}(\mathbb{R}) / p^{-1}(p(\Gamma))$. The later space is, in turn, isomorphic to the Borel space $\mathbb{R}^{2} / p(\Gamma)$. Hence $\mathbb{R}^{2} / p(\Gamma)$ endowed with the natural quotient Borel structure is standard. Hence $p(\Gamma)$ is closed in $\mathbb{R}^{2}$ by $[\mathrm{Ma} 2]$.
} 
Remark 1.6. It is straightforward to verify that for each lattice $\Gamma$ in $H_{3}(\mathbb{R})$, we have $k_{\Gamma}=\frac{|\operatorname{det} A|}{\xi_{\Gamma}}$, where the matrix $A$ is defined by $p(\Gamma)=A\left(\mathbb{Z}^{2}\right)$. Indeed, since $[\Gamma, \Gamma]=\left\{c\left(k_{\Gamma} \xi_{\Gamma} m\right) \mid m \in \mathbb{Z}\right\}$, it follows that $\left[\gamma_{1}, \gamma_{2}\right]=c\left( \pm k_{\Gamma} \xi_{\Gamma}\right)$ for each pair of elements $\gamma_{1}, \gamma_{2} \in \Gamma$ whose images $p\left(\gamma_{1}\right)$ and $p\left(\gamma_{2}\right)$ in $\mathbb{R}^{2}$ generate the entire lattice $p(\Gamma)$.

Remark 1.7. There is a natural action of $\operatorname{Aut}\left(H_{3}(\mathbb{R})\right)$ on $\widehat{H_{3}(\mathbb{R})}$. It is not difficult to compute that $\pi_{\alpha, \beta} \circ \theta_{A, \xi, \eta}=\pi_{\xi_{1} \alpha+\xi_{2} \beta, \eta_{1} \alpha+\eta_{2} \beta}$ and $\pi_{\gamma} \circ \theta_{A, \xi, \eta} \sim \pi_{\gamma \cdot \operatorname{det} A}$.

\section{Odometer ACTIONS OF LOCALLY COMPACT GROUPS}

In this section we introduce the concept of odometer action for locally compact second countable groups. We study some general structural properties of these actions and discuss when they are free.

Let $G$ and $K$ be locally compact second countable groups. Let $T=\left(T_{g}\right)_{g \in G}$ be an action of $G$ on a standard Borel space $Z$. A Borel map $c: G \times Z \rightarrow K$ is called a cocycle of $T$ if

$$
c\left(g_{1} g_{2}, z\right)=c\left(g_{1}, T_{g_{2}} z\right) c\left(g_{2}, z\right)
$$

for all $g_{1}, g_{2} \in G$ and $z \in Z$. Two cocycles $c, c^{\prime}: G \times Z \rightarrow K$ are cohomologous if there is a Borel map $d: Z \rightarrow K$ such that $d(g z) c(g, z) d(z)^{-1}=c^{\prime}(g, z)$ for all $g \in G$ and $z \in Z$. Given a cocycle $c$, we can define an action $T^{c}=\left(T_{g}^{c}\right)_{g \in G}$ of $G$ on $Z \times K$ by setting

$$
T_{g}^{c}(z, k)=\left(T_{g} z, c(g, z) k\right) .
$$

It is called the $c$-skew product extension of $T$. If $c$ is cohomologous to another cocycle $c^{\prime}$ of $T$ then the actions $T^{c}$ and $T^{c^{\prime}}$ of $G$ are isomorphic. If $T$ preserves a $\sigma$-finite measure $\mu$ then $T^{c}$ preserves the $\sigma$-finite measure $\mu \times \lambda_{K}$, where $\lambda_{K}$ denotes a left Haar measure on $K$. If $T^{c}$ is ergodic then $c$ is called ergodic.

Let $N$ be a closed subgroup of $G$. Let the two groups be unimodular. Fix a Borel cross-section $s: G / N \rightarrow G$ of the natural projection $G \rightarrow G / N$ such that $s(N)=1$. Then a cocycle $h_{s}: G \times G / N \rightarrow N$ of the natural $\sigma$-finite (Haar) measure preserving $G$-action on $G / N$ by rotations is well defined by the formula $h_{s}(g, x):=s(g x)^{-1} g s(x)$. The cohomology class of $h_{s}$ does not depend on the choice of $s$. Below we will call $h_{s}$ a choice-cocycle. It is easy to see that the $h_{s}$-skew product extension is isomorphic to the action of $G$ on itself by left translations.

Remark 2.0. Given a cocycle $c: G \times G / N \rightarrow K$, a continuous group homomorphism $\phi: N \rightarrow K$ is well defined by $\phi(n):=c(n, N)$. Consider a mapping $d: G / N \ni x \mapsto$ $d(x):=c(s(x), N) \in K$. Then we have

$$
\begin{aligned}
c\left(g_{1}, g_{2} N\right) & =c\left(g_{1} g_{2}, N\right) c\left(g_{2}, N\right)^{-1} \\
& =c\left(s\left(g_{1} g_{2} N\right) h_{s}\left(g_{1} g_{2}, N\right), N\right) c\left(s\left(g_{2} N\right) h_{s}\left(g_{2}, N\right), N\right)^{-1} \\
& =d\left(g_{1} g_{2} N\right) \phi\left(h_{s}\left(g_{1} g_{2}, N\right) h_{s}\left(g_{2}, N\right)^{-1}\right) d\left(g_{2} N\right)^{-1} \\
& =d\left(g_{1} g_{2} N\right) \phi\left(h_{s}\left(g_{1}, g_{2} N\right)\right) d\left(g_{2} N\right)^{-1} .
\end{aligned}
$$

In other words, $c$ is cohomologous to the cocycle $\phi \circ h_{s}$. Thus, the cohomology class of $c$ is determined completely by $\phi$ (or, more precisely, by the conjugacy class of $\phi$ ). 
Remark 2.1. If $K$ is Abelian then the essential range ${ }^{4} E(c)$ of $c$ is the closure of the subgroup $\phi(N)$ in $K$. Indeed, this follows from the fact that $h_{s}$ is ergodic (in fact, the corresponding $h_{s}$-skew product is transitive).

Let $\Gamma_{1} \supset \Gamma_{2} \supset \cdots$ be a nested sequence of lattices in $G$. Then we consider a projective sequence of homogeneous $G$-spaces

$$
G / \Gamma_{1} \leftarrow G / \Gamma_{2} \leftarrow \cdots
$$

All arrows are $G$-equivariant and onto. Denote by $X$ the projective limit of this sequence. Then $X$ is a locally compact second countable $G$-space. Indeed, $G / \Gamma_{1}$ is locally compact and every arrow in (2-1) is a finite-to-one mapping. ${ }^{5}$ The $G$-action is minimal and uniquely ergodic. The only invariant probability measure $\mu$ on $X$ is the projective limit of the probability Haar measures on $G / \Gamma_{n}$.

Definition 2.2. We call the dynamical system $(X, \mu, G)$ a $G$-odometer ${ }^{6}$. If $\Gamma_{n}$ is normal in $\Gamma_{1}$ for each $n$ then we call the corresponding $G$-odometer normal.

We note that $X$ is compact if and only if each $\Gamma_{n}$ is uniform, i.e. co-compact in $G$.

To show a 'product structure' on $X$ we denote by $s_{j}: \Gamma_{j-1} / \Gamma_{j} \rightarrow \Gamma_{j-1}$ a Borel cross-section of the natural projection $\Gamma_{j-1} \rightarrow Y_{j}:=\Gamma_{j-1} / \Gamma_{j}$ such that $s_{j}\left(\Gamma_{j}\right)=1$. For consistency of the notation we set $\Gamma_{0}=G$. Let $h_{j}: \Gamma_{j-1} \times$ $Y_{j} \ni(g, y) \mapsto h_{j}(g, y) \in \Gamma_{j}$ stand for the corresponding choice-cocycle. Then $g s_{j}(y)=s_{j}(g y) h_{j}(g, y)$ for all $g \in \Gamma_{j-1}$ and $y \in Y_{j}$. Denote by $\psi_{j}$ a mapping

$$
G / \Gamma_{1} \times \Gamma_{1} / \Gamma_{2} \times \cdots \times \Gamma_{j-1} / \Gamma_{j} \ni\left(y_{1}, \ldots, y_{j}\right) \mapsto s_{1}\left(y_{1}\right) s_{2}\left(y_{2}\right) \cdots s_{j}\left(y_{j}\right) \Gamma_{j} \in G / \Gamma_{j}
$$

for $j>1$ and $\psi_{1}:=\mathrm{id}$. It is easy to see that each $\psi_{j}$ is a Borel isomorphism. We obtain the following infinite commutative diagram

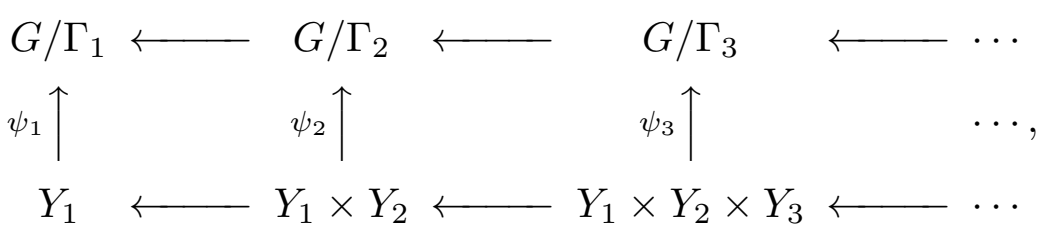

where the horizontal arrows in the lower lines are natural projections. Therefore the sequence of mappings $\left(\psi_{j}^{-1}\right)_{j=1}^{\infty}$ generates a Borel isomorphism $\psi$ of $X:=$ proj $\lim _{j} G / \Gamma_{j}$ onto $Y:=Y_{1} \times Y_{2} \times \cdots$. Since $X$ is a $G$-space, $Y$ is also a $G$-space. We thus obtain the following proposition.

Proposition 2.3. The G-space $X$ is Borel isomorphic to the infinite Cartesian product

$$
Y=G / \Gamma_{1} \times \Gamma_{1} / \Gamma_{2} \times \Gamma_{2} / \Gamma_{3} \times \cdots
$$

in such a way that

$$
g \cdot\left(y_{1}, y_{2}, y_{3}, \ldots\right)=\left(g y_{1}, h_{1}\left(g, y_{1}\right) y_{2}, h_{2}\left(h_{1}\left(g, y_{1}\right), y_{2}\right) y_{3}, \ldots\right)
$$

\footnotetext{
${ }^{4}$ For the definition of the essential range of a cocycle we refer to [Sc].

${ }^{5}$ We note that $\Gamma_{n}$ is of finite index in $\Gamma_{1}$.

${ }^{6}$ Thus the $G$-odometers are ergodic. Cf. [CoPe], where the case of discrete and finitely generated $G$ was under consideration.
} 
for all $\left(y_{1}, y_{2} \ldots\right) \in Y$ and $g \in G$.

We recall that given a lattice $\Gamma$ in $G$ and a measure preserving action $V=\left(V_{\gamma}\right)_{\gamma \in \Gamma}$ of $\Gamma$ on a standard probability space $(Z, \kappa)$, there is a natural induced measure preserving $G$-action [Ma1] on the product space $(G / \Gamma \times Z, \lambda \times \kappa)$ :

$$
g(x, z):=\left(g x, V_{h_{s}(g, x)} z\right),
$$

where $\lambda$ is the probability Haar measure on $G / \Gamma$ and $h_{s}: G \times G / \Gamma \rightarrow \Gamma$ is the 1-cocycle corresponding to a cross-section $s: G / \Gamma \rightarrow G$. We denote this action by $\operatorname{Ind}_{\Gamma}^{G}(V)$. The isomorphism class of $\operatorname{Ind}_{\Gamma}^{G}(V)$ does not depend on a particular choice of $s$.

Let $K$ be a compact metric group and let $\alpha: \Gamma \times Z \rightarrow K$ be a cocycle of $V$. Then we can define an induced cocycle $\widetilde{\alpha}: G \times(G / \Gamma \times Z) \rightarrow K$ of $\operatorname{Ind}_{\Gamma}^{G}(V)$ by setting

$$
\widetilde{\alpha}(g,(x, z)):=\alpha\left(h_{s}(g, x), z\right) .
$$

The next lemma is about basic properties of induced cocycles. They follow easily from the definitions and we state this lemma without proof.

\section{Lemma 2.4.}

(i) $\operatorname{Ind}_{\Gamma}^{G}\left(V^{\alpha}\right)=\left(\operatorname{Ind}_{\Gamma}^{G}(V)\right)^{\widetilde{\alpha}}$. In particular, $\widetilde{\alpha}$ is ergodic if and only if $\alpha$ is ergodic.

(ii) Two cocycles $\alpha, \beta$ of $V$ are cohomologous if and only if the induced cocycles $\widetilde{\alpha}$ and $\widetilde{\beta}$ of $\operatorname{Ind}_{\Gamma}^{G}(V)$ are cohomologous.

(iii) For each cocycle $\delta$ of $\operatorname{Ind}_{\Gamma}^{G}(V)$, there exists a cocycle $\alpha$ of $V$ such that $\delta$ is cohomologous to $\widetilde{\alpha}$.

(iv) Let $\mathfrak{F}$ be a factor of $V$ and let $\widetilde{\mathfrak{F}}:=\operatorname{Ind}_{\Gamma}^{G}(\mathfrak{F})$ denote the corresponding factor of $\operatorname{Ind}_{\Gamma}^{G}(V)$. Then $\widetilde{\alpha}$ is $\widetilde{\mathfrak{F}}$-measurable if and only if $\alpha$ is $\mathfrak{F}$-measurable.

The next statement follows easily from Proposition 2.3.

Corollary 2.5. Let $T$ be a G-odometer associated with a sequence $\Gamma_{1} \supset \Gamma_{2} \supset \ldots$ of lattices in $G$. Let $V$ denote the $\Gamma_{1}$-odometer associated with the sequence $\Gamma_{2} \supset$ $\Gamma_{3} \supset \cdots$ of lattices in $\Gamma_{1}$. Then $\operatorname{Ind}_{\Gamma_{1}}^{G}(V)=T$.

We now find some conditions under which a $G$-odometer is free. Let $X$ denote the space of an odometer $T$. Every point $x \in X$ can be written as an infinite sequence $x=\left(g_{1} \Gamma_{1}, g_{2} \Gamma_{2}, \ldots\right)$ for some $g_{1} \in G, g_{2} \in g_{1} \Gamma_{1}, g_{3} \in g_{2} \Gamma_{2}, \ldots$ Denote by $S_{x}$ the stability group of $T$ at $x$.

Proposition 2.6. Let $x=\left(g \Gamma_{1}, g \gamma_{1} \Gamma_{2}, g \gamma_{1} \gamma_{2} \Gamma_{3}, \ldots\right) \in X$ for some $g \in H_{3}(\mathbb{R})$ and $\gamma_{n} \in \Gamma_{n}, n \in \mathbb{N}$. Then $S_{x}=g\left(\bigcap_{n=1}^{\infty} \gamma_{1} \cdots \gamma_{n-1} \Gamma_{n} \gamma_{n-1}^{-1} \cdots \gamma_{1}^{-1}\right) g^{-1}$. Therefore $T$ is free if and only if

$$
\bigcap_{n=1}^{\infty} \gamma_{1} \cdots \gamma_{n-1} \Gamma_{n} \gamma_{n-1}^{-1} \cdots \gamma_{1}^{-1}=\{1\}
$$

for a.e. $x$, i.e. for a.e. sequence $\left(\gamma_{1} \Gamma_{2}, \gamma_{2} \Gamma_{3}, \ldots\right) \in \prod_{n \in \mathbb{N}} \Gamma_{n} / \Gamma_{n+1}$, where the infinite product space is endowed with the infinite product measure obtained from equi-distributions on the factors. Hence $T$ is free if and only if the $\Gamma_{1}$-odometer 
associated with the sequence $\Gamma_{2} \supset \Gamma_{3} \supset \cdots$ is free. The restriction of $T$ to the center of $G$ is free if and only if the intersection of the center with the group $\bigcap_{n=1}^{\infty} \Gamma_{n}$ is trivial.

Proof. Since $T_{h} x=\left(h g \Gamma_{1}, h g \gamma_{1} \Gamma_{2}, h g \gamma_{1} \gamma_{2} \Gamma_{3}, \ldots\right)$, it follows that $T_{h} x=x$ if and only if $h \in \bigcap_{n=1}^{\infty} g \gamma_{1} \cdots \gamma_{n-1} \Gamma_{n} \gamma_{n-1}^{-1} \cdots \gamma_{1}^{-1} g^{-1}$.

Corollary 2.7. Let $(X, \mu, T)$ be the odometer associated with a sequence $\Gamma_{1} \supset$ $\Gamma_{2} \supset \cdots$ of lattices in $G$.

(i) If $\Gamma_{n}$ is normal in $\Gamma_{1}$ for each $n$, then $T$ is free if and only if $\bigcap_{n=1}^{\infty} \Gamma_{n}=\{1\}$.

(ii) Let $\widetilde{\Gamma}_{n}:=\bigcap_{\gamma \in \Gamma_{1}} \gamma \Gamma_{n} \gamma^{-1}$. Then $\widetilde{\Gamma}_{n}$ is a subgroup of finite index in $\Gamma_{n}$. It is normal in $\Gamma_{1}$. Moreover, $\Gamma_{1} \supset \widetilde{\Gamma}_{2} \supset \widetilde{\Gamma}_{3} \supset \cdots$. Denote by $(\widetilde{X}, \widetilde{\mu}, \widetilde{T})$ the corresponding normal $G$-odometer. We call it the normal cover of $(X, \mu, T)$. The sequence of natural $G$-equivariant maps $G / \widetilde{\Gamma}_{n} \rightarrow G / \Gamma_{n}$ generates a factor map $(\widetilde{X}, \widetilde{\mu}, \widetilde{T}) \rightarrow(X, \mu, T)$. Thus every odometer is a factor of a normal odometer. Moreover, if $\bigcap_{n=1}^{\infty} \Gamma_{n}=\{1\}$ then $\bigcap_{n=1}^{\infty} \widetilde{\Gamma}_{n} \subset \bigcap_{n=1}^{\infty} \Gamma_{n}$ and the corresponding normal cover is free ${ }^{7}$. Furthermore, $\bigcap_{n=1}^{\infty} \widetilde{\Gamma}_{n} \cap\{c(t) \mid$ $t \in \mathbb{R}\}=\bigcap_{n=1}^{\infty} \Gamma_{n} \cap\{c(t) \mid t \in \mathbb{R}\}$.

Remark 2.8. If there is a F $\varnothing$ lner sequence $\left(F_{n}\right)_{n=1}^{\infty}$ in $G$ such that $G=\bigsqcup_{\gamma \in \Gamma_{n}} F_{n-1} \gamma$ and $F_{n}=\bigsqcup_{\gamma \in C_{n}} F_{n-1} \gamma$ for some finite subset $C_{n} \subset \Gamma_{n}$ and each $n>0$ then the corresponding $G$-odometer is the $(C, F)$-action associated with $\left(F_{n-1}, C_{n}\right)_{n=1}^{\infty}$ (see [Da3]). Every $(C, F)$-action is free. We note that $\Gamma_{n}=C_{n} \Gamma_{n+1}$ and $c \Gamma_{n+1} \cap$ $c^{\prime} \Gamma_{n+1}=\emptyset$ whenever $c \neq c^{\prime} \in C_{n}$. Hence $\# C_{n}$ is the index of $\Gamma_{n+1}$ in $\Gamma_{n}$.

\section{FreEnESS FOR HeISENBERG ODOMETERS}

In this section we consider in detail the problem of when $H_{3}(\mathbb{R})$-odometers are free. Some criteria of the freeness are found. A couple of counterexamples are also given here.

Theorem 3.1. Let $T$ be the $H_{3}(\mathbb{R})$-odometer associated with a sequence $\Gamma_{1} \supset \Gamma_{2} \supset$ .. of lattices in $H_{3}(\mathbb{R})$. Then $T$ is free if and only if $\{c(t) \mid t \in \mathbb{R}\} \cap \bigcap_{n=1}^{\infty} \Gamma_{n}=\{1\}$.

Proof. Let $(X, \mathfrak{B}, \mu)$ denote the (standard probability) space of $T$. Suppose that $\{c(t) \mid t \in \mathbb{R}\} \cap \bigcap_{n=1}^{\infty} \Gamma_{n}=\{1\}$. It follows from Proposition 2.6 that the restriction of $T$ to the center of $H_{3}(\mathbb{R})$ is free. Let $\mathfrak{S}$ stand for the space of closed subgroups in $H_{3}(\mathbb{R})$. It is a standard Borel space when endowed with the Fell topology [Fe]. Denote by $S_{x}$ the stability group of $T$ at a point $x \in X$. If follows from Proposition 2.6 that $S_{x}$ is countable. The map $X \ni x \mapsto S_{x} \in \mathfrak{S}$ is Borel [AGH]. It is easy to see that

$$
S_{T_{g} x}=g S_{x} g^{-1} \quad \text { for all } \quad g \in H_{3}(\mathbb{R}) \quad \text { and } x \in X .
$$

We let $\widetilde{S}_{x}:=S_{x} \cap\{c(t) \mid t \in \mathbb{R}\}$. It follows from (3-1) that the Borel map $X \ni x \mapsto \widetilde{S}_{x} \in \mathfrak{S}$ is invariant under $T$ and hence constant almost everywhere. Since the restriction of $T$ to the center of $H_{3}(\mathbb{R})$ is free, we obtain that $\widetilde{S}_{x}$ is trivial

\footnotetext{
${ }^{7}$ See also $[\mathrm{CoPe}]$ for a analogous claim for a subclass of discrete $G$.
} 
for almost all $x$. According to [GoSi] and $[\mathrm{CRa}]^{8}$, there is $S \in \mathfrak{S}$ and a Borel map $\phi: X \rightarrow G$ such that

$$
\phi(x) S_{x} \phi(x)^{-1}=S \quad \text { for almost all } x .
$$

Since $S$ is countable and closed and $S \cap\{c(t) \mid t \in \mathbb{R}\}=\{1\}$, it follows that either $S$ is trivial or there are a countable subgroup $D$ in $\mathbb{R}$, a homomorphism $\varphi: D \rightarrow \mathbb{R}$ and three reals $\theta_{1}, \theta_{2}, \theta_{3}$ such that $\theta_{1}^{2}+\theta_{2}^{2} \neq 0$ and $^{9}$

$$
S=\left\{c\left(\varphi(d) \theta_{3}\right) b\left(d \theta_{2}\right) a\left(d \theta_{1}\right) \mid d \in D\right\} .
$$

Suppose that $S$ is non-trivial. We note that (3-2) defines $\phi$ up to multiplying with an element of the normalizer $N(S)$ of $S$ in $H_{3}(\mathbb{R})$. We recall that $N(S):=\{g \in$ $\left.H_{3}(\mathbb{R}) \mid g S g^{-1}=S\right\}$. It is straightforward to verify that

$$
N(S)=\left\{c\left(t_{1} \theta_{3}\right) b\left(t_{2} \theta_{2}\right) a\left(t_{2} \theta_{1}\right) \mid t_{1}, t_{2} \in \mathbb{R}\right\} .
$$

Indeed, an element $c\left(r_{3}\right) b\left(r_{2}\right) a\left(r_{1}\right)$ of $H_{3}(\mathbb{R})$ belongs to $N(S)$ if and only if the commutator $\left[c\left(r_{3}\right) b\left(r_{2}\right) a\left(r_{1}\right), c\left(\varphi(d) \theta_{3}\right) b\left(d \theta_{2}\right) a\left(d \theta_{1}\right)\right]$ belongs to $S$ for all $d \in D$. Thus $c\left(r_{1} d \theta_{2}-r_{2} d \theta_{1}\right) \in S$. This is possible only if $r_{1} \theta_{2}-r_{2} \theta_{1}=0$, i.e. the vector $\left(r_{1}, r_{2}\right) \in \mathbb{R}^{2}$ belongs to the line generated by $\left(\theta_{1}, \theta_{2}\right)$, as desired. We note that $N(S)$ is a closed normal subgroup of $H_{3}(\mathbb{R})$ and $H_{3}(\mathbb{R}) / N(S)=\mathbb{R}$. Moreover, the natural projection can be written in the following way

$$
H_{3}(\mathbb{R}) / N(S) \ni g N(S)=b\left(-t \theta_{1}\right) a\left(t \theta_{2}\right) N(S) \mapsto t \in \mathbb{R},
$$

where the real $t$ is uniquely determined by the $\operatorname{coset} g N(S)$. We now let $\widetilde{\phi}(x):=$ $\phi(x) N(S)$. Then $\widetilde{\phi}$ is a well defined $(\bmod 0)$ Borel map from $X$ to $H_{3}(\mathbb{R}) / N(S)=\mathbb{R}$. Substituting (3-2) into (3-1) we deduce that

$$
\phi\left(T_{g} x\right)^{-1} S \phi\left(T_{g} x\right)=g^{-1} \phi(x)^{-1} S \phi(x) g
$$

and hence

$$
\phi(x) g \phi\left(T_{g} x\right)^{-1} \in N(S)
$$

for a.a. $x$. Therefore $-\widetilde{\phi}(x)+\widetilde{\phi}\left(T_{g} x\right) \in g N(S)$ for a.a. $x$ for each $g \in H_{3}(\mathbb{R})$. It follows that $\widetilde{\phi}$ is invariant under $\left(T_{g}\right)_{g \in N(S)}$. Denote by $q:(X, \mu) \rightarrow(Y, \nu)$ the $\left(T_{g}\right)_{g \in N(S)}$-ergodic decomposition. Then there are an ergodic flow $V=\left(V_{t}\right)_{t \in \mathbb{R}}$ on

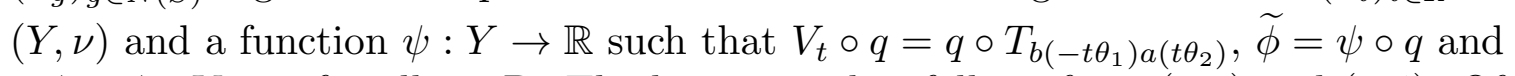
$-\psi+\psi \circ V_{t}=t$ for all $t \in \mathbb{R}$. The latter equality follows from (3-3) and (3-4). Of course, such a function $\psi$ does not exist. Thus we obtain a contradiction. Hence $S$ is trivial, i.e. $T$ is free.

The converse follows from Proposition 2.6 and from the obvious remark that if $T$ is free then the restriction of $T$ to the center of $H_{3}(\mathbb{R})$ is also free.

The next claim follows from Proposition 2.6, Theorem 3.1 and Corollary 2.7(ii).

\footnotetext{
${ }^{8}$ The stability groups of an ergodic probability preserving action of a simple connected nilpotent Lie group are conjugate (in a Borel way) over a conull subset.

${ }^{9}$ We note that $D$ is not necessarily closed in $\mathbb{R}$. Consider, for instance, the closed subgroup $S=\{c(n) a(m \tau+n) \mid m, n \in \mathbb{Z}\}$ in $H_{3}(\mathbb{R})$, where $\tau$ is an irrational number.
} 


\section{Corollary 3.2.}

(i) A Heisenberg odometer is free if and only if its restriction to the center is free.

(ii) A Heisenberg odometer is free if and only if its normal cover is free.

It follows from the next example that the condition $\{c(t) \mid t \in \mathbb{R}\} \cap \bigcap_{n=1}^{\infty} \Gamma_{n}=$ $\{1\}$ does not imply that $\bigcap_{n=1}^{\infty} \Gamma_{n}=\{1\}$.

Example 3.3. Let $\Gamma_{n}:=\left\{c\left(n ! i_{3}\right) b\left(n ! i_{2}\right) a\left(i_{1}\right) \mid i_{1}, i_{2}, i_{3} \in \mathbb{Z}\right\}$. Then $\Gamma_{n}$ is a lattice in $H_{3}(\mathbb{R}), \Gamma_{1} \supset \Gamma_{2} \supset \cdots$ and $\{c(t) \mid t \in \mathbb{R}\} \cap \bigcap_{n=1}^{\infty} \Gamma_{n}=\{1\}$. On the other hand, $\bigcap_{n=1}^{\infty} \Gamma_{n}=\left\{a\left(i_{1}\right) \mid i_{1} \in \mathbb{Z}\right\}$.

Consider now the case of normal Heisenberg odometers.

Proposition 3.4. If $T$ is a normal free Heisenberg odometer then $\bigcap_{n=1}^{\infty} p\left(\Gamma_{n}\right)=$ $\{0\}$.

Proof. Suppose that $\bigcap_{n=1}^{\infty} p\left(\Gamma_{n}\right) \neq\{0\}$. Then there is $g \in H_{3}(\mathbb{R}) \backslash\{c(t) \mid t \in \mathbb{R}\}$ and a sequence $\left(r_{n}\right)_{n=1}^{\infty}, r_{n} \in \mathbb{R}$, such that $g c\left(r_{n}\right) \in \Gamma_{n}$ for all $n \in \mathbb{N}$. Since $g$ does not belong to the center of $H_{3}(\mathbb{R})$, there is $\gamma$ in $\Gamma_{1}$ such that $g$ and $\gamma$ do not commute (see Corollary 1.4). Therefore $\gamma g \gamma^{-1}=g c(t)$ for some $t \neq 0$. This yields $g c\left(r_{n}\right) c(t)=\gamma g c\left(r_{n}\right) \gamma^{-1} \in \gamma \Gamma_{n} \gamma^{-1}=\Gamma_{n}$ and hence $c(t) \in \Gamma_{n}$ for all $n$. This contradicts to the fact that $\bigcap_{n=1}^{\infty} \Gamma_{n}=\{1\}$.

It may seem that the condition $\bigcap_{n=1}^{\infty} p\left(\Gamma_{n}\right)=\{0\}$ is satisfied for every odometer with $\bigcap_{n=1}^{\infty} \Gamma_{n}=\{1\}$. In the next example we show that this is not true. Hence the corresponding odometer is not normal.

Example 3.5. Let $k_{1}:=1$ and $k_{n}:=k_{n-1}\left(k_{n-1}+1\right)$. Let

$$
\left.\Gamma_{n}:=\left\{c\left(k_{n}^{2} i_{3}+k_{n} i_{1}\right) b\left(k_{n}^{2} i_{2}\right) a\left(i_{1}\right)\right) \mid i_{1}, i_{2}, i_{3} \in \mathbb{Z}\right\} .
$$

Then $\Gamma_{n}$ is a lattice in $H_{3}(\mathbb{R}), \Gamma_{1} \supset \Gamma_{2} \supset \cdots$ and $\bigcap_{n=1}^{\infty} \Gamma_{n}=\{1\}$. On the other hand, $p\left(\Gamma_{n}\right)=\left(\begin{array}{cc}1 & 0 \\ 0 & k_{n}^{2}\end{array}\right) \mathbb{Z}^{2}$ and hence $\bigcap_{n=1}^{\infty} p\left(\Gamma_{n}\right)=\left(\begin{array}{ll}1 & 0 \\ 0 & 0\end{array}\right) \mathbb{Z}^{2} \neq\{0\}$.

\section{Spectral analysis for homogeneous actions of $H_{3}(\mathbb{R})$ ON NIL-MANIFOLDS}

In this section we review

(a) Kirillov's orbit method to get a description of the unitary dual for the Heisenberg group $[\mathrm{Ki}]$ and

(b) the Howe-Richardson formula for the multiplicities of the irreducible components in spectral decomposition of homogeneous $H_{3}(\mathbb{R})$-spaces [Ho], [Ri].

Given a lattice $\Gamma$ in $H_{3}(\mathbb{R})$, the quotient space $H_{3}(\mathbb{R}) / \Gamma$ is called a nil-manifold. Consider the quasi-regular representation $U$ of $H_{3}(\mathbb{R})$ in $L^{2}\left(H_{3}(\mathbb{R}) / \Gamma\right)$, i.e. the Koopman representation of $H_{3}(\mathbb{R})$ corresponding to the natural action of this group by rotations on $H_{3}(\mathbb{R}) / \Gamma$. Since $\Gamma$ is uniform, it follows that there is a countable family $\Theta$ of irreducible unitary representations of $H_{3}(\mathbb{R})$ and positive integers $m_{W}$, $W \in \Theta$, such that

$$
U=\bigoplus_{W \in \Theta} \bigoplus_{j=1}^{m_{W}} W
$$


The family $\Theta$ (or, rather their unitary equivalence classes) and the map $\Theta \ni W \mapsto$ $m_{W}$ are defined uniquely by $U$. Our purpose is to describe them explicitly. Partition $\Theta$ into two subsets $\Theta_{1}$ and $\Theta_{\infty}$ consisting of the 1-dimensional representations and the $\infty$-dimensional representations of $H_{3}(\mathbb{R})$ respectively.

If $\pi_{\alpha, \beta} \in \Theta_{1}$ for some $(\alpha, \beta) \in \mathbb{R}^{2}$, then there is $f \in L^{2}\left(H_{3}(\mathbb{R}) / \Gamma\right)$ such that $f \circ T_{g}^{-1}=e^{2 \pi i\left(\alpha t_{1}+\beta t_{2}\right)} f$ for all $g \in \mathbb{H}_{3}(\mathbb{R})$, where $g=c\left(t_{3}\right) b\left(t_{2}\right) a\left(t_{1}\right)$. In particular, $f$ is invariant under the action of the center. We recall (see the footnote No 3 ) that the natural quotient map

$$
H_{3}(\mathbb{R}) / \Gamma \rightarrow H_{3}(\mathbb{R}) / p^{-1}(p(\Gamma))=\mathbb{R}^{2} / p(\Gamma)
$$

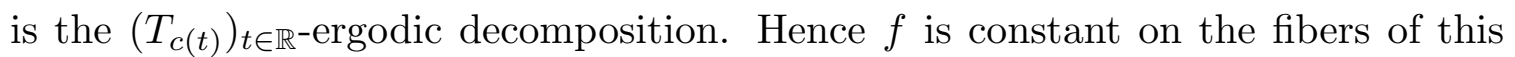
quotient map. Moreover, $f$ is an eigenfunction for the $\mathbb{R}^{2}$-action by rotations on $\mathbb{R}^{2} / p(\Gamma)$. The converse is also true. Indeed, if $A \in \mathrm{GL}_{2}(\mathbb{R})$ then $\left(A^{*}\right)^{-1} \mathbb{Z}^{2}$ are the eigenvalues of the $\mathbb{R}^{2}$-actions by rotations on $\mathbb{R}^{2} / A\left(\mathbb{Z}^{2}\right)$. If $p(\Gamma)=A\left(\mathbb{Z}^{2}\right)$ for some matrix $A \in \mathrm{GL}_{2}(\mathbb{R})$ then we denote by $p(\Gamma)^{*}$ the dual lattice $\left(A^{*}\right)^{-1} \mathbb{Z}^{2}$ in $\mathbb{R}^{2}$. It is easy to see that the dual lattice is well defined, i.e. does not depend on the choice of $A$. We now obtain that

$$
\Theta_{1}=\left\{\pi_{\alpha, \beta} \mid(\alpha, \beta) \in p(\Gamma)^{*}\right\}
$$

Since $T$ is ergodic (it is even transitive), it follows that the corresponding multiplicities are all 1, i.e. $m_{W}=1$ whenever $W \in \Theta_{1}$.

Now let us calculate $\Theta_{\infty}$. Fix a $p(\Gamma)$-fundamental parallelepiped $\Delta$ in $\mathbb{R}^{2}$. Consider the map

$$
\Psi: H_{3}(\mathbb{R}) / \Gamma \ni g \Gamma \mapsto\left(\left(t_{1}, t_{2}\right)+p(\Gamma), t_{3}+\xi_{\Gamma} \mathbb{Z}\right) \in H_{3}(\mathbb{R}) / p^{-1}(p(\Gamma)) \times \mathbb{R} / \xi_{\Gamma} \mathbb{Z},
$$

where the reals $t_{1}, t_{2}, t_{3}$ are determined by the formula $g \in c\left(t_{3}\right) b\left(t_{2}\right) a\left(t_{1}\right) \Gamma$ with $t_{1}, t_{2} \in \Delta$. This map conjugates the $H_{3}(\mathbb{R})$-action on the nilmanifold $X:=H_{3}(\mathbb{R}) / \Gamma$ with the $\mathbb{R} / \xi_{\Gamma} \mathbb{Z}$-group extension of the $H_{3}(\mathbb{R})$-action on the quotient space $Y:=$ $H_{3}(\mathbb{R}) / p^{-1}(p(\Gamma))$. We let $\eta_{n}(w)=e^{2 \pi i n w / \xi_{\Gamma}}, n \in \mathbb{R}, w \in\left[0, \xi_{\Gamma}\right)=\mathbb{R} / \xi_{\Gamma} \mathbb{Z}$. Since the group characters $\left\{\eta_{n} \mid n \in \mathbb{Z}\right\}$ form an orthonormal basis in $L^{2}\left(\mathbb{R} / \xi_{\Gamma} \mathbb{Z}\right)$, we obtain a decomposition of $L^{2}(X)$ into the following orthogonal sum

$$
L^{2}(X)=\bigoplus_{n \in \mathbb{Z}} L^{2}(Y) \otimes \eta_{n}
$$

It is easy to see that every subspace $L^{2}(Y) \otimes \eta_{n}$ is invariant under $U$. We note that if $\Psi(g \Gamma)=\left(y, t_{3}+\xi_{\Gamma} \mathbb{Z}\right)$ for some $g \in H_{3}(\mathbb{R}), y \in Y$ and $t_{3} \in \mathbb{R}$ then $\Psi(c(t) g \Gamma)=$ $\left(y, t+t_{3}+\xi_{\Gamma} \mathbb{Z}\right)$ for all $t \in \mathbb{R}$. This implies that

$$
U(c(t))\left(f \otimes \eta_{n}\right)=e^{2 \pi i n t / \xi_{\Gamma}} f \otimes \eta_{n}
$$

for all $f \in L^{2}(Y), n \in \mathbb{Z}$. Hence $n / \xi_{\Gamma} \in \Theta_{\infty}$ and

$$
U \uparrow\left(L^{2}(Y) \otimes \eta_{n}\right) \subset \bigoplus_{1}^{m_{\pi_{n} / \xi_{\Gamma}}} \pi_{n / \xi_{\Gamma}}
$$


if $n \neq 0$. Of course, $U \uparrow\left(L^{2}(Y) \otimes 1\right)=\bigoplus_{W \in \Theta_{1}} W$. Therefore we deduce from (4-1), (4-2) and (4-3) that

$$
\Theta_{\infty}=\left\{\pi_{\gamma} \mid \gamma \in \xi_{\Gamma}^{-1} \mathbb{Z}, \gamma \neq 0\right\}
$$

and

$$
U \uparrow\left(L^{2}(Y) \otimes \eta_{n}\right)=\bigoplus_{1}^{m_{\pi_{n / \xi_{\Gamma}}}} \pi_{n / \xi_{\Gamma}}
$$

if $n \neq 0$. Thus we have computed the spectrum of $U$.

Theorem 4.1. We have $\Theta=\left\{\pi_{\alpha, \beta} \mid(\alpha, \beta) \in p(\Gamma)^{*}\right\} \cup\left\{\pi_{\gamma} \mid \gamma \in \xi_{\Gamma}^{-1} \mathbb{Z}, \gamma \neq 0\right\}$.

Our next aim is to compute the spectral multiplicities $m_{W}$ when $W \in \Theta_{\infty}$. For that we will apply Kirillov's orbit method [Ki] and the Howe-Richardson multiplicity formula (see $[\mathrm{Ho}],[\mathrm{Ri}]$ and an earlier work $[\mathrm{Mo}]$ ). In view of Proposition 1.3 we may assume without loss of generality that

$$
\Gamma=\left\{\left(\begin{array}{ccc}
1 & l & \frac{n}{k} \\
0 & 1 & m \\
0 & 0 & 1
\end{array}\right) \mid l, m, n \in \mathbb{Z}\right\}
$$

for some $k \in \mathbb{N}$ which is fixed. By Theorem 4.1, $\pi_{\gamma} \in \Theta$ if and only if $\gamma=k n$ with $n \in \mathbb{Z} \backslash\{0\}$. Thus our purpose is to calculate the multiplicity $m_{\pi_{k n}}$ for each $n \in \mathbb{Z} \backslash\{0\}$.

The Lie algebra of $H_{3}(\mathbb{R})$ is

$$
\mathfrak{h}_{3}(\mathbb{R}):=\left\{\left(\begin{array}{ccc}
0 & a & c \\
0 & 0 & b \\
0 & 0 & 0
\end{array}\right) \mid a, b, c \in \mathbb{R}\right\} .
$$

We endow it with the natural topology. Then the exponential map exp : $\mathfrak{h}_{3}(\mathbb{R}) \rightarrow$ $H_{3}(\mathbb{R})$ is a homeomorphism. We choose a special strong Malcev basis

$$
X_{1}:=\left(\begin{array}{ccc}
0 & 1 & 0 \\
0 & 0 & 0 \\
0 & 0 & 0
\end{array}\right), X_{2}:=\left(\begin{array}{ccc}
0 & 0 & 0 \\
0 & 0 & 1 \\
0 & 0 & 0
\end{array}\right), X_{3}:=\left(\begin{array}{ccc}
0 & 0 & \frac{1}{k} \\
0 & 0 & 0 \\
0 & 0 & 0
\end{array}\right)
$$

for $\mathfrak{h}_{3}(\mathbb{R})$. Fix an ideal $\mathfrak{m}:=\mathbb{R} X_{3}+\mathbb{R} X_{2}$ of $\mathfrak{h}_{3}(\mathbb{R})$. Then $\exp (\mathfrak{m})=H_{2, b}$. We consider the dual space $\mathfrak{h}_{3}(\mathbb{R})^{*}$ to $\mathfrak{h}_{3}(\mathbb{R})$ as the space of lower triangular $3 \times 3$ matrices (modulo the subspace of upper triangular matrices including the diagonal). The canonical duality form is given via the trace of the product, i.e.

$$
\mathfrak{h}_{3}(\mathbb{R}) \times \mathfrak{h}_{3}(\mathbb{R})^{*} \ni\left(\left(\begin{array}{ccc}
0 & d_{1} & d_{3} \\
0 & 0 & d_{2} \\
0 & 0 & 0
\end{array}\right),\left(\begin{array}{ccc}
* & * & * \\
z_{1} & * & * \\
z_{3} & z_{2} & *
\end{array}\right)\right) \mapsto z_{1} d_{1}+z_{2} d_{2}+z_{3} d_{3} .
$$

We note that then

$$
X_{1}^{*}=\left(\begin{array}{ccc}
* & * & * \\
1 & * & * \\
0 & 0 & *
\end{array}\right), X_{2}^{*}=\left(\begin{array}{ccc}
* & * & * \\
0 & * & * \\
0 & 1 & * \\
15 & &
\end{array}\right), X_{3}^{*}=\left(\begin{array}{ccc}
* & * & * \\
0 & * & * \\
k & 0 & *
\end{array}\right)
$$


is the dual basis for $\mathfrak{h}_{3}(\mathbb{R})^{*}$. Then $\mathfrak{m}^{*}=\mathbb{R} X_{2}^{*}+\mathbb{R} X_{3}^{*} \subset \mathfrak{h}_{3}(\mathbb{R})^{*}$. It is not difficult now to compute explicitly the co-adjoint action $\mathrm{Ad}^{*}$ of $H_{3}(\mathbb{R})$ on $\mathfrak{h}_{3}(\mathbb{R})^{*}$ :

$$
\operatorname{Ad}^{*}\left(c\left(t_{3}\right) b\left(t_{2}\right) a\left(t_{1}\right)\right)\left(\begin{array}{ccc}
* & * & * \\
z_{1} & * & * \\
z_{3} & z_{2} & *
\end{array}\right)=\left(\begin{array}{ccc}
* & * & * \\
z_{1}+t_{2} z_{3} & * & * \\
z_{3} & z_{2}-t_{1} z_{3} & *
\end{array}\right) .
$$

Hence the $\operatorname{Ad}^{*}\left(H_{3}(\mathbb{R})\right)$-orbit $\Omega(l)$ of a linear functional $l=\left(\begin{array}{ccc}* & * & * \\ z_{1} & * & * \\ z_{3} & z_{2} & *\end{array}\right) \in \mathfrak{h}_{3}(\mathbb{R})^{*}$ with $z_{3} \neq 0$ is $l+\mathbb{R} X_{2}^{*}+\mathbb{R} X_{1}^{*}$. Recall that according to Kirillov's orbit method $[\mathrm{Ki}], \widehat{H_{3}(\mathbb{R})}$ is in a one-to-one correspondence with $\operatorname{Ad}^{*}\left(H_{3}(\mathbb{R})\right)$-orbits in $\mathfrak{h}_{3}(\mathbb{R})^{*} \cdot{ }^{10}$ More precisely, the irreducible unitary representation of $H_{3}(\mathbb{R})$ corresponding to $\Omega(l)$ is induced from the following 1-dimensional representation (character) $\tau$ of $\exp (\mathfrak{m})=H_{2, b}$ :

$$
\tau(\exp X):=e^{2 \pi i\langle X, l\rangle}, \quad X \in \mathfrak{m} .
$$

It is straightforward to verify that $\operatorname{Ind}_{H_{2, b}}^{H_{3}(\mathbb{R})} \tau$ of $H_{3}(\mathbb{R})$ is unitarily equivalent to $\pi_{z_{3}}$.

We now explain the Howe-Richardson formula for $m_{\pi_{k n}}$. For that we let

$$
\mathcal{W}:=\left\{l \in \mathbb{Z} X_{3}^{*}+\mathbb{Z} X_{2}^{*} \mid l \uparrow\left(\mathbb{R} X_{3}\right) \neq 0\right\} .
$$

To put it another way,

$$
\mathcal{W}=\left\{\left(\begin{array}{ccc}
* & * & * \\
0 & * & * \\
k n & m & *
\end{array}\right) \mid n, m \in \mathbb{Z}, n \neq 0\right\} .
$$

Given $l \in \mathfrak{m}^{*} \subset \mathfrak{h}_{3}(\mathbb{R})^{*}$ and $g \in H_{3}(\mathbb{R})$, we let $\operatorname{Ad}_{0}^{*}(g) l:=\operatorname{Ad}^{*}(g) l\left\lceil\mathfrak{m} \in \mathfrak{m}^{*}\right.$. We now see that if $\gamma=c\left(t_{3}\right) b\left(t_{2}\right) a\left(t_{1}\right) \in \Gamma$ then

$$
\operatorname{Ad}_{0}^{*}(\gamma)\left(\begin{array}{ccc}
* & * & * \\
0 & * & * \\
k n & m & *
\end{array}\right)=\left(\begin{array}{ccc}
* & * & * \\
0 & * & * \\
k n & m-t_{1} n k & *
\end{array}\right)
$$

Since $t_{1} \in \mathbb{Z}$, it follows that $\operatorname{Ad}_{0}^{*}$ is an action of $\Gamma$ on $\mathcal{W}$. By [Ri] and [Ho], $m_{\pi_{z_{3}}}$ equals the number of $\operatorname{Ad}_{0}^{*}(\Gamma)$-orbits in the intersection $\Omega(l) \cap \mathcal{W}$. Hence

$$
m_{\pi_{z_{3}}}= \begin{cases}|n| k & \text { if } z_{3}=k n, n \in \mathbb{Z} \backslash\{0\} \\ 0 & \text { otherwise. }\end{cases}
$$

We deduce from this formula, Proposition 1.3 and Theorem 4.1 the following theorem.

Theorem 4.2. Let $\Gamma$ be an arbitrary lattice in $H_{3}(\mathbb{R})$. Then

$$
m_{\pi_{\gamma}}= \begin{cases}|n| k_{\Gamma} & \text { if } \gamma=n \xi_{\Gamma}^{-1}, n \in \mathbb{Z} \backslash\{0\} \\ 0 & \text { otherwise. }\end{cases}
$$

Therefore

$$
U=\bigoplus_{(\alpha, \beta) \in p(\Gamma)^{*}} \pi_{\alpha, \beta} \oplus \bigoplus_{0 \neq n \in \mathbb{Z}} \bigoplus_{1}^{|n| k_{\Gamma}} \pi_{n \xi_{\Gamma}^{-1}}
$$

In the next statement we show that the homogeneous actions of the Heisenberg group on nilmanifolds are isospectral.

${ }^{10}$ The case $z=0$ corresponds to the family of 1 -dimensional representations. 
Corollary 4.3. Let $\Gamma$ and $\Gamma^{\prime}$ be two lattices in $H_{3}(\mathbb{R})$. Denote by $T$ and $T^{\prime}$ the corresponding measure preserving actions of $H_{3}(\mathbb{R})$ on the homogeneous spaces $H_{3}(\mathbb{R}) / \Gamma$ and $H_{3}(\mathbb{R}) / \Gamma^{\prime}$ respectively. The following are equivalent:

(i) $T$ and $T^{\prime}$ are isomorphic.

(ii) $p(\Gamma)=p\left(\Gamma^{\prime}\right)$ and $k_{\Gamma}=k_{\Gamma^{\prime}}$.

(iii) $p(\Gamma)=p\left(\Gamma^{\prime}\right)$ and $\xi_{\Gamma}=\xi_{\Gamma^{\prime}}$.

(iv) The Koopman representations of $H_{3}(\mathbb{R})$ generated by $T$ and $T^{\prime}$ are unitarily equivalent.

(v) $T$ and $T^{\prime}$ have the same spectrum (i.e. the same maximal spectral type).

Proof. It is well known (and easy to see) that $T$ and $T^{\prime}$ are isomorphic if and only if $\Gamma$ and $\Gamma^{\prime}$ are conjugate. Therefore (i) and (ii) are equivalent according to the second claim of Proposition 1.3. It follows from Remark 1.6 that (ii) is equivalent (iii). Of course, (i) implies (iv). It follows from Theorem 4.2 that (iv) implies (iii). The equivalence of (iv) and (v) follows also from Theorem 4.2.

\section{HeisenBERG ODOMETERS}

In this section we describe the spectral decomposition of Heisenberg odometers into irreducible components. A system of algebraic invariants (consisting of two countable Abelian groups with a relation between them) is associated to every $H_{3}(\mathbb{R})$-odometer. It is shown that this system of invariants is complete for a certain f-isomorphism relation (to be defined below) on the class of Heisenberg odometers. We also show that the f-isomorphism is weaker then the isomorphism, i.e. the class of $H_{3}(\mathbb{R})$-odometers is not isospectral.

Let $\Gamma_{1} \supset \Gamma_{2} \supset \cdots$ be a nested sequence of lattices in $H_{3}(\mathbb{R})$. Denote by $(X, \mu, T)$ the associated $H_{3}(\mathbb{R})$-odometer. Let $(Y, \nu)$ stand for the space of $\left(T_{c(t)}\right)_{t \in \mathbb{R}^{-} \text {-ergodic }}$ components and let $f: X \rightarrow Y$ stand for the corresponding projection. Then an $\mathbb{R}^{2}$-action $V=\left(V_{t_{1}, t_{2}}\right)_{\left(t_{1}, t_{2}\right) \in \mathbb{R}^{2}}$ is well defined by the formula $V_{t_{1}, t_{2}} f(x):=$ $f\left(T_{b\left(t_{2}\right) a\left(t_{1}\right)} x\right)$. We call it the underlying $\mathbb{R}^{2}$-odometer. ${ }^{11}$

Definition 5.1. We say that $T$ is non-degenerate if one of the following equivalent conditions is satisfied:

(i) The underlying $\mathbb{R}^{2}$-odometer is non-transitive.

(ii) The subgroup $\bigcup_{j=1}^{\infty} p\left(\Gamma_{j}\right)^{*}$ is not closed in $\mathbb{R}^{2}$.

(iii) The sequence $\left(p\left(\Gamma_{j}\right)\right)_{j=1}^{\infty}$ does not stabilize, i.e. for each $j>0$ there is $j_{1}>j$ such that $p\left(\Gamma_{j}\right) \neq p\left(\Gamma_{j_{1}}\right)$.

Theorem 5.2. Let $U$ stand for the Koopman unitary representation of $H_{3}(\mathbb{R})$ generated by a Heisenberg odometer $T$.

(i) If $T$ is non-degenerate then

$$
U=\bigoplus_{(\alpha, \beta) \in \bigcup_{j=1}^{\infty} p\left(\Gamma_{j}\right)^{*}} \pi_{\alpha, \beta} \oplus \bigoplus_{0 \neq \gamma \in \bigcup_{j=1}^{\infty} \xi_{\Gamma_{j}}^{-1} \mathbb{Z}} \bigoplus_{1}^{\infty} \pi_{\gamma} .
$$

(ii) If there is $l>0$ such that $p\left(\Gamma_{j}\right)=p\left(\Gamma_{l}\right)$ for all $j \geq l$ then

$$
U=\bigoplus_{(\alpha, \beta) \in p\left(\Gamma_{l}\right)^{*}} \pi_{\alpha, \beta} \oplus \bigoplus_{0 \neq \gamma \in \bigcup_{j=l}^{\infty} \xi_{\Gamma_{j}}^{-1} \mathbb{Z}} \bigoplus_{1}^{m(\gamma)} \pi_{\gamma}
$$

${ }^{11}$ It is the $\mathbb{R}^{2}$-odometer associated with the sequence $\left(p\left(\Gamma_{j}\right)\right)_{j=1}^{\infty}$ of lattices $p\left(\Gamma_{j}\right)$ in $\mathbb{R}^{2}$. 
where $m(\gamma):=|\gamma| \xi_{\Gamma_{j}} k_{\Gamma_{j}}$ for each $\gamma \in \xi_{\Gamma_{j}}^{-1} \mathbb{Z}, j \geq l$.

Proof. (i) Consider the following nested family

$$
L^{2}\left(H_{3}(\mathbb{R}) / \Gamma_{1}\right) \subset L^{2}\left(H_{3}(\mathbb{R}) / \Gamma_{2}\right) \subset \cdots
$$

of $U$-invariant subspaces in $L^{2}(X)$. Their union $\bigcup_{j=1}^{\infty} L^{2}\left(H_{3}(\mathbb{R}) / \Gamma_{j}\right)$ is dense in $L^{2}(X)$. Therefore Theorem 4.2 yields that

$$
U=\lim _{j \rightarrow \infty}\left(\bigoplus_{(\alpha, \beta) \in p\left(\Gamma_{j}\right)^{*}} \pi_{\alpha, \beta} \oplus \bigoplus_{0 \neq n \in \mathbb{Z}} \bigoplus_{1}^{|n| k_{\Gamma_{j}}} \pi_{n \xi_{\Gamma_{j}}^{-1}}\right) .
$$

Thus we see that $U$ decomposes into a direct sum of 1-dimensional and infinite dimensional irreducible unitary representations. Of course, the multiplicity of every 1-dimensional irreducible sub-representation $\pi_{\alpha, \beta}$ of $U$ is 1 . Our purpose now is to compute the multiplicity of each infinite dimensional irreducible sub-representation $\pi_{\gamma}, 0 \neq \gamma \in \bigcup_{j=1}^{\infty} \xi_{\Gamma_{j}}^{-1} \mathbb{Z}$, of $U$. There are $j \in \mathbb{N}$ and $n \in \mathbb{Z} \backslash\{0\}$ such that

$$
\gamma=\frac{n}{\xi_{\Gamma_{j}}}=\frac{n q}{\xi_{\Gamma_{j+1}}}, \text { where } q:=\frac{\xi_{\Gamma_{j+1}}}{\xi_{\Gamma_{j}}}
$$

Then the multiplicity of $\pi_{\gamma}$ in $U \uparrow L^{2}\left(H_{3}(\mathbb{R}) / \Gamma_{j}\right)$ is $|n| k_{\Gamma_{j}}$ and the multiplicity of $\pi_{\gamma}$ in $U \uparrow L^{2}\left(H_{3}(\mathbb{R}) / \Gamma_{j+1}\right)$ is $|n| q k_{\Gamma_{j+1}}$. Let $p\left(\Gamma_{j}\right)=A_{j}\left(\mathbb{Z}^{2}\right)$ for some matrix $A_{j} \in G L_{2}(\mathbb{R}), j \in \mathbb{N}$. It follows from Remark 1.6 that

$$
\frac{k_{\Gamma_{j+1}}}{k_{\Gamma_{j}}}=\frac{\left|\operatorname{det} A_{j+1}\right|}{\left|\operatorname{det} A_{j}\right|} \cdot \frac{\xi_{\Gamma_{j}}}{\xi_{\Gamma_{j+1}}}
$$

Then

$$
|n| q k_{\Gamma_{j+1}}=|n| \frac{\xi_{\Gamma_{j+1}}}{\xi_{\Gamma_{j}}} k_{\Gamma_{j}} \frac{\left|\operatorname{det} A_{j+1}\right|}{\left|\operatorname{det} A_{j}\right|} \cdot \frac{\xi_{\Gamma_{j}}}{\xi_{\Gamma_{j+1}}}=|n| k_{\Gamma_{j}} \frac{\left|\operatorname{det} A_{j+1}\right|}{\left|\operatorname{det} A_{j}\right|} .
$$

Since $T$ is non-degenerated, we may assume without loss of generality that $p\left(\Gamma_{j}\right) \neq$ $p\left(\Gamma_{j+1}\right)$ and hence $\frac{\left|\operatorname{det} A_{j+1}\right|}{\left|\operatorname{det} A_{j}\right|} \geq 2$ for each $j$. Hence by Theorem 4.2 , the multiplicity of $\pi_{\gamma}$ in $U \uparrow L^{2}\left(H_{3}(\mathbb{R}) / \Gamma_{j+1}\right)$ is at least two times greater than the multiplicity of $\pi_{\gamma}$ in $U \uparrow L^{2}\left(H_{3}(\mathbb{R}) / \Gamma_{j}\right)$. It follows that the multiplicity of $\pi_{\gamma}$ in $U$ is infinite.

(ii) is proved in a similar way. It is enough to note that

$$
\frac{k_{\Gamma_{j+1}}}{k_{\Gamma_{j}}}=\frac{\xi_{\Gamma_{j}}}{\xi_{\Gamma_{j+1}}}
$$

for all $j \geq l$ and hence $m(\gamma)$ is well defined.

Definition 5.3. A subgroup $S$ in $\mathbb{R}^{m}$ is off-rational if its closure $\bar{S}$ is co-compact in $\mathbb{R}^{m}$ and there are a subgroup $Q \subset \mathbb{Q}^{m}$ and a matrix $A \in G L_{m}(\mathbb{R})$ such that $S=A Q$.

We now associate to $S$ an off-rational subgroup $\tau(S)$ in $\mathbb{R}$. Since $S$ is offrational, there is a sequence of matrices $A_{j} \in G L_{m}(\mathbb{R}) \cap M_{m}(\mathbb{Z})$ such that $A_{1}^{-1} \mathbb{Z}^{m} \subset$ 
$A_{2}^{-1} \mathbb{Z}^{m} \subset \cdots$ and $\bigcup_{j=1}^{\infty} A_{j}^{-1} \mathbb{Z}^{m}=Q$ and hence $S=\bigcup_{j=1}^{\infty} A A_{j}^{-1} \mathbb{Z}^{m} \cdot{ }^{12}$ Consider now a sequence of subgroups

$$
\frac{\operatorname{det} A}{\operatorname{det} A_{1}} \mathbb{Z} \subset \frac{\operatorname{det} A}{\operatorname{det} A_{2}} \mathbb{Z} \subset \cdots
$$

in $\mathbb{R}$. Then $\tau(S):=\bigcup_{j=1}^{\infty} \frac{\operatorname{det} A}{\operatorname{det} A_{j}} \mathbb{Z}$ is a dense off-rational subgroup of $\mathbb{R}$. It is straightforward to verify that $\tau(S)$ does not depend on the choice of the sequence $\left(A_{j}\right)_{j=1}^{\infty}$.

Suppose we are given a sequence $\Gamma=\left(\Gamma_{j}\right)_{j=1}^{\infty}$ of lattices $\Gamma_{1} \supset \Gamma_{2} \supset \cdots$ in $H_{3}(\mathbb{R})$. Then $S_{\Gamma}:=\bigcup_{j=1}^{\infty} p\left(\Gamma_{j}\right)^{*}$ is an off-rational subgroup of $\mathbb{R}^{2}$ and $\xi_{\Gamma}:=\bigcup_{j=1}^{\infty} \xi_{\Gamma_{j}}^{-1} \mathbb{Z}$ is an off-rational subgroup in $\mathbb{R}$. If $\{c(t) \mid t \in \mathbb{R}\} \cap \bigcap_{j=1}^{\infty} \Gamma_{j}=\{1\}$ then $\xi_{\Gamma}$ is dense in $\mathbb{R}$. Thus, in view of Theorem 3.1 , if $T$ is free then $\xi_{\Gamma}$ is dense in $\mathbb{R}$.

Proposition 5.4. We have $\tau\left(S_{\Gamma}\right) \supset \xi_{\Gamma}$.

Proof. Let $p\left(\Gamma_{j}\right)=A_{j}\left(\mathbb{Z}^{2}\right)$ for some $A_{j} \in G L_{2}(\mathbb{R}), j \in \mathbb{N}$. Since $k_{\Gamma_{j}}=\frac{\left|\operatorname{det} A_{j}\right|}{\xi_{\Gamma_{j}}}$ by Remark 1.6, we obtain that $\frac{1}{\operatorname{det} A_{j}} \mathbb{Z} \supset \frac{1}{\xi_{\Gamma_{j}}} \mathbb{Z}$ for each $j$.

Proposition 5.5. Given an off-rational subgroup $S$ in $\mathbb{R}^{2}$ and an off-rational subgroup $\xi$ in $\mathbb{R}$ such that $\tau(S) \supset \xi$, there is a sequence $\Gamma$ of lattices $\Gamma_{1} \supset \Gamma_{2} \supset \cdots$ in $H_{3}(\mathbb{Z})$ such that $S_{\Gamma}=S$ and $\xi_{\Gamma}=\xi$. If $S$ is dense then $\bigcap_{j=1}^{\infty} p\left(\Gamma_{j}\right)=\{0\}$. If, in addition, $\xi$ is dense in $\mathbb{R}$ then $\bigcap_{j=1}^{\infty} \Gamma_{j}=\{1\}$.

Proof. Let us choose for each $j>0$, a matrix $A_{j} \in G L_{2}(\mathbb{R})$ with $\operatorname{det} A_{j}>0$ and a positive real $\zeta_{j}$ such that $\left(A_{1}^{*}\right)^{-1} \mathbb{Z}^{2} \subset\left(A_{2}^{*}\right)^{-1} \mathbb{Z}^{2} \subset \cdots, \bigcup_{j=1}^{\infty}\left(A_{j}^{*}\right)^{-1} \mathbb{Z}^{2}=S, \zeta_{1}^{-1} \mathbb{Z} \subset$ $\zeta_{2}^{-1} \mathbb{Z} \subset \cdots$ and $\bigcup_{j=1}^{\infty} \zeta_{j}^{-1} \mathbb{Z}=\xi$. We then have $A_{1} \mathbb{Z}^{2} \supset A_{2} \mathbb{Z}^{2} \supset \cdots$ and $\zeta_{1} \mathbb{Z} \supset$ $\zeta_{2} \mathbb{Z} \supset$.. Since $\tau(S) \supset \xi$, we can assume without loss of generality (passing to a subsequence of $\left(A_{j}\right)_{j=1}^{\infty}$ if necessary) that $\operatorname{det}\left(A_{j}^{*}\right)^{-1} \mid \zeta_{j}^{-1}$ or, equivalently, $\zeta_{j} \mid \operatorname{det} A_{j}$ for each $j$. We now let

$$
\Gamma_{j}:=\left\{c\left(t_{3}\right) b\left(t_{2}\right) a\left(t_{1}\right) \mid\left(t_{1}, t_{2}\right) \in A_{j}\left(\mathbb{Z}^{2}\right), t_{3} \in \zeta_{j} \mathbb{Z}\right\}
$$

Then $\Gamma_{j}$ is a lattice in $H_{3}(\mathbb{R})$ with $\xi_{\Gamma_{j}}=\zeta_{j}, p\left(\Gamma_{j}\right)=A_{j}\left(\mathbb{Z}^{2}\right)$ and $k_{\Gamma_{j}}=\operatorname{det} A_{j} / \zeta_{j}$. We let $\Gamma:=\left(\Gamma_{j}\right)_{j=1}^{\infty}$. Of course, $\Gamma_{1} \supset \Gamma_{2} \supset \cdots, \xi_{\Gamma}=\xi$ and $S_{\Gamma}=S$.

If $S$ is dense in $\mathbb{R}^{2}$, it follows that $\bigcap_{j=1}^{\infty} p\left(\Gamma_{j}\right)=\{0\}$. If $\xi$ is dense in $\mathbb{R}$, it follows that $\bigcap_{j=1}^{\infty} \zeta_{j} \mathbb{Z}=\{0\}$ and therefore $\{c(t) \mid t \in \mathbb{R}\} \cap \bigcap_{j=1}^{\infty} \Gamma_{j}=\{1\}$. It follows that $\bigcap_{j=1}^{\infty} \Gamma_{j}=\{1\}$.

Definition 5.6. Two $H_{3}(\mathbb{R})$-odometers $T$ and $T^{\prime}$ are called $f$-isomorphic if they are associated with some sequences $\left(\Gamma_{j}\right)_{j=1}^{\infty}$ and $\left(\Gamma_{j}^{\prime}\right)_{j=1}^{\infty}$ (respectively) of lattices in $H_{3}(\mathbb{R})$ such that $\Gamma_{j}$ and $\Gamma_{j}^{\prime}$ are conjugate in $H_{3}(\mathbb{R})$ for each $j$.

Theorem 5.7. Let $\Gamma=\left(\Gamma_{j}\right)_{j=1}^{\infty}$ and $\Gamma^{\prime}=\left(\Gamma_{j}^{\prime}\right)_{j=1}^{\infty}$ be two sequences of lattices in $H_{3}(\mathbb{R})$ such that $\Gamma_{1} \supset \Gamma_{2} \supset \cdots$ and $\Gamma_{1}^{\prime} \supset \Gamma_{2}^{\prime} \supset \cdots$. Let $T$ denote the odometer associated to $\Gamma$ and let $T^{\prime}$ denote the odometer associated to $\Gamma^{\prime}$. Then $T$ and $T^{\prime}$ are f-isomorphic if and only if $S_{\Gamma}=S_{\Gamma^{\prime}}$ and $\xi_{\Gamma}=\xi_{\Gamma^{\prime}}$.

${ }^{12}$ We note that $A_{j}$ and $A$ are defined up to right multiplication with a matrix from $G L_{m}(\mathbb{Z})$. 
Proof. Of course, if $T$ and $T^{\prime}$ are f-equivalent then $S_{\Gamma}=S_{\Gamma^{\prime}}$ and $\xi_{\Gamma}=\xi_{\Gamma^{\prime}}$. Let us prove the converse. We first set $j_{1}:=1$. Since $S_{\Gamma}=S_{\Gamma^{\prime}}$ and $\xi_{\Gamma}=\xi_{\Gamma^{\prime}}$, there are $l_{1}>1$ and $j_{2}>j_{1}$ such that $p\left(\Gamma_{j_{1}}\right)^{*} \subset p\left(\Gamma_{l_{1}}^{\prime}\right)^{*} \subset p\left(\Gamma_{j_{2}}\right)^{*}$ and $\xi_{\Gamma_{j_{1}}}^{-1} \mathbb{Z} \subset \xi_{\Gamma_{l_{1}}^{\prime}}^{-1} \mathbb{Z} \subset$ $\xi_{\Gamma_{j_{2}}}^{-1} \mathbb{Z}^{13}$ Hence $p\left(\Gamma_{j_{1}}\right) \supset p\left(\Gamma_{l_{1}}^{\prime}\right) \supset p\left(\Gamma_{j_{2}}\right), \xi_{\Gamma_{j_{1}}} \mid \xi_{\Gamma_{l_{1}}^{\prime}}$ and $\xi_{\Gamma_{l_{1}}} \mid \xi_{\Gamma_{j_{2}}}$. We now let $\widetilde{\Gamma}_{j_{1}}:=\Gamma_{j_{1}} \cap p^{-1}\left(p\left(\Gamma_{l_{1}}^{\prime}\right)\right)$. Then $\widetilde{\Gamma}_{j_{1}}$ is a lattice in $H_{3}(\mathbb{R}),{ }^{14} \widetilde{\Gamma}_{j_{1}} \supset \Gamma_{j_{2}}, p\left(\widetilde{\Gamma}_{j_{1}}\right)=p\left(\Gamma_{l_{1}}^{\prime}\right)$ and $\xi_{\widetilde{\Gamma}_{j_{1}}}=\xi_{\Gamma_{j_{1}}}$. According to Lemma 1.5 there is a lattice $\widehat{\Gamma}_{j_{1}}$ such that $\widetilde{\Gamma}_{j_{1}} \supset$ $\widehat{\Gamma}_{j_{1}} \supset \Gamma_{j_{2}}$ with $p\left(\widehat{\Gamma}_{j_{1}}\right)=p\left(\Gamma_{l_{1}}^{\prime}\right)$ and $\xi_{\widehat{\Gamma}_{j_{1}}}=\xi_{\Gamma_{l_{1}}^{\prime}}$. It follows from Proposition 1.3 and Remark 1.6 that $\widehat{\Gamma}_{j_{1}}=q_{1} \Gamma_{l_{1}}^{\prime} q_{1}^{-1}$ for some element $q_{1} \in H_{3}(\mathbb{R})$. In the next step we argue in a similar way to find integers $j_{3}>j_{2}$ and $l_{2}>l_{1}$, a lattice $\widehat{\Gamma}_{j_{2}} \subset H_{3}(\mathbb{R})$ and an element $q_{2} \in H_{3}(\mathbb{R})$ such that $\Gamma_{j_{2}} \supset \widehat{\Gamma}_{j_{2}} \supset \Gamma_{j_{3}}$ and $\widehat{\Gamma}_{j_{2}}=q_{2} \Gamma_{l_{2}}^{\prime} q_{2}^{-1}$. Continuing this infinitely many times we obtain a sequence $\left(\widehat{\Gamma}_{j_{s}}\right)_{s=1}^{\infty}$ of lattices in $H_{3}(\mathbb{R})$, a subsequence $\left(\Gamma_{l_{s}}^{\prime}\right)_{s=1}^{\infty}$ of $\Gamma^{\prime}$ and a sequence $\left(q_{s}\right)_{s=1}^{\infty}$ of elements in $H_{3}(\mathbb{R})$ such that

$$
\begin{aligned}
& \Gamma_{j_{1}} \supset \widehat{\Gamma}_{j_{1}} \supset \Gamma_{j_{2}} \supset \widehat{\Gamma}_{j_{2}} \supset \cdots \text { and } \\
& \widehat{\Gamma}_{j_{s}}=q_{s} \Gamma_{l_{s}}^{\prime} q_{s}^{-1} \text { with } q_{s} \in H_{3}(\mathbb{R}) \text { for each } s>1 .
\end{aligned}
$$

It follows from (5-1) that $T$ is isomorphic to the $H_{3}(\mathbb{R})$-odometer associated with $\left(\widehat{\Gamma}_{j_{s}}\right)_{s=1}^{\infty}$. Of course, $T^{\prime}$ is isomorphic to the $H_{3}(\mathbb{R})$-odometer associated with $\left(\Gamma_{l_{s}}^{\prime}\right)_{s=1}^{\infty}$. Hence $T$ and $T^{\prime}$ are f-isomorphic.

Corollary 5.8. The Heisenberg odometers $T$ and $T^{\prime}$ are f-isomorphic if and only if the Koopman unitary representations of $H_{3}(\mathbb{R})$ associated with them are unitarily equivalent.

Proof. Let $T$ be associated with a sequence $\Gamma$ of nested lattices in $H_{3}(\mathbb{R})$ and let $T^{\prime}$ be associated with a sequence $\Gamma^{\prime}$ of nested lattices in $H_{3}(\mathbb{R})$. It follows from Theorem 5.2 that if the unitary representations of $H_{3}(\mathbb{R})$ associated with $T$ and $T^{\prime}$ are unitarily equivalent then $S_{\Gamma}=S_{\Gamma^{\prime}}$ and $\xi_{\Gamma}=\xi_{\Gamma^{\prime}}$. Applying Theorem 5.7 we conclude that $T$ and $T^{\prime}$ are f-isomorphic. The converse is obvious.

We now consider Cartesian products of Heisenberg odometers. First of all we recall the Clebsch-Gordan decompositions for the tensor products of two irreducible unitary representations of $H_{3}(\mathbb{R})$ (see $[\mathrm{Ki}]$ ):

$$
\begin{aligned}
\pi_{\alpha, \beta} \otimes \pi_{\alpha^{\prime}, \beta^{\prime}} & =\pi_{\alpha+\alpha^{\prime}, \beta+\beta^{\prime}}, \quad \text { for all } \alpha, \beta, \alpha^{\prime}, \beta^{\prime}, \\
\pi_{\gamma} \otimes \pi_{\gamma^{\prime}} & =\bigoplus_{1}^{\infty} \pi_{\gamma+\gamma^{\prime}} \quad \text { whenever } \gamma \neq-\gamma^{\prime}, \\
\pi_{\gamma} \otimes \pi_{-\gamma} & =\int_{\mathbb{R}^{2}} \pi_{\alpha, \beta} d \alpha d \beta \quad \text { for all } \gamma \\
\pi_{\gamma} \otimes \pi_{\alpha, \beta} & =\pi_{\gamma} \text { for all } \alpha, \beta, \gamma .
\end{aligned}
$$

\footnotetext{
${ }^{13}$ This is true because all the groups $p\left(\Gamma_{j}\right)^{*}, p\left(\Gamma_{j}^{\prime}\right)^{*}, \xi_{\Gamma_{j}}^{-1} \mathbb{Z}, \xi_{\Gamma_{j}^{\prime}}^{-1} \mathbb{Z}, j \in \mathbb{N}$, are finitely generated.

${ }^{14} \Gamma_{j_{1}}$ and $\widetilde{\Gamma}_{j_{1}}^{\prime}$ have the same $p$-fibers over $p\left(\Gamma_{l_{1}}^{\prime}\right)$.
} 
Proposition 5.9. Let $T$ and $T^{\prime}$ be two Heisenberg odometers associated with the nested sequences of lattices $\Gamma=\left(\Gamma_{j}\right)_{j=1}^{\infty}$ and $\Gamma^{\prime}=\left(\Gamma_{j}^{\prime}\right)_{j=1}^{\infty}$ in $H_{3}(\mathbb{R})$ respectively. Then

(i) $T \times T^{\prime}$ is ergodic if and only if $S_{\Gamma} \cap S_{\Gamma^{\prime}}=\{0\}$.

(ii) $T \times T^{\prime}$ is ergodic and has discrete maximal spectral type if and only if $S_{\Gamma} \cap S_{\Gamma^{\prime}}=\{0\}$ and $\xi_{\Gamma} \cap \xi_{\Gamma^{\prime}}=\{0\}$. In this case the Koopman unitary representation $U_{T \times T^{\prime}}$ of $H_{3}(\mathbb{R})$ decomposes into irreducible representations as follows

$$
U_{T \times T^{\prime}}=\bigoplus_{(\alpha, \beta) \in S_{\Gamma}+S_{\Gamma^{\prime}}} \pi_{\alpha, \beta} \oplus \bigoplus_{0 \neq \gamma \in \xi_{\Gamma}+\xi_{\Gamma^{\prime}}} \bigoplus_{1}^{\infty} \pi_{\gamma} .
$$

(iii) $T \times T^{\prime}$ is not isomorphic to any Heisenberg odometer. ${ }^{15}$

Proof. (i) and (ii) follow immediately from the Clebsch-Gordan decompositions, Theorem 4.1 and the fact that the invariant vectors of the Koopman unitary representation of $H_{3}(\mathbb{R})$ form the subspace corresponding to the component $\pi_{0,0}$.

(iii) Suppose that $T \times T^{\prime}$ is a Heisenberg odometer. Then it is ergodic and has discrete maximal spectral type. Hence by (ii), $\xi_{\Gamma} \cap \xi_{\Gamma^{\prime}}=\{0\}$. This implies that the sum $\xi_{\Gamma}+\xi_{\Gamma^{\prime}}$ is not off-rational. ${ }^{16}$ On the other hand, by (5-2) and Theorem 4.1, $\xi_{\Gamma}+\xi_{\Gamma^{\prime}}=\xi_{\Gamma^{\prime \prime}}$ for a nested sequence $\Gamma^{\prime \prime}$ of lattices in $H_{3}(\mathbb{R})$ that determines the Heisenberg odometer $T \times T^{\prime}$. Since $\xi_{\Gamma^{\prime \prime}}$ is always off-rational, we get a contradiction.

Our next purpose is to show that Heisenberg odometers (even the normal ones) are not isospectral.

Let $T$ be the Heisenberg odometer associated with a decreasing sequence $\left(\Gamma_{j}\right)_{j+1}^{\infty}$ of lattices in $H_{3}(\mathbb{Z})$. Let $(X, \mu)$ be the space of $T$ and fix $x \in X$. Then $x=\left(g_{j} \Gamma_{j}\right)_{j \in \mathbb{Z}}$ with $g_{j} \Gamma_{j}=g_{j+1} \Gamma_{j}$ for all $j \in \mathbb{N}$. Of course, we have $g_{1} \Gamma_{1} g_{1}^{-1} \supset g_{2} \Gamma_{2} g_{2}^{-1} \supset \cdots$. Let $T^{(x)}$ denote the odometer associated with the sequence $\left(g_{j} \Gamma_{j} g_{j}^{-1}\right)_{j=1}^{\infty}$ of lattices in $H_{3}(\mathbb{R})$. Denote by $X^{(x)}$ the space of this odometer. Then the map

$$
i^{(x)}: X \ni\left(h_{1} \Gamma_{1}, h_{2} \Gamma_{2}, \ldots\right) \mapsto\left(h_{1} g_{1}^{-1} \cdot g_{1} \Gamma_{1} g_{1}^{-1}, h_{2} g_{2}^{-1} \cdot g_{2} \Gamma_{2} g_{2}^{-1}, \ldots\right) \in X^{(x)}
$$

is a topological conjugacy of $T$ with $T^{(x)}$ such that $i^{(x)} x=\left(g_{j} \Gamma_{j} g_{j}^{-1}\right)_{j \in \mathbb{N}} \in X^{(x)}$. Below we call $i^{(x)}$ a changing the origin in $X$.

Lemma 5.10. Let $T$ and $T^{\prime}$ be $H_{3}(\mathbb{R})$-odometers associated with the sequences $\left(\Gamma_{j}\right)_{j \in \mathbb{N}}$ and $\left(\Gamma_{j}\right)_{j \in \mathbb{N}}$ respectively. If $T^{\prime}$ is a factor (in the measure theoretic sense) of $T$ then there are sequences $\left(g_{j}\right)_{j \in \mathbb{N}}$ and $\left(g_{j}^{\prime}\right)_{j \in \mathbb{N}}$ in $H_{3}(\mathbb{R})$ with $g_{j} \Gamma_{j}=g_{j+1} \Gamma_{j}$ and $g_{j}^{\prime} \Gamma_{j}^{\prime}=g_{j+1}^{\prime} \Gamma_{j}^{\prime}$ for all $j \in \mathbb{N}$ such that for each $n>0$, there is $m>0$ with $g_{m} \Gamma_{m} g_{m}^{-1} \subset g_{n}^{\prime} \Gamma_{n}^{\prime}\left(g_{n}^{\prime}\right)^{-1}$.

Proof. Let $(X, \mu)$ and $\left(X^{\prime}, \mu^{\prime}\right)$ be the spaces of $T$ and $T^{\prime}$. Let $\phi: X \rightarrow X^{\prime}$ be the Borel factor map intertwining $T$ with $T^{\prime}$. Denote by $p_{n}$ the canonical projection of

${ }^{15}$ As follows from the proof below, $T \times T^{\prime}$ even is not spectrally equivalent to any Heisenberg odometer.

${ }^{16}$ Indeed, if $\theta_{1} Q_{1} \cap \theta_{2} Q_{2}=0$ for some non-zero reals $\theta_{1}, \theta_{2}$ and nontrivial subgroups $Q_{1}, Q_{2} \subset \mathbb{Q}$ then $\theta_{1} / \theta_{2} \notin \mathbb{Q}$. If, furthermore, $\theta_{1} Q_{1}+\theta_{2} Q_{2}=\theta_{3} Q_{3}$ for a non-zero $\theta_{3} \in \mathbb{R}$ and nontrivial subgroup $Q_{3} \subset \mathbb{Q}$ then we get that $\theta_{3} / \theta_{2}$ is rational and irrational at the same time. 
$X$ onto $H_{3}(\mathbb{R}) / \Gamma_{n}$ and denote by $q_{n}$ the canonical projection of $X^{\prime}$ onto $H_{3}(\mathbb{R}) / \Gamma_{n}^{\prime}$. By Lusin's theorem, for each $n \in \mathbb{N}$, there is a closed subset $X_{n} \subset X$ with $\mu\left(X_{n}\right)>$ $1-2^{-25 n}$ such that the map $q_{n} \circ \phi$ is continuous on $X_{n}$. For each $n$, let us fix three elements $\gamma_{n, 1}, \gamma_{n, 2}$ and $\gamma_{n, 3}$ that generate $\Gamma_{n}$. It follows from the pointwise ergodic theorem that for each $n \in \mathbb{N}$ and $i \in\{1,2,3\}$ there is a subset $Y_{n} \subset X_{n}$ of "large" measure such that for each $x \in Y_{n}$, there exists a limit

$$
\lim _{N \rightarrow \infty} \frac{\#\left\{j \in\{1, \ldots, N\} \mid \gamma_{n, i}^{j} x \in X_{n}\right\}}{N}>0.9 .
$$

The "large" means that the series $\sum_{n=1}^{\infty}\left(1-\mu\left(Y_{n}\right)\right)$ converges. Hence by the BorelCantelli lemma, the intersection $\bigcap_{n>M}\left(X_{n} \cap Y_{n}\right)$ is non-empty for some $M \in \mathbb{N}$. Without loss of generality we may assume that $M=1$. Changing the origin in $X$, i.e. replacing $T$ with an isomorphic odometer, we may assume that

$$
e:=\left(\Gamma_{1}, \Gamma_{2}, \ldots\right) \in \bigcap_{n>M}\left(X_{n} \cap Y_{n}\right) .
$$

In a similar way, changing the origin in $X^{\prime}$ we may assume that

$$
\phi(e)=e^{\prime}:=\left(\Gamma_{1}^{\prime}, \Gamma_{2}^{\prime}, \ldots\right) .
$$

Under these assumptions we will show that for each $n>0$ there is $m>0$ with $\Gamma_{m} \subset \Gamma_{n}^{\prime}$.

Given $n$, we fix a small open neighborhood $\widetilde{U}$ of $\Gamma_{n}^{\prime}$ in $H_{3}(\mathbb{Z}) / \Gamma_{n}^{\prime}$. Then $U:=$ $q_{n}^{-1}(\widetilde{U})$ is an open neighborhood of $e^{\prime}$ in $X^{\prime}$. Therefore there is an open neighborhood $V$ of $e$ in $X$ such that

$$
\phi^{-1}(U) \supset V \cap X_{n} \neq \emptyset .
$$

By definition of the topology on $X$, we may assume without loss of generality that there are $m>0$ and an open neighborhood $\widetilde{V}$ of $\Gamma_{m}$ in $H_{3}(\mathbb{R}) / \Gamma_{m}$ such that $V=p_{m}^{-1}(\widetilde{V})$. For each $j \in \mathbb{N}$ and $i \in\{1,2,3\}$, we have $\gamma_{m, i}^{j} \Gamma_{m}=\Gamma_{m}$. Hence $\gamma_{m, i}^{j} e \in V$. Let $J_{N}:=\left\{j \in\{1, \ldots, N\} \mid \gamma_{m, i}^{j} e \in X_{n}\right\}$. Then for each $j \in J_{N}$, we have $\gamma_{m, i}^{j} e \in V \cap X_{n}$. It follows now from (5-3) and the fact that $\phi$ is equivariant that

$$
\gamma_{m, i}^{j} e^{\prime}=\gamma_{m, i}^{j} \phi(e)=\phi\left(\gamma_{m, i}^{j} e\right) \in U .
$$

Hence $\gamma_{m, i}^{j} \Gamma_{n}^{\prime} \in \widetilde{U}$ for each $j \in J_{N}$. Since $\#\left(J_{N}\right) / N>0.9$ for all large $N$ and $\widetilde{U}$ is small, it is easy to deduce that $\gamma_{m, i} \in \Gamma_{n}^{\prime}$. Indeed, $d:=p\left(\gamma_{m, i}\right) p\left(\Gamma_{n}^{\prime}\right) \in \mathbb{R}^{2} / p\left(\Gamma_{n}^{\prime}\right)$. As usual, $p$ stands for the standard projection of $H_{3}(\mathbb{R})$ onto $\mathbb{R}^{2}$. Take a weak limit point $\kappa$ of the sequence $\frac{1}{N} \sum_{j=1}^{N} \delta_{\gamma_{m, i}^{j} \Gamma_{n}^{\prime}}$ of measures on $H_{3}(\mathbb{R}) / \Gamma_{n}^{\prime}$. The natural projection

$$
v: H_{3}(\mathbb{R}) / \Gamma_{n}^{\prime} \rightarrow H_{3}(\mathbb{R}) / p^{-1}\left(p\left(\Gamma_{n}^{\prime}\right)\right)=\mathbb{T}^{2}
$$

maps $\kappa$ to the limit of the sequence $\frac{1}{N} \sum_{j=1}^{N} \delta_{d^{j}}$. This limit is the Haar measure, say $\lambda_{A}$, of the compact monothetic subgroup $A$ of $\mathbb{T}^{2}$ generated by the element $d$. Since we assumed that $\widetilde{U}$ is small, the projection $v(\widetilde{U})$ is also a small (in diameter) neighborhood of the unit in $\mathbb{T}^{2}$. We thus obtain that $\lambda_{A}(v(\widetilde{U}))>0.8$. (Of course, 
we may assume without loss of generality that the boundary of $v(\widetilde{U})$ is $\lambda_{A}$-null.) This is only possible if $A$ is trivial, i.e. $\gamma_{m, i} \in c(t) \Gamma_{n}^{\prime}$ for some $t \in\left[0, \xi_{\Gamma_{n}^{\prime}}\right)$. Thus $\kappa$ is supported by the circle $\mathbb{T}=v^{-1}\left(p^{-1}\left(p\left(\Gamma_{n}^{\prime}\right)\right)\right.$ ), which is the fiber of the fibration $v$ over the unit of $\mathbb{T}^{2}$. Since the intersection of $\widetilde{U}$ with this fiber is small in diameter, we apply a similar reasoning as above to conclude that $t=0$.

Therefore $\Gamma_{m} \subset \Gamma_{n}^{\prime}$.

We now provide an example of f-isomorphic but non-isomorphic odometers.

Example 5.11(cf. [Li-Ug, Example 4.9]). Fix a sequence of natural numbers $k_{1}<k_{2}<\cdots$ such that $k_{1}=1$ and $k_{n}\left(k_{n}+1\right)=k_{n+1}$ for each $n$. Let

$$
\begin{aligned}
& \Gamma_{n}:=\left\{c\left(k_{n} j_{3}\right) b\left(k_{n} j_{2}\right) a\left(k_{n} j_{1}\right) \mid j_{1}, j_{2}, j_{3} \in \mathbb{Z}\right\} \text { and } \\
& \Gamma_{n}^{\prime}:=\left\{c\left(k_{n} j_{3}+j_{1}\right) b\left(k_{n} j_{2}\right) a\left(k_{n} j_{1}\right) \mid j_{1}, j_{2}, j_{3} \in \mathbb{Z}\right\}
\end{aligned}
$$

$n \in \mathbb{N}$. It is easy to see that $H_{3}(\mathbb{Z})=\Gamma_{1} \supset \Gamma_{2} \supset \cdots$ and $H_{3}(\mathbb{Z})=\Gamma_{1}^{\prime} \supset \Gamma_{2}^{\prime} \supset \cdots$ Moreover, $\Gamma_{n}$ and $\Gamma_{n}^{\prime}$ are both normal in $H_{3}(\mathbb{Z})$ for each $n \in \mathbb{N}$. Denote by $T$ and $T^{\prime}$ the $H_{3}(\mathbb{R})$-odometers associated with the sequences $\left(\Gamma_{n}\right)_{n=1}^{\infty}$ and $\left(\Gamma_{n}^{\prime}\right)_{n=1}^{\infty}$ respectively. They are normal. Since $b\left(1 / k_{n}\right) \Gamma_{n}^{\prime} b\left(-1 / k_{n}\right)=\Gamma_{n}$ for each $n \in \mathbb{N}$, we obtain that $T$ and $T^{\prime}$ are f-isomorphic. If they were isomorphic then by Lemma 5.10 , there is $h \in H_{3}(R)$ such that for each $n>0$, there exists $m>0$ with $\Gamma_{m}^{\prime} \subset h \Gamma_{n} h^{-1}$. However $\xi_{\Gamma_{m}^{\prime}}=1$ for each $m$ but $\xi_{h \Gamma_{n} h^{-1}} \rightarrow \infty$ as $n \rightarrow \infty$, a contradiction. We also examine certain "symmetry" property for $T$ and $T^{\prime}$. Recall that a measure preserving action $R$ of $H_{3}(\mathbb{R})$ is called symmetric [Da4] if $R$ is isomorphic to $R \circ \theta$, where $\theta$ denotes the flip in $H_{3}(\mathbb{R})$. Since $\theta\left(H_{3}(\mathbb{Z})\right)=H_{3}(\mathbb{Z})$, the symmetric $H_{3}(\mathbb{Z})$ actions are defined in a similar way. It is easy to see that $T$ is symmetric because $\theta\left(\Gamma_{n}\right)=\Gamma_{n}$ for each $j$. It is straightforward to verify that

$$
\theta\left(\Gamma_{n}^{\prime}\right)=a\left(-1 / k_{n}\right) \Gamma_{n} a\left(1 / k_{n}\right)=\left\{c\left(k_{n} j_{3}+j_{2}\right) b\left(k_{n} j_{2}\right) a\left(k_{n} j_{1}\right) \mid j_{1}, j_{2}, j_{3} \in \mathbb{Z}\right\}
$$

for each $n$. We now show that $T^{\prime}$ is asymmetric. If $T^{\prime}$ and $T^{\prime} \circ \theta$ were isomorphic then by Lemma 5.10, there is $h \in H_{3}(R)$ such that for each $n>0$, there exists $m>0$ with $\Gamma_{m}^{\prime} \subset h \theta\left(\Gamma_{n}^{\prime}\right) h^{-1}$. However it is easy to see that

$$
\inf \left\{t \in \mathbb{R} \mid c(t) a(r) \in \Gamma_{m}^{\prime} \text { for some } r \in \mathbb{R}^{\prime}\right\}=1
$$

while

$$
\inf \left\{t \in \mathbb{R} \mid c(t) a(r) \in \theta\left(\Gamma_{n}^{\prime}\right) \text { for some } r \in \mathbb{R}^{\prime}\right\} \rightarrow \infty \quad \text { as } n \rightarrow \infty
$$

a contradiction. Hence $T^{\prime}$ is not symmetric. We also note that $T^{\prime}$ is f-isomorphic to $T^{\prime} \circ \theta$ because $\theta\left(\Gamma_{n}^{\prime}\right)=a\left(-1 / k_{n}\right) b\left(1 / k_{n}\right) \Gamma_{n}^{\prime} b\left(-1 / k_{n}\right) a\left(1 / k_{n}\right)$. Hence the Koopman representations $U_{T^{\prime}}$ and $U_{T^{\prime}} \circ \theta$ are unitarily equivalent.

We conclude this section with a "coordinate-free" definition of Heisenberg odometers.

Proposition 5.12. Let $T$ be an ergodic action of $H_{3}(\mathbb{R})$ on a standard probability space $(X, \mathfrak{B}, \mu)$. Let $\mathfrak{F}_{1} \subset \mathfrak{F}_{2} \subset \cdots$ be a sequence of factors of $T$ such that the union $\bigcup_{j=1}^{\infty} \mathfrak{F}_{j}$ is dense in $\mathfrak{B}$ and the restriction $T \uparrow \mathfrak{F}_{j}$ is isomorphic to a transitive $H_{3}(\mathbb{R})$-action on the 3-dimensional torus. Then $T$ is a Heisenberg odometer. 
Proof. Since the dynamical system $T \nmid \mathfrak{F}_{j}$ is transitive, it is isomorphic to the homogeneous space $H_{3}(\mathbb{R}) / \Gamma_{j}$ for a co-compact subgroup $\Gamma_{j} \subset H_{3}(\mathbb{R})$. Since $H_{3}(\mathbb{R}) / \Gamma_{j}$ is isomorphic to the 3 -dimensional torus, $\Gamma_{j}$ is a lattice. Thus the dynamical system $(X, \mu, T)$ is the projective limit of the following sequence of $H_{3}(\mathbb{R})$-homogeneous spaces

$$
H_{3}(\mathbb{R}) / \Gamma_{1} \stackrel{q_{1}}{\longleftarrow} H_{3}(\mathbb{R}) / \Gamma_{2} \stackrel{q_{2}}{\longleftarrow} \cdots .
$$

Hence there is a sequence $\left(g_{n}\right)_{n=1}^{\infty}$ of elements in $H_{3}(\mathbb{R})$ such that $\Gamma_{j} \supset g_{j}^{-1} \Gamma_{j+1} g_{j}$ and $q_{j}\left(g \Gamma_{j+1}\right)=g g_{j} \Gamma_{j}$ for each $j$. We now let

$$
\Gamma_{j}^{\prime}:=g_{1}^{-1} g_{2}^{-1} \cdots g_{j-1}^{-1} \Gamma_{j} g_{j-1} \cdots g_{2} g_{1}
$$

and define an equivariant isomorphism $r_{j}: H_{3}(\mathbb{R}) / \Gamma_{j} \rightarrow H_{3}(\mathbb{R}) / \Gamma_{j}^{\prime}$ by setting $r_{j}\left(g \Gamma_{j}\right):=g g_{j-1} \cdots g_{2} g_{1} \Gamma_{j}^{\prime}$. Then we obtain a nested sequence $\Gamma_{1}^{\prime} \supset \Gamma_{2}^{\prime} \supset \cdots$ of lattices in $\mathrm{H}_{3}(\mathbb{R})$. Moreover, the following infinite diagram commutes

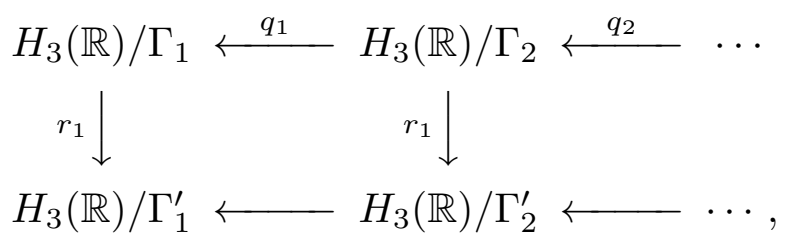

where the horizontal arrows in the bottom line denote the natural projections. Hence $T$ is isomorphic to the Heisenberg odometer associated with the sequence $\left(\Gamma_{j}^{\prime}\right)_{j=1}^{\infty}$.

\section{Joining StruCture of Heisenberg odometers}

In this section we describe the 2-fold ergodic self-joinings, as dynamical systems, for all Heisenberg odometers. We recall that a 2 -fold self-joining of a $G$-action $T=\left(T_{g}\right)_{g \in G}$ on a standard probability space $(X, \mathfrak{B}, \mu)$ is a measure $\lambda$ on the product space $(X \times X, \mathfrak{B} \otimes \mathfrak{B})$ such that $\lambda$ is invariant under the diagonal action $\left(T_{g} \times T_{g}\right)_{g \in G}$ and the projections of $\lambda$ to each of the two coordinates are $\mu$. The corresponding dynamical system $\left(X \times X, \lambda,\left(T_{g} \times T_{g}\right)_{g \in G}\right)$ is also called a 2-fold self-joining of $T$. A 2-fold self joining of $T$ is called off-diagonal if it is supported on the graph of a transformation of $(X, \mu)$ commuting with $T$. In this section we show that 2-fold self-joinings of Heisenberg odometers need not be Heisenberg odometers themselves, even in the transitive case. In particular, there is an ergodic nontransitive 2-fold self-joining of a transitive Heisenberg odometer. This is in sharp contrast with the actions with pure point spectrum. Indeed, each transitive action $T$ of a locally compact second countable group $G$ with pure point spectrum can be represented as an action by rotations (via an onto group homomorphism from $G$ to $K$ ) on a homogeneous space $K / H$, where $K$ is a compact second countable group and $H$ is a subgroup of $K$ [Ma3]. It is a standard argument (see [dJRu] for example) that each ergodic 2-fold self-joining of $T$ considered as a dynamical system is a factor of an ergodic 2-fold self-joining of the transitive $G$-action by rotations on $K$. All such self-joinings are off-diagonal, isomorphic to the original $G$-action on $K$, and hence transitive themselves. Since each factor of a transitive system is transitive, it follows that each ergodic 2-fold self-joining of a transitive action with pure point spectrum is transitive. Similar reasoning shows that the 
family of $G$-actions with pure point spectrum is also closed under the operation of taking ergodic 2-fold self-joinings. ${ }^{17}$

(A) Self-joinings of transitive Heisenberg odometers. Let $\Gamma$ be a lattice in $H_{3}(\mathbb{R})$. In view of Proposition 1.3 , we may assume without loss of generality that $\Gamma=\{c(n / k) b(m) a(l) \mid n, m, l \in \mathbb{Z}\}$ for some $k \in \mathbb{N}$. Every element $g \in H_{3}(\mathbb{R})$ can be written uniquely as $g=c\left(t_{3}\right) b\left(t_{2}\right) a\left(t_{1}\right) \gamma$ for some $\gamma \in \Gamma$ and $0 \leq t_{3}<1 / k$, $0 \leq t_{2}<1$ and $0 \leq t_{1}<1$. Then the quotient space $H_{3}(\mathbb{R}) / \Gamma$ is a 3 -torus

$$
\mathbb{T}^{3}=\left\{\left(t_{1}, t_{2}, t_{3}\right) \mid 0 \leq t_{1}<1,0 \leq t_{2}<1 \text { and } 0 \leq t_{3}<1 / k\right\} .
$$

Our purpose now is to describe the ergodic 2-fold self-joinings of the quotient (transitive) action $T=\left(T_{g}\right)_{g \in H_{3}(\mathbb{R})}$ of $H_{3}(\mathbb{R})$ on this space. We write it in skew product form as follows:

$$
T_{g}(y, z)=(p(g) \cdot y, \alpha(g, y)+z)
$$

where $(y, z) \in Y \times Z:=(\mathbb{R} / \mathbb{Z})^{2} \times\left(\mathbb{R} / k^{-1} \mathbb{Z}\right)$, the symbol "." denotes the usual action of $\mathbb{R}^{2}$ on $Y$ by rotations and $\alpha: H_{3}(\mathbb{R}) \times Y \rightarrow Z$ is the corresponding cocycle. Let $\widetilde{\Gamma}:=p^{-1}(p(\Gamma))=\{c(t) b(m) a(n) \mid t \in \mathbb{R}, n, m \in \mathbb{Z}\}$. Since $Y=H_{3}(\mathbb{R}) / \widetilde{\Gamma}$, the map $s: Y \ni\left(y_{1}, y_{2}\right) \mapsto b\left(y_{2}\right) a\left(y_{1}\right) \in H_{3}(\mathbb{R}), 0 \leq y_{1}, y_{2}<1$, is a cross-section of the quotient map $H_{3}(\mathbb{R}) \rightarrow H_{3}(\mathbb{R}) / \widetilde{\Gamma}$. Denote by $h_{s}: H_{3}(\mathbb{R}) \times Y \rightarrow \widetilde{\Gamma}$ the corresponding choice-cocycle. A direct calculation shows that

$$
\begin{aligned}
& h_{s}\left(c\left(t_{3}\right), y\right)=c\left(t_{3}\right), \\
& h_{s}\left(b\left(t_{2}\right), y\right)=b\left(\left[t_{2}+y_{2}\right]\right) c\left(-y_{1}\left[t_{2}+y_{2}\right]\right), \\
& h_{s}\left(a\left(t_{1}\right), y\right)=a\left(\left[t_{1}+y_{1}\right]\right) c\left(t_{1} y_{2}\right)
\end{aligned}
$$

for all $t_{1}, t_{2}, t_{3} \in \mathbb{R}$ and $y=\left(y_{1}, y_{2}\right), 0 \leq y_{1}, y_{2}<1$, where "[.]" denotes the integer part. By Remark 2.0 (in which $N=\widetilde{\Gamma}$ ), we may assume without loss of generality that $\alpha=\phi \circ h_{s}$ for a group homomorphism $\phi: \widetilde{\Gamma} \rightarrow Z$. We now compute $\phi$. For $\widetilde{\gamma}=c(t) b(m) a(n)$ with $t \in \mathbb{R}$ and $n, m \in \mathbb{Z}$, we have $T_{\widetilde{\gamma}} \Gamma=c(t) \Gamma$. Applying (6-1), we obtain

$$
T_{\widetilde{\gamma}}(\widetilde{\Gamma}, 0)=(\widetilde{\Gamma}, \alpha(\widetilde{\gamma}, \widetilde{\Gamma}))=\left(\widetilde{\Gamma}, t+k^{-1} \mathbb{Z}\right) .
$$

Thus, we obtain that $\phi(c(t) b(m) a(n))=t+k^{-1} \mathbb{Z}$. This yields

$$
\begin{aligned}
& \alpha\left(c\left(t_{3}\right), y\right)=t_{3}+k^{-1} \mathbb{Z}, \\
& \alpha\left(b\left(t_{2}\right), y\right)=-y_{1}\left[t_{2}+y_{2}\right]+k^{-1} \mathbb{Z}, \\
& \alpha\left(a\left(t_{1}\right), y\right)=t_{1} y_{2}+k^{-1} \mathbb{Z}
\end{aligned}
$$

for all $t_{1}, t_{2}, t_{3} \in \mathbb{R}$ and $y=\left(y_{1}, y_{2}\right), 0 \leq y_{1}, y_{2}<1$. For $d=\left(d_{1}, d_{2}\right) \in Y$, $0 \leq d_{1}, d_{2}<1$, we denote by $\alpha \circ d$ the cocycle

$$
H_{3}(\mathbb{R}) \times Y \ni(g, z) \mapsto \alpha \circ d(g, y):=\alpha(g, d+y) \in Z .
$$

${ }^{17}$ For each $G$-action $T$ with pure point spectrum there are a compact group $K$, a closed subgroup $H \subset K$ and a continuous homomorphism $\phi: G \rightarrow K$ with dense range in $K$ such that $T_{g} k H=\phi(g) k H$ for all $g \in G$ and $k \in K$ [Ma3]. Hence, by [dJRu], every ergodic 2-fold self-joining of $T$ is a factor of an ergodic 2 -fold self-joining of the $G$-action by rotations (via $\phi$ ) on $K$. The latter joining is off-diagonal; it is isomorphic as a dynamical system to the $G$-action by rotations (via $\phi$ ) on $K$. It remains to note that a factor of each action with pure point spectrum is an action with pure point spectrum. 
By Remark 2.0, we can represent the cocycle

$$
\alpha \times \alpha \circ d: H_{3}(\mathbb{R}) \times Y \rightarrow Z \times Z
$$

as $\psi \circ h_{s}$, where $\psi$ is a continuous group homomorphism from $\widetilde{\Gamma}$ to $Z \times Z$. To compute $\psi$, we first note that

$$
h_{s} \circ d(g, y)=h_{s}(g, y+d)=s(p(g) \cdot(y+d))^{-1} g s(y+d) .
$$

Substituting $y:=\widetilde{\Gamma}$ and $g:=c(t) b(m) a(n)$ we obtain that

$$
h_{s} \circ d(g, \widetilde{\Gamma})=s(d+\widetilde{\Gamma})^{-1} g s(d+\widetilde{\Gamma})=c\left(n d_{2}-m d_{1}\right) g .
$$

This formula, the formula for $\phi$ and Remark 2.0 yield that

$$
\psi(c(t) b(m) a(n))=\left(t+k^{-1} \mathbb{Z}, t+n d_{2}-m d_{1}+k^{-1} \mathbb{Z}\right) .
$$

If $d$ is aperiodic, i.e. $d_{1}$ or $d_{2}$ is irrational, then the subgroup $\psi(\widetilde{\Gamma})$ is dense in $Z \times Z$. It now follows from Remark 2.1 the essential range $E(\alpha \times \alpha \circ d)$ of the cocycle $\alpha \times \alpha \circ d$ is $Z \times Z$. We note however that $\psi(\widetilde{\Gamma}) \neq Z \times Z$. On the other hand, if $d$ is periodic, i.e. $d_{1}$ and $d_{2}$ are both rational, then there is a positive integer $q=q(d)$ such that

$$
E(\alpha \times \alpha \circ d)=\psi(\widetilde{\Gamma})=\bigcup_{j=0}^{q-1}\left\{\left(t+k^{-1} \mathbb{Z}, t+j /(q k)+k^{-1} \mathbb{Z} \mid 0 \leq t<1\right\} .\right.
$$

We denote this subgroup of $Z \times Z$ by $D_{q}$.

Let $\Delta_{d}$ denote the measure on $Y \times Y$ sitting on the subset $\{(y, d+y) \mid y \in Y\}$ and projecting on the Haar measure on $Y$ along each of the two coordinate projections. Given a closed subgroup $\Lambda$ in $Z \times Z$, we denote by $\lambda_{\Lambda}$ the Haar measure on $\Lambda$. We consider it as a measure on $Z \times Z$. Given $z \in Z$, we denote by $\lambda_{\Lambda} \circ z$ the image of $\lambda_{\Lambda}$ under the rotation $Z \times Z \ni\left(z_{1}, z_{2}\right) \mapsto\left(z_{1}, z_{2}+z\right) \in Z \times Z$.

Proposition 6.1. The set $J_{2}^{e}(T)$ of all ergodic 2 -fold self-joinings of $T$ is the union of two families as follows: ${ }^{18}$

$$
J_{2}^{e}(T)=\left\{\Delta_{d} \times \lambda_{Z \times Z} \mid d \text { is aperiodic }\right\} \cup\left\{\Delta_{d} \times \lambda_{D_{q(d)}} \circ z \mid d \text { is periodic, } z \in Z\right\} .
$$

Every joining from the first family is a non-transitive dynamical system and every joining from the second family is a transitive dynamical system.

Proof. Take $\kappa \in J_{2}^{e}(T)$. Represent $T$ in the skew product form (6-1). Then $\kappa$ is a measure on the space $Y \times Z \times Y \times Z$. Project $\kappa$ onto $Y \times Y$. The projection is an ergodic 2-fold self-joining on the transitive action of the 2-torus $Y$ on itself. Hence there is $d \in Y$ such that this projection is $\Delta_{d}$. It is a standard argument that the dynamical system $\left(\left(T_{g} \times T_{g}\right)_{g \in H_{3}(\mathbb{R})}, \kappa\right)$ can now be represented as the skew product over the same (as $T$ ) base $Y$ but with another cocycle $\alpha \times \alpha \circ d$. It remains to apply the argument preceding the statement of Proposition 6.1 to obtain the first assertion of the proposition.

Let $V$ denote the action of $\widetilde{\Gamma}$ on the compact group $\overline{\psi(\widetilde{\Gamma})}$ by translations via $\psi$, i.e. an element $\gamma \in \widetilde{\Gamma}$ acts as the translation by $\psi(\gamma)$. It is easy to see that the action $\left(\left(T_{g} \times T_{g}\right)_{g \in H_{3}(\mathbb{R})}, \kappa\right)$ is isomorphic to $\operatorname{Ind}_{\widetilde{\Gamma}}^{H_{3}(\mathbb{R})}(V)$, the second claim of the proposition follows from the fact whether or not $\psi(\widetilde{\Gamma})$ is closed and the following lemma.

${ }^{18}$ Provided that a special representation of $T$ as a skew product is chosen as above. 
Lemma 6.2. Let $G$ be a locally compact second countable group and let $H$ be a closed subgroup of $G$. A measure preserving action $V$ of $H$ is transitive if and only if the induced measure preserving action $\operatorname{Ind}_{H}^{G}(V)$ of $G$ is transitive.

Proof. If $V$ is non-transitive then, obviously, $\operatorname{Ind}_{H}^{G}(V)$ is non-transitive. Suppose now that $V$ is transitive. Denote by $N$ a closed subgroup of $H$ such that $V$ is isomorphic to the natural $H$-action on the homogeneous space $H / N$. Then $V$ is isomorphic to the induced action $\operatorname{Ind}_{N}^{H}(I)$, where $I$ denotes the trivial action of $N$ on a singleton. We now have (see [Ma1]) that

$$
\operatorname{Ind}_{H}^{G}(V)=\operatorname{Ind}_{H}^{G}\left(\operatorname{Ind}_{N}^{H}(I)\right)=\operatorname{Ind}_{N}^{G}(I),
$$

i.e. $\operatorname{Ind}_{H}^{G}(V)$ is isomorphic to the natural $G$-action on $G / N$.

Remark 6.3. We note that if an action of an Abelian locally compact group is transitive then every ergodic 2-fold self-joining of this action is also transitive (and isomorphic to the original action). As follows from Proposition 6.1, this is no longer true for the transitive actions of the Heisenberg group.

We are going to show now that an ergodic 2-fold self-joining of a transitive odometer can be rather "far from" the Heisenberg odometers. Though its maximal spectral type is discrete, the subgroup of 1-dimensional components of the spectrum is not off-rational.

Example 6.4. Consider a particular case when $k=1$, i.e. $\Gamma=H_{3}(\mathbb{Z})$. Suppose that $d_{1}$ is irrational (see (6-2)). Then the action $R=\left(R_{g}\right)_{g \in H_{3}(\mathbb{R})}$ of $H_{3}(\mathbb{R})$ defined on $Y \times Z \times Z$ by

$$
R_{g}(y, z):=\left(p(g) \cdot y, z+\psi\left(h_{s}(g, y)\right)\right), \quad y \in Y, z \in Z \times Z,
$$

is an ergodic 2-fold self-joining of $T$. Denote by $U_{R}$ the Koopman unitary representations of $H_{3}(\mathbb{R})$ generated by $R$. We are going to decompose $U_{R}$ into irreducible components. It follows from (6-4) that

$$
L^{2}\left(H_{3}(\mathbb{R}) / \Gamma\right)=\bigoplus_{n, m=1}^{\infty} L^{2}(Y) \otimes \eta_{n} \otimes \eta_{m}
$$

and each subspace $L^{2}(Y) \otimes \eta_{n} \otimes \eta_{m}$ is $U_{R}$-invariant, where $\eta_{n}(t):=\exp (2 \pi i n t)$, $t \in[0,1), n \in \mathbb{Z}$. Since

$$
R_{c(t)}(y, z)=\left(y, z+(t, t)+\mathbb{Z}^{2}\right) \quad \text { for all } t \in \mathbb{R},
$$

It follows that $U_{R}(c(t)) \uparrow\left(L^{2}(Y) \otimes \eta_{n} \otimes \eta_{m}\right)=\exp (2 \pi i t(n+m))$ Id. This yields

$$
U_{R}=\bigoplus_{\gamma \in \mathbb{Z}} \bigoplus_{r=1}^{\infty} \pi_{\gamma} \oplus \bigoplus_{k \in \mathbb{Z}} U_{R} \uparrow\left(L^{2}(Y) \otimes \eta_{k} \otimes \eta_{-k}\right)
$$

We note that the space $\mathcal{H}_{1}:=\bigoplus_{k \in \mathbb{Z}} L^{2}(Y) \otimes \eta_{k} \otimes \eta_{-k}$ is the subspace of vectors fixed by $\left(U_{R}(c(t))\right)_{t \in \mathbb{R}}$. It is straightforward to verify that $\mathcal{H}_{1}=L^{2}(Y) \otimes \mathcal{T}$, where

$$
\mathcal{T}:=\left\{v \in L^{2}(Z \times Z) \mid v\left(z_{1}, z_{2}\right)=v\left(z_{1}+z, z_{2}+z\right) \text { at a.a. } z_{1}, z_{2} \text { for each } z \in Z\right\}
$$


Hence $\mathcal{T}=L^{2}(\mathfrak{F})$, where $\mathfrak{F}$ is the sub- $\sigma$-algebra of sets in $Z \times Z$ measurable with respect to the map $Z \times Z \ni\left(z_{1}, z_{2}\right) \mapsto z_{1}-z_{2} \in Z$. Thus, the $\sigma$-algebra $\mathfrak{B}_{Y} \otimes \mathfrak{F}$ is a factor of $R$ such that $L^{2}\left(\mathfrak{B}_{Y} \otimes \mathfrak{F}\right)=\mathcal{H}_{1}$. In view of (6-4), the restriction $\widehat{R}=\left(\widehat{R}_{g}\right)_{g \in G}$ of $R$ to this factor can be written as

$$
\widehat{R}_{g}(y, z)=\left(p(g) \cdot y, z+\widetilde{\psi}\left(h_{s}(g, y)\right)\right),
$$

where $\widetilde{\psi}: \widetilde{\gamma} \rightarrow Z$ is the difference of the first and the second coordinate of $\widetilde{\psi}$, i.e., by $(6-2), \widetilde{\psi}(c(t) b(m) a(n))=m d_{1}-n d_{2}+\mathbb{Z}, t \in \mathbb{R}, n, m \in \mathbb{R}$. This means that $\widehat{R}$ is induced from the the following action of $\widetilde{\Gamma}$ on $Z$ by rotations

$$
\widetilde{\Gamma} \times Z \ni(c(t) b(m) a(n), z) \mapsto z+m d_{1}-n d_{2}+\mathbb{Z} .
$$

This action has a pure point spectrum and hence $\widehat{R}$ has pure point spectrum. It is straightforward to verify that

$$
U_{R} \uparrow \mathcal{H}_{1}=\bigoplus_{\alpha, \beta \in \Omega_{1}} \pi_{\alpha, \beta}
$$

where $\Omega_{1}=\mathbb{Z}^{2}+\left(\begin{array}{cc}d_{2} & 0 \\ 0 & -d_{1}\end{array}\right) \mathbb{Z}^{2}$. Since $d_{1}$ is irrational, $\Omega_{1}$ is not off-rational. In view of $(6-5)$,

$$
U_{R}=\bigoplus_{\gamma \in \mathbb{Z}} \bigoplus_{r=1}^{\infty} \pi_{\gamma} \oplus \bigoplus_{\alpha, \beta \in \Omega_{1}} \pi_{\alpha, \beta}
$$

Since $\Omega_{1}$ is not off-rational, $R$ is not isomorphic to any Heisenberg odometer. ${ }^{19}$

(B) Self-joinings of general Heisenberg odometers. We now consider a Heisenberg odometer $T$ associated to a sequence of latices $\Gamma_{1} \supset \Gamma_{2} \supset \cdots$ in $H_{3}(\mathbb{R})$. Then every ergodic 2-fold self-joining of $T$ is the inverse limit of a sequence of ergodic 2-fold self-joinings of the transitive actions of $H_{3}(\mathbb{R})$ on the homogeneous spaces $H_{3}(\mathbb{R}) / \Gamma_{j}, j \in \mathbb{N}$, viewed as factors of $T$. Using this simple observation and Proposition 6.1 we can explicitly describe all ergodic 2-fold self-joinings of $T$.

The $T$-action can be represented as a skew product. The space of this action is the product $Y \times Z$ of two compact Abelian groups $Y:=\operatorname{proj} \lim _{j \rightarrow \infty} \mathbb{R}^{2} / p\left(\Gamma_{j}\right)$ and $Z:=\operatorname{proj} \lim _{j \rightarrow \infty} Z_{j}$, where $Z_{j}:=\mathbb{R} / \xi_{\Gamma_{j}} \mathbb{Z}$. The factor-space $Y$ is the factor of $T$ generated by all proper functions of $T$; the restriction of $T$ to this factor has pure point spectrum. The corresponding cocycle of this factor with values in $Z$ is ergodic. ${ }^{20}$ Given $d \in Y$, we denote by $\Delta_{d}$ the image of the Haar measure on $Y$ under the map $Y \ni y \mapsto(y, y+d) \in Y \times Y$. Every element $d \in Y$ is a sequence $\left(d_{j}\right)_{j \in \mathbb{N}}$ of elements $d_{j} \in \mathbb{R}^{2} / p\left(\Gamma_{j}\right)$ such that $d_{j+1}$ maps to $d_{j}$ under the natural projection $\mathbb{R}^{2} / p\left(\Gamma_{j+1}\right) \rightarrow \mathbb{R}^{2} / p\left(\Gamma_{j}\right)$ for each $j$. In a similar way, every element $z \in Z$ is a sequence $\left(z_{j}\right)_{j \in \mathbb{N}}$ of elements $z_{j} \in Z_{j}$ such that $z_{j+1}$ maps to $z_{j}$ under the natural projection $Z_{j+1} \rightarrow Z_{j}$ for each $j$. If $d_{j}$ is periodic then we denote by $D_{j}$ be the closed subgroup of $Z_{j} \times Z_{j}$ associated with $d_{j}$ in the way described in the part (A) of this section (see (6-3)). We note that $D_{j}$ contains the diagonal of $Z_{j} \times Z_{j}$

\footnotetext{
${ }^{19}$ In fact, we have shown that $R$ is not spectrally equivalent to any Heisenberg odometer.

${ }^{20}$ See Section 7 for details.
} 
as a subgroup of finite index. Moreover, $D_{j+1}$ maps onto $D_{j}$ under the natural projection $Z_{j+1} \rightarrow Z_{j}$ for each $j$. Hence a projective limit $D_{d}:=\operatorname{proj} \lim _{j \rightarrow \infty} Z_{j}$ is well defined. It is a closed subgroup of $Z$. As in Proposition 6.1 we will use the following notation. Given a closed subgroup $\Lambda$ of $Z \times Z$, let $\lambda_{\Lambda}$ stand for the Haar measure on $\Lambda$. Given $z \in Z$, let $\lambda_{\Lambda} \circ z$ denote the image of $\lambda_{\Lambda}$ viewed as a measure on $Z \times Z$ under the rotation $\left(z_{1}, z_{2}\right) \mapsto\left(z_{1}, z_{2}+z\right)$ of $Z \times Z$.

Theorem 6.5. The set $J_{2}^{e}(T)$ of all ergodic 2-fold self-joinings of $T$ is the union of the following two families: ${ }^{21}$

$$
\begin{aligned}
J_{2}^{e}(T)= & \left\{\Delta_{d} \times \lambda_{Z \times Z} \mid d=\left(d_{j}\right)_{j \in \mathbb{N}} \text { with } d_{j} \text { aperiodic for each } j\right\} \\
& \cup\left\{\Delta_{d} \times \lambda_{D_{d}} \circ z \mid d=\left(d_{j}\right)_{j \in \mathbb{N}} \text { with } d_{j} \text { periodic for each } j \text { and } z \in Z\right\} .
\end{aligned}
$$

\section{On SpeCtral Determinacy of Heisenberg odometers}

(A) The case of transitive odometers. Let $T$ be an ergodic action of $H_{3}(\mathbb{R})$ on a standard probability space $(X, \mu)$. Denote by $U_{T}$ the corresponding Koopman unitary representation of $H_{3}(\mathbb{R})$.

Theorem 7.1. If $U_{T}$ is unitarily equivalent to the Koopman unitary representation generated by the action $Q$ of $H_{3}(\mathbb{R})$ by translations on $H_{3}(\mathbb{R}) / \Gamma$ for a lattice $\Gamma$ in $H_{3}(\mathbb{R})$ then $T$ is isomorphic to $Q$.

Proof. Without loss of generality we may assume that there is $k \geq 1$ such that $\Gamma=\left\{c\left(i_{3} / k\right) b\left(i_{2}\right) a\left(i_{1}\right) \mid i_{1}, i_{2}, i_{3} \in \mathbb{Z}\right\}$. By Theorem 4.1,

$$
U_{T}=\bigoplus_{(\alpha, \beta) \in \mathbb{Z}^{2}} \pi_{\alpha, \beta} \oplus \bigoplus_{0 \neq n \in \mathbb{Z}} \bigoplus_{j=1}^{|n|} \pi_{n k}
$$

up to the unitary equivalence. Denote by $\mathcal{H}$ the subspace of $L^{2}(X, \mu)$ where the unitary representation $\bigoplus_{(\alpha, \beta) \in \mathbb{Z}^{2}} \pi_{\alpha, \beta}$ is defined. Since each unitary representation $\pi_{\alpha, \beta}$ is 1-dimensional, it follows from the classical von Neumann theory of Abelian actions with pure point spectrum that there is a measure preserving factor map $q:(X, \mu) \rightarrow\left(\mathbb{R}^{2} / \mathbb{Z}^{2}, \lambda_{\mathbb{R}^{2} / \mathbb{Z}^{2}}\right)$ intertwining, for each $g \in H_{3}(\mathbb{R})$, the transformation $T_{g}$ with the rotation on $\mathbb{R}^{2} / \mathbb{Z}^{2}$ by $p(g)$ and such that $\mathcal{H}=\left\{f \circ q \mid f \in L^{2}\left(\mathbb{R}^{2} / \mathbb{Z}^{2}\right)\right\}$. Since $\pi_{n k}(c(t)) v=e^{2 \pi i n k t} v$ for each vector $v$ of the Hilbert space where $\pi_{n k}$ is defined, it follows from (7-1) that $\mathcal{H}$ is the subspace of vectors fixed by the unitary 1-parameter group $\left\{U_{T}(c(t)) \mid t \in \mathbb{R}\right\}$. Therefore by the von Neumann mean ergodic theorem, $\frac{1}{2 N} \int_{-N}^{N} U_{T}(c(t)) d t$ converges strongly as $N \rightarrow \infty$ to the orthogonal projection to $\mathcal{H}$ which is the mathematical expectation to the sub- $\sigma$-aldebra of $\left\{T_{c(t)} \mid t \in \mathbb{R}\right\}$-invariant subsets. Thus $q$ is the $\left\{T_{c(t)} \mid t \in \mathbb{R}\right\}$-ergodic decomposition and $\left(\mathbb{R}^{2} / \mathbb{Z}^{2}, \lambda_{\mathbb{R}^{2} / \mathbb{Z}^{2}}\right)$ is the space of $\left\{T_{c(t)} \mid t \in \mathbb{R}\right\}$-ergodic components. We note that $U_{T}\left(c\left(k^{-1}\right)\right)=\mathrm{Id}$. Moreover, if $0<s<k^{-1}$ then the unitary operator $U_{T}(c(s))$ has no non-trivial fixed vectors in the orthocomplement to $\mathcal{H}$ in $L^{2}(X)$. Hence the restriction of $\left\{T_{c(t)} \mid t \in \mathbb{R}\right\}$ to almost every ergodic component is isomorphic to the periodic flow with the smallest period $k^{-1}$. Thus $(X, \mu)$ splits into the product

$$
(X, \mu)=\left(\mathbb{R}^{2} / \mathbb{Z}^{2}, \lambda_{\mathbb{R}^{2} / \mathbb{Z}^{2}}\right) \times\left(\mathbb{R} / k^{-1} \mathbb{Z}, \lambda_{\mathbb{R} / k^{-1} \mathbb{Z}}\right)
$$

${ }^{21}$ Provided that some special cocycle representing $T$ as a skew product over its maximal factor with pure point spectrum is chosen. 
such that $q(y, z)=y$ and

$$
T_{c(t)}(y, z)=(y, t+z)
$$

for a.a. $y \in \mathbb{R}^{2} / \mathbb{Z}^{2}$ and $z \in \mathbb{R} / k^{-1} \mathbb{Z}$. Thus there exists a Borel cocycle

$$
\delta: H_{3}(\mathbb{R}) \times \mathbb{R}^{2} / \mathbb{Z}^{2} \rightarrow \mathbb{R} / k^{-1} \mathbb{Z}
$$

such that

$$
T_{g}(y, z)=(p(g) \cdot y, \delta(g, y)+z) \quad \text { for a.a. }(y, z) \in X .
$$

It follows that $T$ is a transitive action on a 3-dimensional torus. Hence there is a lattice $\Gamma^{\prime}$ in $H_{3}(\mathbb{R})$ such that $T$ is isomorphic to a homogeneous $H_{3}(\mathbb{R})$-action on $H_{3}(\mathbb{R}) / \Gamma^{\prime}$. Since the 1 -dimensional spectrum of $T$ is $\left\{\pi_{\alpha, \beta} \mid(\alpha, \beta) \in \mathbb{Z}^{2}\right\}$ we deduce from Theorem 4.2 that $p\left(\Gamma^{\prime}\right)=\mathbb{Z}^{2}$. It follows from $(7-2)$ that $\xi_{\Gamma^{\prime}}=k^{-1}$. Thus $p(\Gamma)=p\left(\Gamma^{\prime}\right)$ and $\xi_{\Gamma}=\xi_{\Gamma^{\prime}}$. Hence by Corollary $4.3, \Gamma$ and $\Gamma^{\prime}$ are conjugate, i.e. $T$ and $Q$ are isomorphic.

Remark 7.2.

(1) In fact, a stronger claim has been proved: if the maximal spectral types of $T$ and $Q$ are equivalent then $T$ and $Q$ are isomorphic.

(2) As a byproduct, we have discovered the following interesting fact: let $T$ be a transitive Heisenberg odometer and let $U_{T}$ denote the corresponding Koopman unitary representation of $H_{3}(\mathbb{R})$. If $V$ is a unitary representation of $H_{3}(\mathbb{R})$ with the same maximal spectral type as $U_{T}$ but with a different multiplicity function, then $V$ is not unitarily equivalent to the Koopman unitary representation of $H_{3}(\mathbb{R})$ generated by any probability preserving action of $H_{3}(\mathbb{R})$.

(B) The general case. Here we prove the main result of this section: the $H_{3}(\mathbb{R})$-odometers are not spectrally determined.

Lemma 7.3. Let $\xi=\bigcup_{j=1}^{\infty} d_{j}^{-1} \mathbb{Z}$ be an off-rational subgroup of $\mathbb{R}$ and let $S=$ $\bigcup_{j=1}^{\infty} A_{j}^{-1} \mathbb{Z}^{2}$ be an off-rational subgroup of $\mathbb{R}^{2}$. We set $Z:=\operatorname{proj} \lim _{j \rightarrow \infty} \mathbb{R} / d_{j} \mathbb{Z}$ and $Y:=\operatorname{proj} \lim _{j \rightarrow \infty} \mathbb{R}^{2} / A_{j}^{*} \mathbb{Z}^{2}$. Denote by $\lambda_{Z}$ and $\lambda_{Y}$ the Haar measure on $Z$ and $Y$ respectively. Denote by $\bar{r}_{j}: Z \rightarrow \mathbb{R} / d_{j}^{-1} \mathbb{Z}$ and $\bar{q}_{j}: Y \rightarrow \mathbb{R}^{2} / A_{j}^{*} \mathbb{Z}^{2}$ the canonical projections. Let $\vartheta: \mathbb{R} \rightarrow Z$ and $\varphi: \mathbb{R}^{2} \rightarrow Y$ be continuous homomorphisms defined by $\bar{r}_{j}(\vartheta(t))=t+d_{j}^{-1} \mathbb{Z}$ and $\bar{q}_{j}(\varphi(c))=c+A_{j}^{*} \mathbb{Z}^{2}$ for all $t \in \mathbb{R}, c \in \mathbb{R}^{2}, j \in \mathbb{N}$. Let $T$ be an ergodic measure preserving action on a probability space $(X, \mu)$. Suppose that the associated Koopman unitary representation $U_{T}$ of $H_{3}(\mathbb{R})$ decomposes into irreducibles as

$$
U_{T}=\bigoplus_{(\alpha, \beta) \in S} \pi_{\alpha, \beta} \oplus \bigoplus_{\gamma \in \xi} \bigoplus_{l=1}^{m_{\gamma}} \pi_{\gamma}
$$

for a spectral multiplicity map $m: \xi \ni \gamma \mapsto m_{\gamma} \in \mathbb{N} \cup\{\infty\}$. Then $(X, \mu)$ is isomorphic to the product $\left(Y \times Z, \lambda_{Y} \times \lambda_{Z}\right)$ in such a way that

$$
T_{g}(y, z)=\left(D_{g} y, \alpha(g, y)+z\right)
$$


where

$$
D: H_{3}(\mathbb{R}) \times Y \ni(g, y) \mapsto D_{g} y:=\varphi(p(g))+y \in Y
$$

is an ergodic action of $H_{3}(\mathbb{R})$ on $Y$ with pure point spectrum and

$$
\alpha: H_{3}(\mathbb{R}) \times Y \ni(g, y) \mapsto \alpha(g, y) \in Z
$$

is an ergodic cocycle of $D$ such that $\alpha(c(t), y)=\vartheta(t)$ for all $t \in \mathbb{R}$ and $y \in Y$.

Proof. We have a decomposition of $L^{2}(X, \mu)$ corresponding to (7-4):

$$
L^{2}(X, \mu)=\bigoplus_{(\alpha, \beta) \in S} \mathcal{H}_{\alpha, \beta} \oplus \bigoplus_{\gamma \in \xi} \bigoplus_{l=1}^{m_{\gamma}} \mathcal{H}_{\gamma, l}
$$

We now define a unitary representation $W=(W(z))_{z \in Z}$ of $Z$ in $L^{2}(X, \mu)$ by setting

$$
W(z) v= \begin{cases}v & \text { if } v \in \mathcal{H}_{\alpha, \beta},(\alpha, \beta) \in S \text { and } \\ e^{2 \pi i \gamma \bar{r}_{j}(z)} v & \text { if } v \in \mathcal{H}_{\gamma, l}, \gamma \in \xi, 1 \leq l \leq m_{\gamma}\end{cases}
$$

We consider here $\bar{r}_{j}(z)$ as a real number from the segment $\left[0, d_{j}\right)$. Then $W$ is continuous in the weak operator topology. Moreover,

$$
W(\vartheta(t))=U_{T}(c(t)) \quad \text { for all } t \in \mathbb{R} .
$$

Since the group $\operatorname{Aut}(X, \mu)$ of all $\mu$-preserving transformations of $X$ is a closed subgroup the unitary group $\mathcal{U}\left(L^{2}(X, \mu)\right)$ and the subgroup $\vartheta(\mathbb{R})$ is dense in $Z$, it follows that $W(z) \in \operatorname{Aut}(X, \mu)$ for all $z \in Z$. Thus, $\{W(z) \mid z \in Z\}$ is a compact group of transformations commuting with the action $T$. Therefore there are a probability space $(Y, \nu)$ and an action $D=\left(D_{g}\right)_{g \in H_{3}(\mathbb{R})}$ of $H_{3}(\mathbb{R})$ on it such that $(X, \mu)$ is isomorphic to the product $\left(Y \times Z, \nu \times \lambda_{Z}\right)$ such that

$$
T_{g}(y, z)=\left(D_{g} y, \alpha(g, y)+z\right) \quad \text { and } \quad W\left(z^{\prime}\right)(y, z)=\left(y, z+z^{\prime}\right)
$$

for all $g \in H_{3}(\mathbb{R})$ and $z^{\prime} \in Z$, where $\alpha: H_{3}(\mathbb{R}) \times Y \rightarrow Z$ is a measurable cocycle of $D$ (see, e.g. [dJRu]). It follows from $(7-5)$ that $\alpha(c(t), y)=\vartheta(t)$ for all $t \in \mathbb{R}$. Since

$$
\begin{aligned}
\bigoplus_{(\alpha, \beta) \in S} \mathcal{H}_{\alpha, \beta} & =\left\{v \in L^{2}(X, \mu) \mid U_{T}(c(t)) v=v \text { for all } t \in \mathbb{R}\right\} \\
& =\left\{v \in L^{2}(X, \mu) \mid W(z) v=v \text { for all } z \in Z\right\}
\end{aligned}
$$

it follows that $L^{2}(Y)=\bigoplus_{(\alpha, \beta) \in S} \mathcal{H}_{\alpha, \beta}$ and the Koopman unitary representation of $H_{3}(\mathbb{R})$ generated by $D$ decomposes into irreducible ones as $U_{D}=\bigoplus_{(\alpha, \beta) \in S} \pi_{\alpha, \beta}$. Therefore without loss of generality we may assume that $Y$ is a compact Abelian group dual to $S$ and $D_{g} y=\varphi(p(g))+y$ for all $y \in Y$ and $g \in H_{3}(\mathbb{R})$.

Now we prove a converse to Lemma 7.3. 
Lemma 7.4. Let $S, \xi, Y, Z$ and $D$ be as in Lemma 7.3.

(i) Given a cocycle $\beta$ of $D$ with values in $Z$ such that

$$
\beta(c(t), y)=\vartheta(t) \quad \text { for all } t \in \mathbb{R},
$$

there is a map $m: \xi \ni \gamma \mapsto m_{\gamma} \in \mathbb{N} \cup\{\infty\}$ such that the Koopman unitary representation $U_{D^{\beta}}$ of $H_{3}(\mathbb{R})$ generated by the $\beta$-skew product extension $D^{\beta}$ of $D$ is unitarily equivalent to (7-4).

(ii) Given two cocyles $\beta, \beta^{\prime}$ of $D$ with values in $Z$ satisfying (7-6), the skew product extensions $D^{\beta}$ and $D^{\beta^{\prime}}$ of $D$ are isomorphic if and only if there exist an element $y_{0} \in Y$ and a Borel map $f: Y \rightarrow Z$ such that

$$
\beta\left(g, y+y_{0}\right)=f(y)+\beta^{\prime}(g, y)-f(\varphi(p(g))+y)
$$

for each $g \in H_{3}(\mathbb{R})$ at a.a. $y \in Y$.

Proof. (i) We first note that $\xi$ is isomorphic to the dual $\widehat{Z}$ of $Z$. Hence we can identify an element $\gamma$ of $\xi$ with a character $\xi_{\gamma} \in L^{2}\left(Z, \lambda_{Z}\right)$. Then we obtain a decomposition of $L^{2}\left(Y \times Z, \lambda_{Y} \times \lambda_{Z}\right)$ into an orthogonal sum of $U_{D^{\beta} \text {-invariant }}$ subspaces:

$$
L^{2}\left(Y \times Z, \lambda_{Y} \times \lambda_{Z}\right)=\bigoplus_{\gamma \in \xi} L^{2}\left(Y, \lambda_{Y}\right) \otimes \xi_{\gamma}
$$

It follows from the property of $\beta$ that

$$
U_{D^{\beta}}(c(t))\left(v \otimes \xi_{\gamma}\right)=\xi_{\gamma}(\vartheta(t)) v \otimes \xi_{\gamma}=e^{2 \pi i \gamma t} v \otimes \xi_{\gamma}, \quad v \in L^{2}\left(Y, \lambda_{Y}\right) .
$$

Therefore $U_{D^{\beta}} \uparrow\left(L^{2}\left(Y, \lambda_{Y}\right) \otimes \xi_{\gamma}\right)=\bigoplus_{1}^{m_{\gamma}} \pi_{\gamma}$ for some multiplicity $m_{\gamma} \in \mathbb{N} \cup\{\infty\}$ for each $\gamma \in \xi, \gamma \neq 0$. Of course, $U_{D^{\beta}} \uparrow\left(L^{2}\left(Y, \lambda_{Y}\right) \otimes 1\right)=\bigoplus_{(\alpha, \beta) \in S} \mathcal{H}_{\alpha, \beta}$. It follows that (7-4) holds, as desired.

(ii) Since $D^{\beta}$ and $D^{\beta^{\prime}}$ are isomorphic, it follows from a rather standard description of the centralizer of an ergodic compact group extension of an action by rotations on a compact Abelian group (see, e.g., [Da1]) that there exist an element $y_{0} \in Y$, a continuous automorphism $\theta$ of $Z$ and a Borel map $f: Y \rightarrow Z$ such that

$$
\beta\left(g, y+y_{0}\right)=f(y)+\theta\left(\beta^{\prime}(g, y)\right)-f(\varphi(p(g))+y)
$$

for each $g \in H_{3}(\mathbb{R})$ at a.a. $y \in Y$. Now (7-6) yields that $\theta(\vartheta(t))=\vartheta(t)$ for all $t \in \mathbb{R}$. Since $\vartheta$ has a dense range in $Z$, it follows that $\theta$ is the identity, as desired.

Consider now the case where $T$ is the $H_{3}(\mathbb{R})$-odometer associated with a sequence $\Gamma_{1} \supset \Gamma_{2} \supset \cdots$ of lattices in $H_{3}(\mathbb{R})$. Denote by $X$ the space of $T$. We set $Y_{j}:=$ $\mathbb{R}^{2} / p\left(\Gamma_{j}\right)$ and $Z_{j}:=\mathbb{R} / \xi_{\Gamma_{j}} \mathbb{Z}$. Recall that the homogeneous space $H_{3}(\mathbb{R}) / \Gamma_{j}$ is isomorphic to the product $Y_{j} \times Z_{j}$ in such a way that the natural $H_{3}(\mathbb{R})$-action has a skew product form as follows

$$
g \cdot(y, z):=\left(p(g) \cdot y, \alpha_{j}(g, y)+z\right)
$$

for all $(y, z) \in Y_{j} \times Z_{j}$ and $g \in H_{3}(\mathbb{R})$, where $\alpha_{j}: H_{3}(\mathbb{R}) \times Y_{j} \rightarrow \mathbb{R} / \xi_{\Gamma_{j}} \mathbb{Z}$ is a cocycle satisfying

$$
\alpha_{j}(c(t), y)=t+\xi_{\Gamma_{j}} \mathbb{Z}
$$


The natural projection $H_{3}(\mathbb{R}) / \Gamma_{j} \rightarrow H_{3}(\mathbb{R}) / \Gamma_{j-1}$ corresponds to the mapping

$$
Y_{j} \times Z_{j} \ni(y, z) \mapsto\left(q_{j}(y), r_{j}\left(z+f_{j}(y)\right)\right) \in Y_{j-1} \times Z_{j-1}
$$

where $q_{j}: Y_{j} \rightarrow Y_{j-1}$ and $r_{j}: Z_{j} \rightarrow Z_{j-1}$ are the natural projections and $f_{j}$ : $Y_{j} \rightarrow Z_{j}$ is a Borel map. ${ }^{22}$ It is easy to see that the $H_{3}(\mathbb{R})$-equivariance of the map $H_{3}(\mathbb{R}) / \Gamma_{j} \rightarrow H_{3}(\mathbb{R}) / \Gamma_{j-1}$ is equivalent to the following property

$$
\alpha_{j-1}\left(g, q_{j}(y)\right)=r_{j}\left(f_{j}(p(g) \cdot y)+\alpha_{j}(g, y)-f_{j}(y)\right)
$$

for all $g \in G$ and $y \in Y_{j}$. Therefore, replacing $\alpha_{j}$ with a cohomologous cocycle, we may assume without loss of generality that

$$
\alpha_{j-1}\left(g, q_{j}(y)\right)=r_{j}\left(\alpha_{j}(g, y)\right)
$$

for every $j$. Of course, this cohomological change will not affect property (7-8). We now set $Y:=\operatorname{proj} \lim _{j \rightarrow \infty} Y_{j}$ and $Z:=\operatorname{proj} \lim _{j \rightarrow \infty} Z_{j}$ in the category of compact Abelian groups furnished with the Haar measures. Denote by $\bar{q}_{j}: Y \rightarrow Y_{j}$ and $\bar{r}_{j}: Z \rightarrow Z_{j}$ the canonical projections. We note that $Z$ and $Y$ are the same objects as in Lemmata 7.3 and 7.4 if we set $\xi:=\bigcup_{j=1}^{\infty} \xi_{\Gamma_{j}}^{-1} \mathbb{Z}$ and $S:=\bigcup_{j=1}^{\infty} p\left(\Gamma_{j}\right)^{*}$. Recall that $D$ is an ergodic action of $H_{3}(\mathbb{R})$ on $Y$ with pure point spectrum $S$ (see the statement of Lemma 7.3). In view of (7-9), a cocycle $\alpha: H_{3}(\mathbb{R}) \times Y \rightarrow Z$ of $D$ is well defined by

$$
\bar{r}_{j}(\alpha(g, y))=\alpha_{j}\left(g, \bar{q}_{j}(y)\right), \quad j \in \mathbb{N} .
$$

It follows from (7-8) that $\alpha(c(t), y)=\vartheta(t)$ for all $t \in \mathbb{R}$ and $y \in Y$. Since $X=$ proj $\lim _{j \rightarrow \infty} H_{3}(\mathbb{R}) / \Gamma_{j}$, we obtain that $X$ is measure theoretically isomorphic to $Y \times Z$ in such a way that $T$ is the $\alpha$-skew product extension $D^{\alpha}$ of $D$.

Definition 7.5. Let $Y, Z$ and $D$ be as above. A Borel cocycle $\alpha$ of $D$ with values in $Z$ is called finitary if there are cocycles $\alpha_{j}$ of the natural action of $H_{3}(\mathbb{R})$ on $Y_{j}$ with values in $Z_{j}$ such that (7-10) is satisfied.

Remark 7.6. For $y_{0} \in Y$, we define a $Z$-valued cocycle $\beta$ of $D$ by setting $\beta(g, y):=$ $\alpha\left(g, y+y_{0}\right)$. If $\alpha$ is finitary then so is $\beta$.

We now state one of the main results of this section.

Theorem 7.7. Let $S$ be an off-rational subgroup of $\mathbb{R}^{2}$ and let $\xi$ be an off-rational subgroup of $\mathbb{R}$ such that $\tau(S) \supset \xi$. Fix a nested sequence $\Gamma=\left(\Gamma_{j}\right)_{j=1}^{\infty}$ of lattices in $H_{3}(\mathbb{R})$ with $S_{\Gamma}=S$ and $\xi_{\Gamma}=\xi^{23}$. Let $T$ be an ergodic action of $H_{3}(\mathbb{R})$ on a standard probability space. Suppose that (7-4) holds for the associated Koopman unitary representation $U_{T}$ of $H_{3}(\mathbb{R})$. As in Lemma 7.3, represent $T$ as a skew product extension $D^{\alpha}$ of $D$ for a cocycle $\alpha$ of $D$ with values in $Z$. Then $T$ is an $H_{3}(\mathbb{R})$-odometer (which is f-isomorphic to the $H_{3}(\mathbb{R})$-odometer associated with $\Gamma$ ) if and only if $\alpha$ is cohomologous to a finitary cocycle.

${ }^{22}$ This follows from the fact that the projection $H_{3}(\mathbb{R}) / \Gamma_{j} \rightarrow H_{3}(\mathbb{R}) / \Gamma_{j-1}$ intertwines the actions of the center of $H_{3}(\mathbb{R})$ on these homogeneous spaces while $Y_{j}$ is the space of ergodic components for the action of the center.

${ }^{23}$ It exists by Proposition 5.5. 
Proof. $(\Rightarrow)$ If $T$ is a Heisenberg odometer then (as was shown above) there exists a representation $T$ as $D^{\alpha^{\prime}}$ with a finitary cocycle $\alpha^{\prime}$. By Lemma 7.4(ii), $\alpha$ is cohomologous to a 'rotation of $\alpha^{\prime}$ by an element of $Y$ '. As was noted in Remark 7.6, the class of finitary cocycles is invariant under such rotations. Thus, $\alpha$ is cohomologous to a finitary cocycle.

$(\Leftarrow)$ Conversely, suppose that $\alpha$ is finitary. Then a sequence of cocycles $\alpha_{j}$ : $H_{3}(\mathbb{R}) \times Y_{j} \rightarrow Z_{j}$ is well defined by (7-10). Since $\alpha(c(t), y)=\varphi(t)$ for all $t \in \mathbb{R}$ and $y \in Y$, it follows from (7-10) that $\alpha_{j}$ satisfies (7-8) for each $j \in \mathbb{N}$. This yields that the $\alpha_{j}$-skew product extension $H_{3}(\mathbb{R})$ (see $\left.(7-7)\right)$ is transitive on $Y_{j} \times Z_{j}$. Then by Proposition 5.12, $T$ is a Heisenberg odometer.

Corollary 7.8. Under the notation of Theorem 7.7, if $S$ is closed in $\mathbb{R}^{2}$ then $\mathrm{T}$ is an $\mathrm{H}_{3}(\mathbb{R})$-odometer. This means that the subclass of degenerate Heisenberg odometers is spectrally determined.

We now consider the problem of spectral determinacy within the entire class Heisenberg odometers.

Corollary 7.9. Let $T$ be a non-degenerate Heisenberg odometer associated with a sequence $\Gamma=\left(\Gamma_{j}\right)_{j=1}^{\infty}$ of lattices in $H_{3}(\mathbb{R})$. Then there is an ergodic action $R$ of $H_{3}(\mathbb{R})$ such that

- $R$ has the same maximal spectral type as $T$ but

- $R$ is not isomorphic to $T$ (and hence to any $H_{3}(\mathbb{R})$-odometer).

Proof. By Lemma 7.3 and Theorem 7.7, we can represent $T$ as $D^{\beta}$ for a $Z$-valued finitary cocycle $\beta$ of $D$ satisfying (7-6). Let $\alpha: \mathbb{R}^{2} \times Y \rightarrow Z$ be a cocycle of the underlying $\mathbb{R}^{2}$-odometer. By analogy with Definition 7.5 , we call $\alpha$ finitary if

$$
\bar{r}_{j}(\alpha(s, y))=\alpha_{j}\left(s, \bar{q}_{j}(y)\right), \quad s \in \mathbb{R}^{2}, j \in \mathbb{N}
$$

for a sequence of cocycles $\alpha_{j}: \mathbb{R}^{2} \times Y_{j} \rightarrow Z_{j}$. Since $T$ is non-degenerated, the underlying $\mathbb{R}^{2}$-odometer is not transitive. Therefore there is a cocycle $\alpha$ of it which is not cohomologous to a finitary one. Then we consider a $Z$-valued cocycle

$$
\beta^{\prime}(g, y):=\beta(g, y)+\alpha(p(g), y)
$$

of $D$. Of course, $\beta^{\prime}$ satisfies (7-6). Therefore by Lemma 7.4(i), the maximal spectral type of the $\beta^{\prime}$-skew product extension $D^{\beta^{\prime}}$ of $D$ coincides with the maximal spectral type of $T$. However $\beta^{\prime}$ is not finitary. ${ }^{24}$ Therefore by Theorem $7.7, D^{\beta^{\prime}}$ is not isomorphic to an odometer.

To construct an explicit counter-example to the spectral determinacy of nondegenerate Heisenberg odometers we first restate Theorem 7.7 in a more suitable (for this purpose) form. Let $\left(\Gamma_{j}\right)_{j=1}^{\infty}, D, Z$ and $\alpha$ be the same objects as in Lemma 7.3 and Theorem 7.7. We need some more notation. Let $D^{\prime}$ denote the $p\left(\Gamma_{1}\right)$-odometer associated with the sequence $p\left(\Gamma_{1}\right) \supset p\left(\Gamma_{2}\right) \supset \cdots$ of cofinite subgroups in $p\left(\Gamma_{1}\right)$. Let $Y^{\prime}:=\operatorname{proj} \lim _{j \rightarrow \infty} p\left(\Gamma_{1}\right) / p\left(\Gamma_{j}\right)$ be the space of $D^{\prime}$. We note that $Y^{\prime}$ is a compact Abelian group (furnished with Haar measure). Let $\widetilde{\Gamma}_{1}:=p^{-1}\left(p\left(\Gamma_{1}\right)\right)$. Denote by $\widetilde{D}=\left(\widetilde{D}_{\gamma}\right)_{\gamma \in \widetilde{\Gamma}_{1}}$ the following $\widetilde{\Gamma}_{1}$-action on $Y^{\prime}$ :

$$
\widetilde{D}_{\gamma} y^{\prime}:=p(\gamma) \cdot y^{\prime}
$$

${ }^{24}$ This is because $\beta$ is finitary and $\alpha$ is not finitary. 
Then one can deduce easily from Corollary 2.5 that $D=\operatorname{Ind}_{\widetilde{\Gamma}_{1}}^{H_{3}(\mathbb{R})}(\widetilde{D})$. Therefore by Lemma 2.4(iii), there is a $Z$-valued cocycle $\beta$ of $\widetilde{D}$ such that $\alpha$ is cohomologous to the induced cocycle $\widetilde{\beta}$ of $D$. Moreover, by Lemma 2.4(ii), the cohomology class of $\beta$ is defined uniquely by a cohomology class of $\alpha$. Let $\mathfrak{Y}_{j}$ denote the factor of $D$ generated by the canonical projection $\bar{p}_{j}: Y \rightarrow Y_{j}$ and let $\widetilde{\mathfrak{Y}}_{j}$ denote the factor of $\widetilde{D}$ generated by the canonical projection $\bar{p}_{j}^{\prime}: Y^{\prime} \rightarrow p\left(\Gamma_{1}\right) / p\left(\Gamma_{j}\right)$. It is straightforward to verify that $\mathfrak{Y}_{j}$ is induced by $\widetilde{\mathfrak{Y}}_{j}$. Therefore Lemma $2.4(\mathrm{ii})$, (iv) yield that $\alpha$ is cohomologous to a finitary cocycle if and only if $\beta$ is cohomologous to a finitary cocycle, say $\beta^{\prime}$, of $\widetilde{D}$, i.e. the map

$$
Y^{\prime} \ni y^{\prime} \mapsto \bar{r}_{j}\left(\beta^{\prime}\left(\gamma, y^{\prime}\right)\right) \in Z_{j}
$$

is $\widetilde{\mathfrak{Y}}_{j}$-measurable for each $\gamma \in \widetilde{\Gamma}_{1}$ and $j \in \mathbb{N}$. Thus we obtain the following corollary from Theorem 7.7 .

Corollary 7.10. Using the notation of Theorem 7.7, $T$ is an $H_{3}(\mathbb{R})$-odometer (associated with $\Gamma$ ) if and only if $\beta$ is cohomologous to a finitary cocycle.

We are now ready to construct an action of $H_{3}(\mathbb{R})$ which is unitarily equivalent to a Heisenberg odometer but not isomorphic to it.

Example 7.11. Let $p_{1}, p_{2}, \ldots$ be an infinite sequence of pairwise different primes, $p_{1}=1$. We set

$$
\Gamma_{j}:=\left\{c(n) b\left(p_{1} \cdots p_{j} m\right) a\left(p_{1} \cdots p_{j} l\right) \mid n, m, l \in \mathbb{Z}\right\} .
$$

Then $\Gamma:=\left(\Gamma_{j}\right)_{j=1}^{\infty}$ is a decreasing sequence of lattices in $H_{3}(\mathbb{Z})$. Denote by $T$ the associated $H_{3}(\mathbb{R})$-odometer. As in Lemma 7.3, represent $T$ as a skew product extension $T=D^{\alpha}$ for a cocycle $\alpha$ of $D$ with values in $Z$. We note that in this case $Z=Z_{j}=\mathbb{R} / \mathbb{Z}$ for each $j \in \mathbb{N}$. By Theorem 7.7, $\alpha$ is (up to a cohomological change) finitary. It is straightforward to verify that the following holds

(i) $Y^{\prime}=\operatorname{proj} \lim _{j \rightarrow \infty} \mathbb{Z}^{2} / p_{1} \cdots p_{j} \mathbb{Z}^{2}$,

(ii) $\widetilde{\Gamma}_{1}=\{c(t) b(m) a(l) \mid t \in \mathbb{R}, m, l \in \mathbb{Z}\}$ and

(iii) $\alpha$ is cohomologous to the cocycle induced from the cocycle $\beta$ of $\widetilde{D}$ given by $\beta\left(\gamma, y^{\prime}\right)=t+\mathbb{Z}$ if $\gamma=c(t) b(m) a(n) \in \widetilde{\Gamma}_{1}$.

Thus $\beta$ is finitary. It follows from (i) and the mutual coprimeness of $p_{j}, j \in \mathbb{N}$, that the dynamical system $\left(Y^{\prime}, D^{\prime}\right)$ is isomorphic to the infinite product $Y^{\prime}=$ $\bigotimes_{j \in \mathbb{N}} \mathbb{Z}^{2} / p_{j} \mathbb{Z}^{2}$ such that

$$
D_{\gamma}^{\prime}\left(y_{1}, y_{2}, \ldots\right)=\left(\gamma_{1}+y_{1}, \gamma_{2}+y_{2}, \ldots\right)
$$

for all $\gamma \in p\left(\Gamma_{1}\right)=\mathbb{Z}^{2}$ and $y_{j} \in \mathbb{Z}^{2} / p_{j} \mathbb{Z}^{2}$, where $\gamma_{j}:=\gamma+p_{j} \mathbb{Z}^{2}, j \in \mathbb{N}$. Partition $\mathbb{N}$ into two infinite disjoint subsets $J_{1}$ and $J_{2}$ and let $Y_{1}^{\prime}:=\bigotimes_{j \in J_{1}} \mathbb{Z}^{2} / p_{j} \mathbb{Z}^{2}$ and $Y_{2}^{\prime}:=\bigotimes_{j \in J_{2}} \mathbb{Z}^{2} / p_{j} \mathbb{Z}^{2}$. Then $Y_{1}^{\prime}$ and $Y_{2}^{\prime}$ are natural (coordinate) factors of $D^{\prime}$. Denote the restriction of $D^{\prime}$ to these factors by $D^{1}$ and $D^{2}$ respectively. We see that $D^{1}$ and $D^{2}$ are both free and $D^{\prime}$ is naturally isomorphic to the Cartesian product $D^{1} \times D^{2}$.

We now note that the group $Y^{\prime}$ acting on itself by rotations is the centralizer $C\left(D^{\prime}\right)$ of $D^{\prime}$. Since $D^{\prime}$ has pure point spectrum, $\widetilde{\mathfrak{Y}}_{j}$ is the $\sigma$-algebra of subsets fixed 
by a compact subgroup of $Y^{\prime}$. We can find a family of elements $\left(S_{j}\right)_{j \in \mathbb{N}}$ in $C\left(D^{\prime}\right)$ such that the following two properties are satisfied:

(iv) $\widetilde{\mathfrak{Y}}_{j}$ is fixed by $S_{j}$, i.e. $S_{j}$ is trivial on $\widetilde{\mathfrak{Y}}_{j}$.

(v) If we represent $S_{j}$ as the product $S_{j}=S_{1, j} \times S_{2, j}$ with $S_{1, j} \in C\left(D^{1}\right)$ and $S_{2, j} \in C\left(D^{2}\right)$ then the 'joint' action

$$
\mathbb{Z}^{2} \times \bigoplus_{j=1}^{\infty} \mathbb{Z} \ni\left(\gamma, n_{1}, n_{2}, \ldots\right) \mapsto D_{\gamma}^{1} S_{1,1}^{n_{1}} S_{1,2}^{n_{2}} \ldots
$$

of the group $\mathbb{Z}^{2} \times \bigoplus_{j=1}^{\infty} \mathbb{Z}$ on $Y_{1}^{\prime}$ is free.

By an auxiliary Lemma 7.12 (see the end of this section), there is a cocycle $\omega$ of $D^{1}$ with values in $Z$ such that the cocycle $\bar{\omega}:=\bigotimes_{j \in J_{1}} \omega \circ S_{1, j}$ with values in $Z^{J_{1}}$ is ergodic and, moreover, the product $\mathbb{Z}^{2}$-action $\left(D^{1}\right)^{\bar{\omega}} \times D^{2}$ on $Y_{1}^{\prime} \times Z^{J_{1}} \times Y_{2}$ is ergodic. In other words, the $Z^{J_{1}}$-valued cocycle $\bar{\omega} \otimes 1$ of $D^{\prime}$ (recall that $D^{\prime}=D^{1} \times D^{2}$ ) is ergodic.

Claim A. For any $j$, the cocycle $\omega \otimes 1$ of $D^{\prime}$ is not cohomologous to a $\mathfrak{Y}_{j^{-}}$ measurable cocycle. Indeed, if $\omega \otimes 1$ is cohomologous to a $\mathfrak{Y}_{j}$-measurable cocycle then in view of (iv), $\omega \otimes 1$ is cohomologous to the cocycle $(\omega \otimes 1) \circ S_{j}$. Therefore the $Z^{2}$-valued cocycle $\omega \otimes 1 \times(\omega \otimes 1) \circ S_{j}$ of $D^{\prime}$ is not ergodic. However it is easy to see that

$$
\omega \otimes 1 \times(\omega \otimes 1) \circ S_{j}=\left(\omega \times \omega \circ S_{1, j}\right) \otimes 1
$$

and the skew product $\left(D^{\prime}\right)^{\left(\omega \times \omega \circ S_{1, j}\right) \otimes 1}$ is a factor of the skew product $\left(D^{\prime}\right)^{\bar{\omega} \otimes 1}$ which is erdodic by the choice of $\omega$. We get a contradiction which proves Claim A.

We now define a $Z$-valued cocycle $\kappa$ of $\widetilde{D}$ by setting

$$
\kappa\left(\gamma, y^{\prime}\right):=\beta\left(\gamma, y^{\prime}\right)+(\omega \otimes 1)\left(p(\gamma), y^{\prime}\right)
$$

for each $\gamma \in \widetilde{\Gamma}_{1}$ and $y^{\prime} \in Y^{\prime}$. Denote by $\widetilde{\alpha}$ the $Z$-valued cocycle of $D$ induced from $\kappa$. Finally, we let $\widetilde{T}:=D^{\widetilde{\alpha}}$. Then $\widetilde{T}$ is an ergodic $H_{3}(\mathbb{R})$-action.

It follows from Claim $\mathrm{A}$ that $\kappa$ is not cohomologous to a finitary cocycle. Now Corollary 7.10 yields that $\widetilde{T}$ is not an $H_{3}(\mathbb{R})$-odometer. On the other hand, by Lemma 7.4(i), the maximal spectral type of the Koopman unitary representation $U_{\widetilde{T}}$ coincides with the maximal spectral type of $U_{T}$. Since $\xi_{\Gamma}=\mathbb{Z}$ and $\pi_{n}$ is contained in $U_{T}$ with infinite multiplicity for each $n \neq 0$ by Theorem 5.2(i), to prove that $T$ and $\widetilde{T}$ are unitarily equivalent it remains to show that the multiplicity $\pi_{n}$ in $U_{\widetilde{T}}$ is also infinite for each $n \neq 0$.

Denote by $U$ the Koopman unitary representation of $\widetilde{\Gamma}_{1}$ generated by the skew product $\widetilde{D}^{\kappa}$. The space of $U$ is $L^{2}\left(Y^{\prime} \times Z\right)$. It decomposes into an orthogonal sum

$$
L^{2}\left(Y^{\prime} \times Z\right)=\bigoplus_{\eta \in \widehat{Y_{2}^{\prime}}} \bigoplus_{n \in \mathbb{Z}} L^{2}\left(Y_{1}^{\prime}\right) \otimes \eta \otimes \chi_{n}
$$

of $U$-invariant subspaces $L^{2}\left(Y_{1}^{\prime}\right) \otimes \eta \otimes \chi_{n} \cdot{ }^{25}$ Since the Koopman unitary representation $U_{\widetilde{T}}$ is induced from $U$, we obtain the following decomposition of $L^{2}(Y \times Z)$

${ }^{25}$ The subspace $L^{2}\left(Y_{1}^{\prime}\right) \otimes \eta \otimes \chi_{n}$ is invariant under $U$ because $\omega \otimes 1$ as a function of $\left(\gamma, y^{\prime}\right)=$ $\left(\gamma, y_{1}^{\prime}, y_{2}^{\prime}\right) \in \widetilde{\Gamma}_{1} \times Y_{1}^{\prime} \times Y_{2}^{\prime}$ does not depend on $y_{2}^{\prime}$ while $\beta$ does not depend on $y^{\prime}$ at all. 
into $U_{\widetilde{T}}$-invariant subspaces:

$$
L^{2}(Y \times Z)=\bigoplus_{\eta \in \widehat{Y_{2}^{\prime}}} \bigoplus_{n \in \mathbb{Z}} L^{2}\left(\mathbb{R}^{2} / \mathbb{Z}^{2}\right) \otimes L^{2}\left(Y_{1}^{\prime}\right) \otimes \eta \otimes \chi_{n}
$$

Moreover, it is straightforward to verify that $U_{\widetilde{T}}(c(t)) v=e^{2 \pi i n t} v$ for each vector $v \in L^{2}\left(\mathbb{R}^{2} / \mathbb{Z}^{2}\right) \otimes L^{2}\left(Y_{1}^{\prime}\right) \otimes \eta \otimes \chi_{n}$. Therefore the restriction of $U_{\widetilde{T}}$ to the subspace $L^{2}\left(\mathbb{R}^{2} / \mathbb{Z}^{2}\right) \otimes L^{2}\left(Y_{1}^{\prime}\right) \otimes \eta \otimes \chi_{n}$ is a multiple of $\pi_{n}$ for each $n \neq 0$ and $\eta \in \widehat{Y_{2}^{\prime}}$. Since the group $\widehat{Y_{2}^{\prime}}$ is infinite, the multiplicity of $\pi_{n}$ in $U_{\widetilde{T}}$ is also infinite, as desired.

We now state and prove an auxiliary lemma used in Example 7.11.

Lemma 7.12. Let $K$ be a compact metric group. Let $R=\left(R_{g}\right)_{g \in G}$ and $Q=$ $\left(Q_{g}\right)_{g \in G}$ be two ergodic actions of an infinite countable discrete Abelian group $G$ such that $R$ is free and the Cartesian product $R \times Q$ is ergodic. Fix a countable infinite Abelian subgroup $A$ in the centralizer $C(R)$ of $R$ such that $A \cap\left\{R_{g} \mid g \in\right.$ $G\}=\{\mathrm{Id}\}$. Then there is a cocycle $\omega$ of $R$ with values in $K$ such that the $K^{A_{-}}$ valued cocycle $\bar{\omega}:=\bigotimes_{a \in A} \omega \circ$ a of $R$ is ergodic and the Cartesian product $R^{\bar{\omega}} \times Q$ is ergodic.

Proof. To prove this lemma we will use the orbit theory of amenable actions and their cocycles (see $[\mathrm{Sc}],[\mathrm{BeG}],[\mathrm{GoSi}]$, [Da1]). Let $(X, \mu)$ denote the space of $R$. We denote by $\mathcal{R} \subset X \times X$ the $R$-orbit equivalence relation. Recall that the full group $[\mathcal{R}]$ of $\mathcal{R}$ is the group of all $\mu$-preserving invertible transformations $F$ of $X$ such that $F x$ belongs to the $\mathcal{R}$-class of $x$ for a.a. $x \in X$. Choose

(i) a weakly mixing free action $V=\left(V_{g}\right)_{g \in G}$ of $G$ such that the orbit equivalence relation of $V$ is $\mathcal{R}$ and

(ii) a cocycle $\tau$ of $V$ with values in $K$ such that the skew product $V^{\tau}$ is weakly mixing.

Let $V^{A}=\left(V^{A}(g)\right)_{g \in G}$ denote the product $G$-action on the product space $\left(X^{A}, \mu^{A}\right)$, i.e. $\left(V_{g}^{A} x_{b}\right):=V_{g} x_{b}$ for each $x=\left(x_{b}\right)_{b \in A} \in X^{A}$ and $g \in G$. We consider also a shift action $S=\left(S_{a}\right)_{a \in A}$ of $A$ on $X^{A}$ :

$$
\left(S_{a} x\right)_{b}:=x_{b-a}, \quad a, b \in A, \quad x=\left(x_{b}\right)_{b \in A} \in X^{A} .
$$

Of course,

(iii) $S_{a} \in C\left(V^{A}\right)$ and $S_{a} \notin\left\{V_{b}^{A} \mid b \in A\right\}$ for each $a \in A \backslash\{0\}$.

We define a cocycle $\tau^{A}$ of $V^{A}$ with values in $K$ by setting $\tau^{A}(g, x):=\tau\left(g, x_{0}\right)$ for all $g \in G$ and $x \in X^{A}$. Consider the following cocycle $\overline{\tau^{A}}$ of $V^{A}$ with values in $K^{A}$ :

$$
\overline{\tau^{A}}=\bigotimes_{a \in A} \tau^{A} \circ S_{a}^{-1}
$$

We claim that $\overline{\tau^{A}}$ is ergodic. For that we will show that the $\overline{\tau^{A}}$-skew product extension of $V^{A}$ is ergodic. Take $g \in G, x=\left(x_{b}\right)_{b \in A} \in X^{A}$ and $y=\left(y_{b}\right)_{b \in A} \in K^{A}$. Then $(x, y)=\left(x_{a}, y_{a}\right)_{a \in A} \in(X \times K)^{A}$ and

$$
\begin{aligned}
\left(\left(V^{A}\right)_{g}^{\overline{\tau^{A}}}(x, y)\right)_{a}=\left(V_{g}^{A} x, \overline{\tau^{A}}(g, x)+y\right)_{a} & =\left(V_{g} x_{a}, \tau^{A}\left(g, S_{a}^{-1} x\right)+y_{a}\right) \\
& =\left(V_{g} x_{a}, \tau\left(g, x_{a}\right)+y_{a}\right)=V_{g}^{\tau}\left(x_{a}, y_{a}\right)
\end{aligned}
$$


Thus, $\left(V^{A}\right)^{\overline{\tau^{A}}}=\left(V^{\tau}\right)^{A}$, where $\left(V^{\tau}\right)^{A}:=\bigotimes_{a \in A} V^{\tau}$. Since the skew product $V^{\tau}$ is weakly mixing, $\left(V^{A}\right)^{\overline{\tau^{A}}}$ is ergodic, i.e. $\overline{\tau^{A}}$ is ergodic, as claimed.

Denote by $\mathcal{V}$ the $V^{A}$-orbit equivalence relation on $X^{A}$. It is standard to verify that (iii) implies that $\left\{S_{a} \mid a \in A\right\} \cap[\mathcal{V}]=\{$ Id $\}$. In a similar way, we deduce from the condition of the lemma that $A \cap[\mathcal{R}]=\{\operatorname{Id}\}$. Therefore we may apply the main result from $[\mathrm{BeG}]$ : there is a measure preserving isomorphism $L: X \rightarrow X^{A}$ and a map $A \ni a \mapsto \gamma_{a} \in[\mathcal{V}]$ such that $(L \times L)(\mathcal{R})=\mathcal{V}$ and $L a L^{-1}=\gamma_{a} S_{a}^{-1}$ for all $a \in A$.

We note that $\tau^{A}$ extends naturally to $[\mathcal{V}]$. Indeed, for each $\gamma \in[\mathcal{V}]$, there is a unique $(\bmod 0)$ partition $X^{A}=\bigsqcup_{g \in G} Z_{g}$ of $X^{A}$ into Borel subsets $Z_{g}, g \in G$, such that $\gamma z=\left(V^{A}\right)_{g} z$ whenever $z \in Z_{g}$. We now set $\tau^{A}(\gamma, z):=\tau^{A}(g, z)$ whenever $z \in Z_{g}$. Since $V^{A}$ is free, $\tau^{A}(\gamma,$.$) is well defined. It is easy to verify that$

$$
\tau^{A}\left(\gamma_{1} \gamma_{2}, z\right)=\tau^{A}\left(\gamma_{1}, \gamma_{2} z\right) \tau^{A}\left(\gamma_{2}, z\right)
$$

at a.e. $z$ for all $\gamma_{1}, \gamma_{2} \in[\mathcal{V}]$. We now define a cocycle $\omega$ of $R$ with values in $K$ by setting

$$
\omega(g, x):=\tau^{A}\left(L R_{g} L^{-1}, L x\right) \quad \text { for all } g \in G \text { and } x \in X .
$$

For each $a \in A$, we have

$$
\begin{aligned}
\omega \circ a(g, x) & =\omega(g, a x) \\
& =\tau^{A}\left(L R_{g} L^{-1}, \operatorname{Lax}\right) \\
& =\tau^{A}\left(L R_{g} L^{-1}, \gamma_{a} S_{a}^{-1} L x\right) \\
& =f\left(R_{g} x\right)+\tau^{A}\left(S_{a}^{-1} L R_{g} L^{-1} S_{a}, S_{a}^{-1} L x\right)-f(x),
\end{aligned}
$$

where $f(x):=\tau^{A}\left(\gamma_{a}, S_{a}^{-1} L x\right)$. Thus, $\omega \circ a$ is cohomologous to $\tau^{A} \circ\left(S_{a}^{-1} L\right)=$ $\left(\tau^{A} \circ S_{a}^{-1}\right) \circ L$. This implies that the cocycle $\bar{\omega}$ of $R$, given by

$$
\bar{\omega}(g, x):=\bigotimes_{a \in A} \omega(g, a x)
$$

for all $x \in X, g \in G$, is cohomologous to the cocycle

$$
\left(\bigotimes_{a \in A} \tau^{A} \circ S_{a}^{-1}\right) \circ L=\overline{\tau^{A}} \circ L
$$

Since $\overline{\tau^{A}}$ is ergodic, $\bar{\omega}$ is ergodic too. Thus the first claim of the lemma is proved.

We now show how to deduce the second claim of the lemma from the first one. Given an ergodic action $W$ of $G$, we denote by $\Lambda_{d}(W)$ the discrete spectrum of $W$. Then $\Lambda_{d}(W)$ is a countable subgroup of $\widehat{G}$. It is know that $R^{\bar{\omega}} \times Q$ is ergodic if and only if $\Lambda_{d}\left(R^{\bar{\omega}}\right) \cap \Lambda_{d}(Q)=\{1\}$. Of course, $\Lambda_{d}(R) \subset \Lambda_{d}\left(R^{\bar{\omega}}\right)$. Since $R \times Q$ is ergodic, $\Lambda_{d}(R) \cap \Lambda_{d}(Q)=\{1\}$. Therefore to prove that $R^{\bar{\omega}} \times Q$ is ergodic it suffices to show that $\Lambda_{d}(R)=\Lambda_{d}\left(R^{\bar{\omega}}\right)$. Suppose that there is $\kappa \in \Lambda_{d}\left(R^{\bar{\omega}}\right) \backslash \Lambda_{d}(R)$. Then for some character $\chi \in \widehat{K^{A}}$, the $\mathbb{T}$-valued cocycle $\chi \circ \bar{\omega}$ of $R$ is cohomologous to the cocycle $G \times X \ni(g, x) \mapsto \kappa(g) \in \mathbb{T}$. Since $\widehat{K^{A}}=\bigoplus_{a \in A} \widehat{K}$, there is a finite non-empty subset $A_{0} \subset A$ and a map $A_{0} \ni a \mapsto \chi_{a} \in \widehat{K} \backslash\{1\}$ such that 
$\chi(k)=\prod_{a \in A_{0}} \chi_{a}\left(k_{a}\right)$ for each $k=\left(k_{a}\right)_{a \in A} \in K^{A}$. Since $A$ is infinite, there is $b \in A$ such that $A_{0} \cap\left(b+A_{0}\right)=\emptyset$. For each subset $B \subset A$, we define by $\bar{\omega}_{B}$ the cocycle $\bigotimes_{a \in B} \omega \circ a$ of $R$ with values in $K^{B}$. Since $\bar{\omega}$ is ergodic, $\bar{\omega}_{B}$ is also ergodic. We note that

$$
\bar{\omega}_{A_{0} \cup\left(b+A_{0}\right)}=\bar{\omega}_{A_{0}} \times \bar{\omega}_{b+A_{0}}=\bar{\omega}_{A_{0}} \times \bar{\omega}_{A_{0}} \circ S_{b}
$$

Therefore the $\mathbb{T}^{2}$-valued cocycle

$$
\chi \circ \bar{\omega}_{A_{0}} \times \chi \circ \bar{\omega}_{A_{0}} \circ S_{b}=\chi \circ \bar{\omega} \times \chi \circ \bar{\omega} \circ S_{b}
$$

of $R$ is also ergodic. However the later cocycle is cohomologous to the cocycle

$$
G \times X \ni(g, x) \mapsto(\kappa(g), \kappa(g))
$$

taking values in the diagonal of the torus $\mathbb{T}^{2}$. Hence this cocycle is not ergodic, a contradiction.

\section{ON $H_{3}(\mathbb{Z})$-ODOMETERS}

Let $\Gamma_{1} \supset \Gamma_{2} \supset \cdots$ be a decreasing sequence of lattices (i.e. cofinite subgroups) in $H_{3}(\mathbb{Z})$. Denote by $T=\left(T_{g}\right)_{g \in H_{3}(\mathbb{Z})}$ the associated $H_{3}(\mathbb{Z})$-odometer. Let $(X, \mu)$ be the space of this odometer. We call $T$ normal if $\Gamma_{j}$ is normal in $H_{3}(\mathbb{Z})$ for each $j .^{26}$

If $T$ is normal then $X$ is a compact totally disconnected group and $\mu$ is the normalized Haar measure on $X$. Indeed, we obtain a sequence

$$
H_{3}(\mathbb{Z}) / \Gamma_{1} \leftarrow H_{3}(\mathbb{Z}) / \Gamma_{2} \leftarrow \cdots
$$

of finite groups $H_{3}(\mathbb{Z}) / \Gamma_{j}$ and canonical onto homomorphisms such that $X=$ proj $\lim _{j \rightarrow \infty} H_{3}(\mathbb{Z}) / \Gamma_{j}$. Moreover, a group homomorphism $\varphi: H_{3}(\mathbb{Z}) \rightarrow X$ is well defined by the formula $\varphi(g)=\left(\varphi(g)_{j}\right)_{j=1}^{\infty}$, where $\varphi(g)_{j}:=g \Gamma_{j}$. Of course, $\varphi\left(H_{3}(\mathbb{Z})\right)$ is dense in $X$. It is easy to see that $T_{g} x=\varphi(g) x$ for all $g \in H_{3}(\mathbb{Z})$ and $x \in X$. Hence $T$ has a pure point spectrum in the sense of [Ma3]. Moreover, $T$ is normal in the sense of [Zi]. Hence [Fa, Corollary 2] yields the following.

Corollary 8.1. The normal $H_{3}(\mathbb{Z})$-odometers are isospectral.

We also note that every ergodic 2 -fold self-joining of a normal $H_{3}(\mathbb{Z})$-odometer $T$ is off-diagonal. Hence $T$ is 2 -fold simple [dJRu].

Let $L_{j}$ denote the left regular representation of $H_{3}(\mathbb{R}) / \Gamma_{j}$. Let $\mathcal{I}_{j}$ stand for the unitary dual of $H_{3}(\mathbb{R}) / \Gamma_{j} \cdot{ }^{27}$ It is well known that (up to the unitary equivalence) $L_{j}=\bigoplus_{\tau \in I_{j}} \bigoplus_{1}^{d_{\tau}} \tau$, where $d_{\tau}$ is the dimension of $\tau$. In particular, \# $\left(H_{3}(\mathbb{R}) / \Gamma_{j}\right)=$

\footnotetext{
${ }^{26}$ There is a difference in nomenclature used in our paper and [ $\left.\mathrm{Li}-\mathrm{Ug}\right]$. By an $H_{3}(\mathbb{Z})$-odometer the authors of $[\mathrm{Li}-\mathrm{Ug}]$ mean a free normal $H_{3}(\mathbb{Z})$-odometer. An $H_{3}(\mathbb{Z})$-odometer satisfying the condition $\bigcap_{j=1}^{\infty} \Gamma_{n}=\{1\}$ is called a $H_{3}(\mathbb{Z})$-subodometer there. The $H_{3}(\mathbb{Z})$-odometers which do not satisfy this condition were not considered there. We prefer to use the term "normal" to make it consistent with the well known normality concept introduced by R. Zimmer in [Zi] (see also $[\mathrm{Fa}])$.

${ }^{27}$ Since we do not distinguish between unitarily equivalent representation, we consider elements of $\mathcal{I}_{j}$ as irreducible unitary representations of $H_{3}(\mathbb{R}) / \Gamma_{j}$ rather then their unitary equivalence classes.
} 
$\sum_{\tau \in \mathcal{I}_{j}} d_{\tau}^{2}$. Moreover, $\# \mathcal{I}_{j}$ equals the cardinality of the set of congugacy classes in $H_{3}(\mathbb{R}) / \Gamma_{j}$. Denote by $U_{T}$ the Koopman unitary representation of $H_{3}(\mathbb{Z})$ generated by $T$. The canonical projection $X \rightarrow H_{3}(\mathbb{R}) / \Gamma_{j}$ generates an embedding $L^{2}\left(H_{3}(\mathbb{R}) / \Gamma_{j}\right) \subset X$. Therefore we obtain an increasing sequence

$$
L^{2}\left(H_{3}(\mathbb{R}) / \Gamma_{1}\right) \subset L^{2}\left(H_{3}(\mathbb{R}) / \Gamma_{2}\right) \subset \cdots
$$

of $U_{T}$-invariant subspaces whose union is dense in $L^{2}(X)$ and such that the restriction $U_{T}\left\lceil L^{2}\left(H_{3}(\mathbb{R}) / \Gamma_{j}\right)\right.$ is unitarily equivalent to $L_{j} \circ p_{j}$, where $p_{j}: H_{3}(\mathbb{R}) \rightarrow$ $H_{3}(\mathbb{R}) / \Gamma_{j}$ is the canonical projection. This implies that

$$
\left\{\tau \circ p_{1} \mid \tau \in \mathcal{I}_{1}\right\} \subset\left\{\tau \circ p_{2} \mid \tau \in \mathcal{I}_{2}\right\} \subset \cdots
$$

and we obtain the following decomposition of $U_{T}$ into the orthogonal sum of irreducible finite dimensional unitary representations of $H_{3}(\mathbb{Z})$.

Theorem 8.2. Let $\mathcal{I}_{T}:=\bigcup_{j \in \mathbb{N}}\left\{\tau \circ p_{j} \mid \tau \in \mathcal{I}_{j}\right\}$ and $d_{\iota}$ is the dimension of $\iota$. Then we have

$$
U_{T}=\bigoplus_{\iota \in \mathcal{I}_{T}} \bigoplus_{1}^{d_{\iota}} \iota
$$

An explicit computation of $\mathcal{I}_{T}$ in terms of the sequence $\left(\Gamma_{j}\right)_{j=1}^{\infty}$ was done in [Li-Ug].

Corollary 8.3. Two normal $H_{3}(\mathbb{Z})$-odometers $T$ and $R$ are (measure theoretically) isomorphic if and only if $\mathcal{I}_{T}=\mathcal{I}_{R}$.

We now provide an example of non-isomorphic normal $H_{3}(\mathbb{Z})$-odometers $V$ and $V^{\prime}$ such that the Koopman representations of $H_{3}(\mathbb{R})$ generated by the $H_{3}(\mathbb{R})$ odometers associated with the same sequences of lattices (as $V$ and $V^{\prime}$ respectively) are unitarily equivalent. ${ }^{28}$ We proceed it with a lemma which was utilized in $[\mathrm{Li}-\mathrm{Ug}]$ with reference to $[\mathrm{CoPe}]$. We provide an alternative proof.

Lemma 8.4. Let $V$ be the $H_{3}(\mathbb{Z})$-odometer associated with a sequence $\left(\Gamma_{j}\right)_{j=1}^{\infty}$ of normal lattices in $H_{3}(\mathbb{Z})$ such that $\bigcap_{j=1}^{\infty} \Gamma_{j}=\{1\}$. Let $\Sigma$ be a lattice in $H_{3}(\mathbb{Z})$ such that the homogeneous space $H_{3}(\mathbb{Z}) / \Sigma$ is a factor, say $\mathfrak{F}$, of $V$. Then there is $j_{0}>0$ such that $\Sigma \supset \Gamma_{j_{0}}$.

Proof. Since $V$ is 2-fold simple, we apply the Veech theorem [dJRu]: there is a compact subgroup $K$ in the centralizer $C(V)$ of $V$ such that $\mathfrak{F}$ is the $\sigma$-algebra Fix $K$ of subsets fixed by $K$. Since $\mathfrak{F}$ is finite, $K$ is open. We recall that the space of $V$ is a compact group $X=\operatorname{proj} \lim _{j \rightarrow \infty} H_{3}(\mathbb{Z}) / \Gamma_{j}$. Hence the centralizer $C(V)$ is isomorphic to $X$ acting on itself by right translations. ${ }^{29}$ Let $\Lambda_{j}$ be the kernel of the canonical projection $q_{j}: X \rightarrow H_{3}(\mathbb{Z}) / \Gamma_{j}$. Then $\Lambda_{j}$ is on open subgroup in $X$, $\Lambda_{1} \supset \Lambda_{2} \supset \cdots$ and $\bigcap_{j=1}^{\infty} \Lambda_{j}=\{1\}$. Therefore there is $j_{0}>0$ such that $K \supset \Lambda_{j_{0}}$. Hence $\mathfrak{F} \subset$ Fix $\Lambda_{j_{0}}$. However Fix $\Lambda_{j_{0}}$ is exactly the factor of $V$ determined by $q_{j_{0}}$. Thus we obtain that $H_{3}(\mathbb{Z}) / \Sigma$ is a factor of $H_{3}(\mathbb{Z}) / \Gamma_{j_{0}}$. Since $\Gamma_{j_{0}}$ is normal in $H_{3}(\mathbb{Z})$, it follows that $\Sigma \supset \Gamma_{j_{0}}$.

\footnotetext{
${ }^{28}$ We note that the Koopman representations of $H_{3}(\mathbb{Z})$ generated by $V$ and $V^{\prime}$ are not unitarily equivalent by Corollary 8.1. Thus we obtain an example of unitarily non-equivalent Koopman unitary representations $U_{V}$ and $U_{V^{\prime}}$ of $H_{3}(\mathbb{Z})$ with pure point spectrum such that the induced unitary representations $\operatorname{Ind}_{H_{3}(\mathbb{Z})}^{H_{3}(\mathbb{R})}(V)$ and $\operatorname{Ind}_{H_{3}(\mathbb{Z})}^{H_{3}(\mathbb{R})}\left(V^{\prime}\right)$ of $H_{3}(\mathbb{R})$ are unitarily equivalent.

${ }^{29}$ We recall that $V$ acts on $X$ by left translations.
} 
Example 8.5 (cf. [Li-Ug, Example 4.9]). Let $\left(\Gamma_{n}\right)_{n=1}^{\infty}$ and $\left(\Gamma_{n}^{\prime}\right)_{n=1}^{\infty}$ be as in Example 5.11. Denote by $V$ and $V^{\prime}$ the $H_{3}(\mathbb{Z})$-odometers associated with the sequences $\left(\Gamma_{n}\right)_{n=1}^{\infty}$ and $\left(\Gamma_{n}^{\prime}\right)_{n=1}^{\infty}$ respectively. They are normal. It was shown in $[\mathrm{Li}-\mathrm{Ug}$, Example 4.9] that $T$ and $T^{\prime}$ are not isomorphic. ${ }^{30}$ Indeed, otherwise in view of Lemma 8.4, there is $n>0$ such that $\Gamma_{n}^{\prime} \subset \Gamma_{2}$. However we see that $c(1) a\left(k_{n}\right) \in \Gamma_{n}^{\prime}$ but $c(1) a\left(k_{n}\right) \notin \Gamma_{2}$, a contradiction. On the other hand, $b\left(1 / k_{n}\right) \Gamma_{n}^{\prime} b\left(-1 / k_{n}\right)=\Gamma_{n}$ for each $n \in \mathbb{N}$. Hence the $H_{3}(\mathbb{R})$-odometers $T$ and $T^{\prime}$ associated with $\left(\Gamma_{n}\right)_{n=1}^{\infty}$ and $\left(\Gamma_{n}^{\prime}\right)_{n=1}^{\infty}$ are f-isomorpfic. By Corollary 5.8, the Koopman unitary representations of $H_{3}(\mathbb{R})$ generated by them are unitarily equivalent. We also examine the "symmetry" property for $V$ and $V^{\prime}$. Since $\theta\left(H_{3}(\mathbb{Z})\right)=H_{3}(\mathbb{Z})$, the symmetric $H_{3}(\mathbb{Z})$-actions are defined in a similar way as for the $H_{3}(\mathbb{R})$-actions. It is easy to see that $V$ is symmetric. It is straightforward to verify that

$$
\theta\left(\Gamma_{n}^{\prime}\right)=a\left(-1 / k_{n}\right) \Gamma_{n} a\left(1 / k_{n}\right)=\left\{c\left(k_{n} j_{3}+j_{2}\right) b\left(k_{n} j_{2}\right) a\left(k_{n} j_{1}\right) \mid j_{1}, j_{2}, j_{3} \in \mathbb{Z}\right\}
$$

for each $n$. If $V^{\prime}$ and $V^{\prime} \circ \theta$ were isomorphic then by Lemma 8.4, there is $n>0$ such that $\theta\left(\Gamma_{n}^{\prime}\right) \subset \Gamma_{2}^{\prime}$. However we see that $c\left(k_{n}+1\right) b\left(k_{n}\right) \in \theta\left(\Gamma_{n}^{\prime}\right)$ but $c\left(k_{n}+1\right) b\left(k_{n}\right) \notin$ $\theta\left(\Gamma_{2}^{\prime}\right)$. This contradiction yields that $V^{\prime}$ is not symmetric.

\section{Concluding Remarks and open PRoblems}

In view of Corollary 8.1, we ask the following natural question.

Problem 9.1. Whether the non-normal $H_{3}(\mathbb{Z})$-odometers are isospectral?

Let $G$ be a locally compact second countable group and let $\Gamma$ be a lattice in $G$.

Definition 9.2. We call two probability preserving actions $V$ and $V^{\prime}$ of $\Gamma$ flow equivalent if the induced $G$-actions $\operatorname{Ind}_{\Gamma}^{G}(V)$ and $\operatorname{Ind}_{\Gamma}^{G}\left(V^{\prime}\right)$ are isomorphic.

In the case $G=\mathbb{R}$ and $\Gamma=\mathbb{Z}$ this definition corresponds to the classical concept of flow equivalence in topological dynamics. As was observed, e.g. in [DaLe, Proposition 1.3], if $G$ is Abelian then two ergodic actions of $\Gamma$ are flow equivalent if and only if they are isomorphic.

Problem 9.3. Whether two flow equivalent ergodic actions of $H_{3}(\mathbb{Z})$ are isomorphic? Whether two flow equivalent $H_{3}(\mathbb{Z})$-odometers are isomorphic?

In this connection we make the following remark.

Remark 9.4. Let $V$ and $V^{\prime}$ be two ergodic probability preserving actions of $H_{3}(\mathbb{Z})$. Suppose that they are flow equivalent. Let $(Z, \kappa)$ be the space of $V=\left(V_{\gamma}\right)_{\gamma \in H_{3}(\mathbb{Z})}$

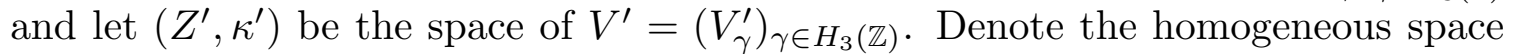
$H_{3}(\mathbb{R}) / H_{3}(\mathbb{Z})$ by $Y$. The translation of $y \in Y$ by $g \in H_{3}(\mathbb{R})$ is denoted by $g * y$. Let $\lambda$ stand for the Haar measure on $Y$. Then $(Y \times Z, \lambda \times \kappa)$ is the space of $\operatorname{Ind}_{H_{3}(\mathbb{Z})}^{H_{3}(\mathbb{R})}(V)$ and $\left(Y \times Z^{\prime}, \lambda \times \kappa^{\prime}\right)$ is the space of $\operatorname{Ind}_{H_{3}(\mathbb{Z})}^{H_{3}(\mathbb{R})}\left(V^{\prime}\right)$. Thus $Y$ is a factor of both $V$ and $V^{\prime}$. Let $R$ be a measure preserving isomorphism of $Y \times Z$ onto $Y \times Z^{\prime}$ that conjugates $\operatorname{Ind}_{H_{3}(\mathbb{Z})}^{H_{3}(\mathbb{R})}(V)$ with $\operatorname{Ind}_{H_{3}(\mathbb{R})}^{H_{3}(\mathbb{R})}\left(V^{\prime}\right)$. Suppose, in addition, that

$$
R \text { maps the factor } Y \text { of } V \text { to the factor } Y \text { of } V^{\prime} \text {. }
$$

\footnotetext{
${ }^{30}$ This fact follows also from Example 5.11 because if $V$ and $V^{\prime}$ are isomorphic then the induced $H_{3}(\mathbb{R})$-actions are also isomorphic. However these induced actions are the $H_{3}(\mathbb{R})$-odometers associated with $\left(\Gamma_{n}\right)_{n=1}^{\infty}$ and $\left(\Gamma_{n}^{\prime}\right)_{n=1}^{\infty}$ respectively. As was shown in Example 5.11, these odometers are not isomorphic.
} 
Then there exists a measurable field $Y \ni y \mapsto R_{y}$ of isomorphisms $R_{y}$ from $(Z, \kappa)$ to $\left(Z^{\prime}, \kappa^{\prime}\right)$ and a transformation $Q$ of $Y$ commuting with the $H_{3}(\mathbb{R})$-action on $Y$ such that $R(y, z)=\left(Q y, R_{y} z\right)$ for a.a. $(y, z) \in Y \times Z$. By [Da2, Lemma 2.1], $Q$ is a translation by an element of the normalizer of $H_{3}(\mathbb{Z})$ in $H_{3}(\mathbb{R})$. A straightforward verification shows that this normalizer is $\{c(t) b(m) a(n) \mid t \in \mathbb{R}, n, m \in \mathbb{Z}\}$. Thus there is $t_{0} \in \mathbb{R}$ such that $Q y=c\left(t_{0}\right) * y$ for $\lambda$-a.a. $y \in Y$. Since $R$ conjugates the induced $H_{3}(\mathbb{R})$-actions, we obtain that

$$
\left(c\left(t_{0}\right) g * y, R_{g * y} V_{h_{s}(g, y)} z\right)=\left(g c\left(t_{0}\right) * y, V_{h_{s}\left(g, c\left(t_{0}\right) * y\right)}^{\prime} R_{y} z\right)
$$

for $(\lambda \times \kappa)$-a.a. $(y, z) \in Y \times Z$ for each $g \in H_{3}(\mathbb{R})$. Here $h_{s}: H_{3}(\mathbb{R}) \times Y \rightarrow H_{3}(\mathbb{Z})$ is the choice cocycle corresponding to a Borel cross-section $s: Y \rightarrow H_{3}(\mathbb{R})$. By Fubini's theorem, there is $y_{0} \in Y$ such that

$$
R_{y_{0}} V_{h_{s}\left(g, y_{0}\right)} z=V_{h_{s}\left(g, c\left(t_{0}\right) * y_{0}\right)}^{\prime} R_{y_{0}} z
$$

for $\kappa$-a.a. $z \in Z$ and all $g$ from the stability group $S_{y_{0}}:=\left\{g \in H_{3}(\mathbb{R}) \mid g * y_{0}=y_{0}\right\}$ of $y_{0}$. We used here that $S_{y_{0}}$ is countable (in fact, it is conjugate to $H_{3}(\mathbb{Z})$ ) to guarantee that the " $\kappa$-a.a. $z \in Z$ " does not depend on $g \in S_{y_{0}}$. Next, we have

$$
\begin{aligned}
h_{s}\left(g, c\left(t_{0}\right) * y_{0}\right) & =s\left(g c\left(t_{0}\right) * y_{0}\right)^{-1} g s\left(c\left(t_{0}\right) * y_{0}\right) \\
& =s\left(c\left(t_{0}\right) * y_{0}\right)^{-1} g s\left(c\left(t_{0}\right) * y_{0}\right) \\
& =r_{0}^{-1} s\left(y_{0}\right)^{-1} g s\left(y_{0}\right) r_{0} \\
& =r_{0}^{-1} h_{s}\left(g, y_{0}\right) r_{0}
\end{aligned}
$$

for some $r_{0} \in H_{3}(\mathbb{Z})$ and all $g \in S_{y_{0}}$. We used the fact that

$$
s\left(c\left(t_{0}\right) * y_{0}\right) \in c\left(t_{0}\right) s\left(y_{0}\right) H_{3}(\mathbb{Z}) .
$$

Now (9-2) implies that

$$
R_{y_{0}} V_{h_{s}\left(g, y_{0}\right)} R_{y_{0}}^{-1}=V_{r_{0}^{-1} h_{s}\left(g, y_{0}\right) r_{0}}^{\prime}=\left(V_{r_{0}}^{\prime}\right)^{-1} V_{h_{s}\left(g, y_{0}\right)}^{\prime} V_{r_{0}}^{\prime} .
$$

Since $\left\{h_{s}\left(g, y_{0}\right) \mid g \in S_{y_{0}}\right\}=H_{3}(\mathbb{Z})$, it follows that $V$ and $V^{\prime}$ are isomorphic.

Thus Problem 9.3 reduces to the following question:

Problem 9.5. Suppose that $\operatorname{Ind}_{H_{3}(\mathbb{Z})}^{H_{3}(\mathbb{R})}(V)$ and $\operatorname{Ind}_{H_{3}(\mathbb{Z})}^{H_{3}(\mathbb{R})}\left(V^{\prime}\right)$ are isomorphic. Is there an isomorphism satisfying (9-1)?

Let $V$ and $V^{\prime}$ be the $H_{3}(\mathbb{Z})$-odometers associated with sequences $\Gamma_{1} \supset \Gamma_{2} \supset \cdots$ and $\Gamma_{1}^{\prime} \supset \Gamma_{2}^{\prime} \supset \cdots$ of lattices in $H_{3}(\mathbb{Z})$ respectively. Let $T$ and $T^{\prime}$ be the $H_{3}(\mathbb{R})$ odometers associated with the same sequences of lattices (considered as lattices in $H_{3}(\mathbb{R})$ ). It follows from Corollary 2.5 that $V$ and $V^{\prime}$ are flow equivalent if and only if $T$ and $T^{\prime}$ are isomorphic. The concept of f-isomorphism for the $H_{3}(\mathbb{R})$-odometers motivated the following definition.

Definition 9.6. Let $T$ and $T^{\prime}$ be two ergodic actions of a locally compact second countable group $G$ on standard probability spaces $(X, \mathfrak{B}, \mu)$ and $\left(X^{\prime}, \mathfrak{B}^{\prime}, \mu^{\prime}\right)$ respectively. We say that $T$ and $T^{\prime}$ are f-isomorphic if there are increasing sequences $\mathfrak{F}_{1} \subset \mathfrak{F}_{2} \subset \cdots$ and $\mathfrak{F}_{1}^{\prime} \subset \mathfrak{F}_{2}^{\prime} \subset \cdots$ of factors of $T$ and $T^{\prime}$ respectively such that 
$\bigvee_{j=1}^{\infty} \mathfrak{F}_{j}=\mathfrak{B}, \bigvee_{j=1}^{\infty} \mathfrak{F}_{j}^{\prime}=\mathfrak{B}^{\prime}$ and $T \uparrow \mathfrak{F}_{j}$ is isomorphic to $T^{\prime} \uparrow \mathfrak{F}_{j}^{\prime}$ for each $j \in \mathbb{N}$ We say that $T$ and $T^{\prime}$ are $F$-isomorphic if there is a finite sequence $R_{1}, \ldots, R_{n}$ of ergodic $G$-actions such that $R_{1}=T, R_{n}=T^{\prime}$ and $R_{j}$ is f-isomorphic to $R_{j+1}$ for each $j=1, \ldots, n-1$. Of course, f-isomorphism is a symmetric relation on the set of ergodic $G$-actions and F-isomorphism is the smallest equivalence relation majorizing f-isomorphism.

We note that F-isomorphism is weaker than isomorphism.

Example 9.7. Let $T$ be a $G$-action with $\operatorname{MSJ}$ (see $[\mathrm{dJRu}])$. Let $(X, \mathfrak{B}, \mu)$ be the space of $T$. Denote by $T \odot T$ the symmetric factor of the Cartesian product $T \times T$, i.e. the restriction of $T \times T$ to the $\sigma$-albegra of subsets invariant under the involution $(x, y) \mapsto(y, x)$. Then it is easy to verify that the $G$-actions $R:=$ $T \times T \times \cdots$ and $R^{\prime}:=(T \odot T) \times T \times T \times \cdots$ are f-isomorphic. Indeed, let $\mathfrak{F}_{n}:=\mathfrak{B}^{\otimes n} \otimes(\mathfrak{B} \odot \mathfrak{B}) \otimes \mathfrak{N} \otimes \mathfrak{N} \otimes \cdots$ and $\mathfrak{F}_{n}^{\prime}:=(\mathfrak{B} \odot \mathfrak{B}) \otimes \mathfrak{B}^{\otimes n} \otimes \mathfrak{N} \otimes \mathfrak{N} \otimes \cdots$. Then $\left(\mathfrak{F}_{n}\right)_{n=1}^{\infty}$ is an increasing sequence of factors of $R$ with $\bigvee_{n=1}^{\infty} \mathfrak{F}_{n}$ being the entire $\sigma$-algebra of $R ;\left(\mathfrak{F}_{n}^{\prime}\right)_{n=1}^{\infty}$ is an increasing sequence of factors of $R^{\prime}$ with $\bigvee_{n=1}^{\infty} \mathfrak{F}_{n}^{\prime}$ being the entire $\sigma$-algebra of $R^{\prime}$; and $R \uparrow \mathfrak{F}_{n}$ is isomorphic to $R^{\prime} \uparrow \mathfrak{F}_{n}^{\prime}$ for each $n$. On the other hand, $R$ and $R^{\prime}$ are not isomorphic (see [dJRu]).

Problem 9.8.

(1) Give an example of two ergodic $G$-actions which are F-isomorphic but not f-isomorphic.

(2) We note that in Example 9.7 the actions $R$ and $R^{\prime}$ are weakly isomorphic. Are there F-isomorphic $G$-actions which are not weakly isomorphic?

(3) Are there F-isomorphic $G$-actions which are not Markov quasi-equivalent? ${ }^{31}$

\section{REFERENCES}

[AGH] L. Auslander, L. Green and F. Hahn, Flows on homogeneous spaces, Princeton University Press, Princeton, N.J., 1963.

[CRa] J.-P. Conze and A. Raugi, On the ergodic decomposition of a cocycle, Colloq. Math. 117 (2009), 121-156.

[BeG] S. I. Bezuglyi and V. Ya. Golodets, Outer conjugacy of the actions of countable amenable groups on a measure space, Izv. Akad. Nauk SSSR Ser. Mat. 50 (1986), 643-660; Math. USSR-Izv. 29 (1987), 1-18.

[CoPe] M. I. Cortez and S. Petit, G-odometers and their almost 1-1 extensions, J. London Math. Soc. 78 (2008), 1-20.

[Da1] A. I. Danilenko, Comparison of cocycles of measured equivalence relation and lifting problems, Ergod. Th. \& Dyn. Syst. 18 (1998), 125-151.

[Da2] A. I. Danilenko, On simplicity concepts for ergodic actions, J. d'Anal. Math. 102 (2007), 77-117.

[Da3] A. I. Danilenko, $(C, F)$-Actions in Ergodic Theory, Progress in Mathematics, vol. 265, 2008, pp. 325-351.

[Da4] A. I. Danilenko, Mixing actions of Heisenberg group, Ergod. Th. \& Dyn. Syst. (to appear).

[DaLe] A. I. Danilenko and M. Lemańczyk, Spectral multiplicities for ergodic flows, DCDS Series A 33 (2013), 4271-4289.

[dJRu] A. del Junco and D. Rudolph, On ergodic actions whose self-joinings are graphs, Erg. Theory \& Dynam. Sys. 7 (1987), 531-557.

[Fa] R. C. Fabec, Normal ergodic actions and extensions, Isr. J. Math. 40 (1981), 175-186.

[Fe] J. M. G. Fell, A Hausdorff topology for the closed subsets of a locally compact nonHausdorff space, Proc. Amer. Math. Soc. 13 (1962), 472-476.

\footnotetext{
${ }^{31}$ See $[$ FPS] for the definitions.
} 
[FPS] K. Fraczek, A. Piekniewska and D. Skrenty, Mixing automorphisms which are Markov quasi-equivalent but not weakly isomorphic, preprint.

[GoSi] V. Ya. Golodets and S. D. Sinelshchikov, On the conjugacy and isomorphism problems for stabilizers of Lie group actions, Ergodic Theory and Dynamical Systems 19 (1999), 391-411.

[Ho] R. Howe, On Frobenius reciprocity for unipotent algebraic group over Q, Amer. J. Math. 93 (1971), 163-172.

[Ki] A. A. Kirillov, Lectures on the orbit method, Amer. Math. Soc., Providence, R. I., 2004.

[Le-We] M. Lemańczyk, J.-P. Thouvenot and B. Weiss, Relative discrete spectrum and joinings, Monatsh. Math. 137 (2002), 57-75.

[Li-Ug] S. Lightwood, A. Şahin and I. Ugarcovici, The structure and the spectrum of Heisenberg odometers, Proc. Amer. Math. Soc. (to appear).

[Ma1] G. W. Mackey, Induced representations of locally compact groups. I, Ann. Math. 55 (1952), 101-139.

[Ma2] G. W. Mackey, Borel structure in groups and their duals, Trans. Amer. Math. Soc. 85 (1957), 134-165.

[Ma3] G. W. Mackey, Ergodic transformation groups with a pure point spectrum, Illinois J. Math. 8 (1964), 593-600.

[Mo] C. C. Moore, Decomposition of unitary representations defined by discrete subgroups of nilpotent groups, Ann. Math. 82 (1965), 146-182.

[Ne] J. von Neumann, Zur Operatorenmethode in der Klassischen Mechanik, Ann. Math. 33 (1932), 587-642.

[Ra] M. S. Raghunathan, Discrete subgroups of Lie groups, Springer, New York, 1972.

[Ri] L. Richardson, Decomposition of the $L^{2}$-space of a general compact nilmanifolds, Amer. J. Math. 93 (1971), 173-190.

[Sc] K. Schmidt, Cocycles of ergodic transformation groups, Lecture Notes in Mathematics, Vol. 1, MacMillan, India, 1977.

[To] J. A. Todd, On a conjecture in group theory, J. London Math. Soc. 25 (1950), 246.

[Zi] R. J. Zimmer, Extensions of ergodic group actions, Illinois J. Math. 20 (1976), 373-409.

Institute for Low Temperature Physics \& Engineering of National Academy of Sciences of Ukraine, 47 Lenin Ave., Kharkov, 61164, UKRAine

E-mail address: alexandre.danilenko@gmail.com

Faculty of Mathematics and Computer Science, Nicolaus Copernicus University, ul. Chopina 12/18, 87-100 Toruń, Poland

and Institute of Mathematics, Polish Academy of Sciences ul. Śniadeckich 8, 00-950 Warsaw, Poland

E-mail address: mlem@mat.uni.torun.pl 